Portland State University

PDXScholar

Spring 6-2-2017

\title{
An Efficient Pipeline for Assaying Whole-Genome Plastid Variation for Population Genetics and Phylogeography
}

Brendan F. Kohrn

Portland State University

Follow this and additional works at: https://pdxscholar.library.pdx.edu/open_access_etds

Part of the Biology Commons, and the Plant Sciences Commons

Let us know how access to this document benefits you.

Recommended Citation

Kohrn, Brendan F., "An Efficient Pipeline for Assaying Whole-Genome Plastid Variation for Population Genetics and Phylogeography" (2017). Dissertations and Theses. Paper 4007.

https://doi.org/10.15760/etd.5891

This Thesis is brought to you for free and open access. It has been accepted for inclusion in Dissertations and Theses by an authorized administrator of PDXScholar. Please contact us if we can make this document more accessible: pdxscholar@pdx.edu. 
An Efficient Pipeline for Assaying Whole-Genome Plastid Variation for

Population Genetics and Phylogeography

by

Brendan F. Kohrn

A thesis submitted in partial fulfillment of the requirements for the degree of

\section{Master of Science}

in

Biology

Thesis Committee:
Mitch Cruzan, Chair
Sarah Eppley
Rahul Raghavan

Portland State University

2017 
(C) 2017 Brendan F. Kohrn 


\begin{abstract}
Tracking seed dispersal using traditional, direct measurement approaches is difficult and generally underestimates dispersal distances. Variation in chloroplast haplotypes (cpDNA) offers a way to trace past seed dispersal and to make inferences about factors contributing to present patterns of dispersal. Although cpDNA generally has low levels of intraspecific variation, this can be overcome by assaying the whole chloroplast genome. Whole-genome sequencing is more expensive, but resources can be conserved by pooling samples. Unfortunately, haplotype associations among SNPs are lost in pooled samples and treating SNP frequencies as independent estimates of variation provides biased estimates of genetic distance. I have developed an application, CallHap, that uses a least-squares algorithm to evaluate the fit between observed and predicted SNP frequencies from pooled samples based on network topology, thus enabling pooling for chloroplast sequencing for large-scale studies of chloroplast genomic variation. This method was tested using artificially-constructed test networks and pools, and pooled samples of Lasthenia californica (California goldfields) from Whetstone Prairie, in Southern Oregon, USA. In test networks, CallHap reliably recovered network topologies and haplotype frequencies. Overall, the CallHap pipeline allows for the efficient use of resources for estimation of genetic distance for studies using non-recombining, wholegenome haplotypes, such as intra-specific variation in chloroplast, mitochondrial, bacterial, or viral DNA.
\end{abstract}




\section{Dedication}

This thesis is dedicated to my grandfather, Dr. Lawrence Loeb, and to my parents, Corinne and David Kohrn, for helping me through the long educational trajectory that led me to where I am now, and for their continued support as I move into the future. 


\section{Acknowledgments}

I would like to acknowledge Mitch Cruzan, for helping talk me through different ideas for the pipeline and keeping me focused through the long hours of debugging, Pam Thompson, Monica Grasty, Tina Arredondo, Jaime Schwoch, and Elizabeth Hendrickson, for helping me work through issues with the program and suggesting features, Jessica Persinger for her help with de novo assemblies, my parents and grandparents, for helping support me through my thesis research, and PSU Academic and Research Computing, for making sure the servers kept running while I was developing this pipeline.

This thesis was completed with funding from a NSF Macrosystems Biology grant (award number 1340746) 


\section{Table of Contents}

Abstract




\section{List of Tables}

Table 1: Summary of sequencing lane contents ........................................................... 12

Table 2: Summary of sequencing data for Whetstone Prairie L. californica libraries...... 14

Table 3: RSS values and residual statistics (A) on a per-SNP basis, and (B) on a per-

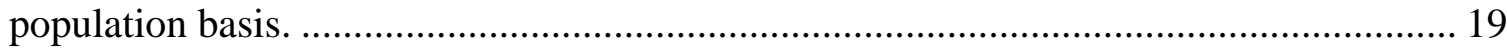




\section{List of Figures}

Fig. 1. SNP frequency contribution from multiple haplotypes where a SNP is shared

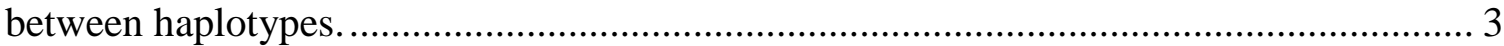

Fig. 2. Haplotype creation and selection of best position in a simple haplotype system.... 7

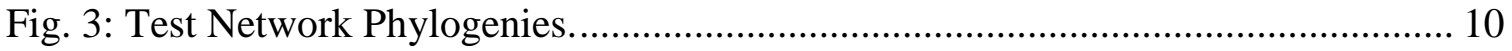

Fig. 4. Resulting phylogeny from one starting condition from Test Network D............. 13

Fig. 5. Haplotypes solution for L. californica de novo alignment. ............................... 18

Fig. 6. Average \% difference between haplotypes within populations vs. between

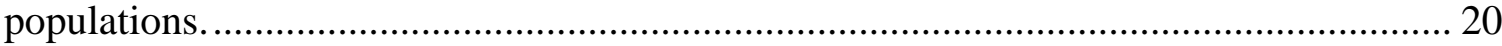

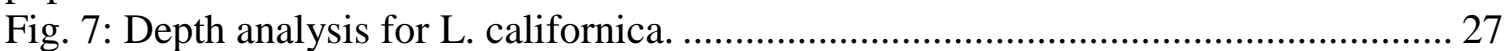




\section{Introduction}

Gene flow includes a number of processes that cause changes in allele frequencies, including movement of gametes (gametophytes) or individuals across the physical landscape (Slatkin, 1987). In the case of plants, movement by gametes is restricted to dispersal of pollen (the male gametophyte) from the location of the paternal individual to the maternal individual, and dispersal of individuals is reduced to movement of seeds. All other life stages of plants are sessile, or have limited mobility through vegetative growth. The distribution of genetic variation within and among plant populations from gene flow can thus be reduced to seed and pollen dispersal. Of these, only seed dispersal has the potential to establish new populations through colonization of vacant sites (Howe and Smallwood, 1982; Nathan and Muller-Landau, 2000).

The movement of seeds to a new site from the location of the maternal parent can occur through a variety of vectors. Some plants have seeds designed to float on the wind (anemochory), while others have seeds which attach themselves to the outside of an animal (ectozoochory), have fleshy fruits meant to be eaten by animals (endozoochory), or just fall off the maternal plant under the influence of gravity (barochory) (Howe and Smallwood, 1982). Traditionally, seed dispersal has been measured by direct observation using a variety of seed trap designs (Gorchov et al., 1993; Kollmann and Goetze, 1998; Godoy and Jordano, 2001), by testing for the presence of seeds in the feces of local herbivorous species (Mouissie et al., 2005), or by observing the movement patterns of dispersal vectors (Kays et al., 2011). Unfortunately, these approaches can be difficult to 
implement and tend to overestimate short-distance seed dispersal while missing longdistance dispersal events (Willson, 1993).

Long-distance seed dispersal is particularly difficult to measure through direct observation approaches, and may be disproportionately important for gene flow and establishing new populations (Cain et al., 2000; Trakhtenbrot et al., 2005). Although measuring seed dispersal is often difficult, genetic markers can be used to track patterns and intensity of historical dispersal. Nuclear genetic markers, including most single nucleotide polymorphisms (SNPs) and microsatellites, offer one potential solution, but variation in these markers within and among populations is affected by both pollen and seed dispersal. In contrast, chloroplast DNA (cpDNA) is inherited maternally in most angiosperms (Corriveau and Coleman, 1988), which means variation in these markers is only affected only by the process of seed dispersal.

Using cpDNA variation (SNPs) to measure genetic distance presents a few challenges. First, chloroplast genomes are non-recombining and effectively haploid (Palmer, 1987), so SNPs common to the same haplotype are inherited together. This feature allows for the reconstruction of network phylogenies that illustrate the relationships among haplotypes, but means that, no matter how many cpDNA SNPs are found, the whole chloroplast can only be treated as a single locus. I found that treating cpDNA SNPs as independent markers will tend to underestimate levels of differentiation and genetic distances among populations, especially when haplotypes share SNPs (Fig. 1). In the past, cpDNA markers have not been considered very useful due to the slow evolutionary rate of chloroplast genomes, which results in low intra-specific variation 
(Palmer, 1987). Modern sequencing methods alleviate this problem by allowing the detection of larger numbers of SNPs across the entire chloroplast genome (Stull et al., 2013). Combinations of SNPs represent chloroplast haplotypes, and are a valuable tool for examining genetic diversity and seed dispersal in angiosperms.

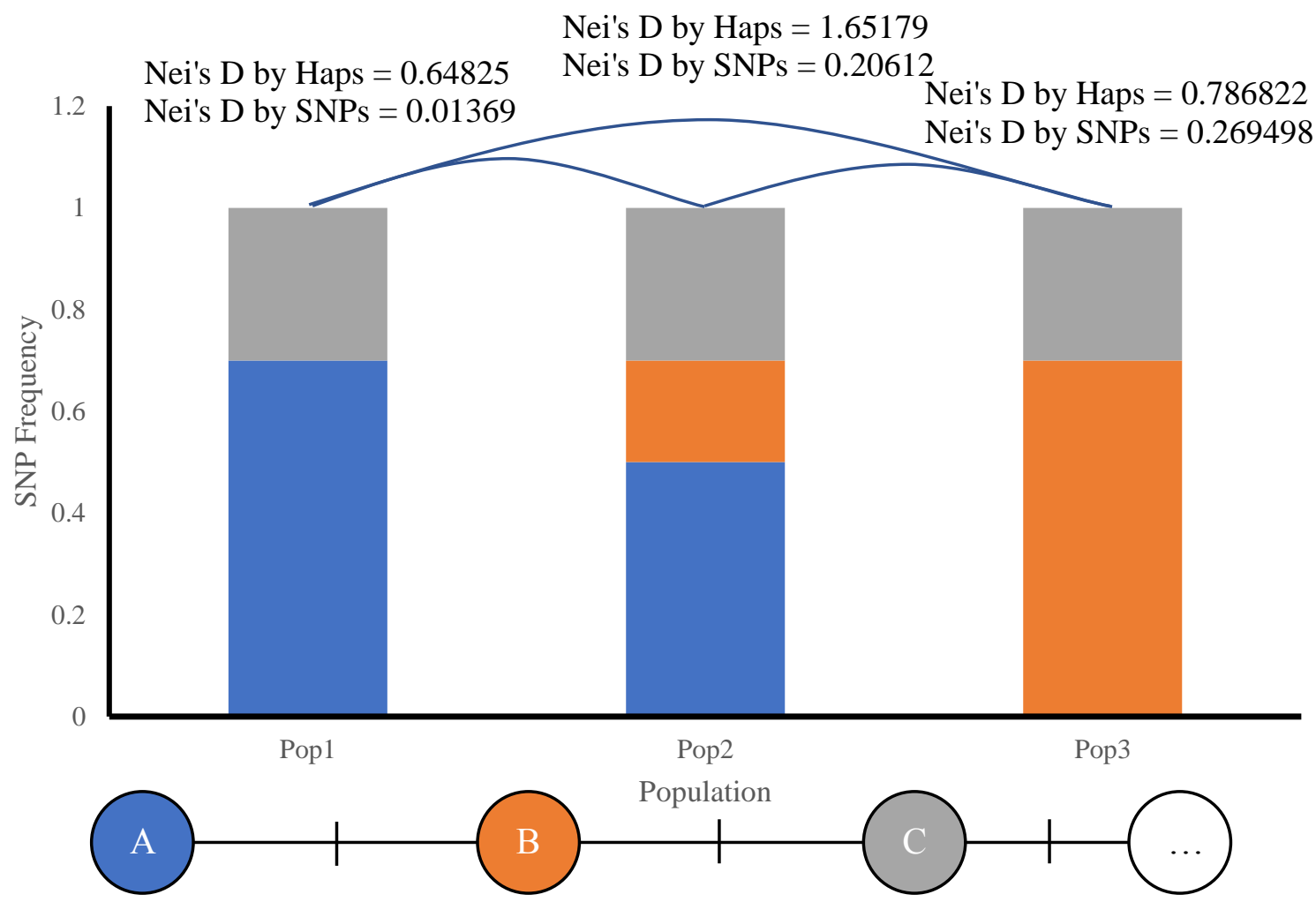

Fig. 1. SNP frequency contribution from multiple haplotypes where a SNP is shared between haplotypes. In this case, each population contains the same three haplotypes, with one being found at a constant frequency in all three populations, while the other two, which share a SNP, are found at varying frequencies in the three populations, such that the overall frequency of that SNP is constant. A network phylogeny showing the three haplotypes and their relatedness to each other is shown below the figure.

When using chloroplast haplotypes for population genetics and phylogeographic studies, cpDNA from many individuals must be sequenced to generate adequate sample sizes for the estimation of genetic parameters. Although sequencing costs have decreased in recent years, sequencing enough samples for a large-scale population genetics study 
still requires significant resource investment (Sboner et al., 2011). Pooling multiple individuals for sequencing has become common as a solution to this problem (Sham et al., 2002; Schlötterer et al., 2014). Unfortunately, pooling cpDNA samples results in the loss of information about the SNP associations that represent each haplotype because DNA sequencing only recovers SNP frequencies (Fig. 1). While there are a number of haplotype reconstruction programs available, these are either aimed exclusively at diploid genomes or at resolving (nuclear) haplotypes over smaller genomic regions (i.e. phasing; Pe'er and Beckmann, 2003; Kirkpatrick et al., 2007; Gasbarra et al., 2011; Kofler et al., 2011). These methods assume some level of recombination, and ultimately are not appropriate for the recovery of haplotypes from the non-recombining chloroplast genome. To solve this problem, I have developed a new bioinformatics pipeline aimed at reducing the cost of population-level surveys of chloroplast diversity by reconstructing chloroplast haplotypes from pooled samples using a sample of sequenced individual chloroplast haplotypes.

Here, I describe sampling and bioinformatics protocols for the examination of haplotype-based population genetics, including the variant filtering and haplotype recovery programs of CallHap. I then test the CallHap haplotype recovery program using a series of artificial networks and pools. Finally, I provide an example of CallHap processing using a set of Lasthenia californica Lindley (Asteraceae) samples collected from Whetstone Prairie, near Medford, OR. 


\section{Bioinformatics Pipeline}

\section{Sample collection and sequencing}

The CallHap pipeline (Appendix A) begins after sampling tissue from some number of individuals (e.g., 20) from each of several populations and extracting DNA from each sampled individual. Equimolar amounts of DNA from each individual are used to make pooled sequencing libraries (PLs). A representative subset of individuals sampled across populations is used to make a collection of single sample libraries (SSLs), which are used to construct a skeleton network phylogeny. Both single and pooled libraries are filtered to concentrate cpDNA using a RNA capture array (Appendix B; Stull et al., 2013), and sequenced using next-generation platforms.

\section{Sequence data processing}

Sequences are processed using cutadapt v1.9.1 (Martin, 2011) for adapter trimming and sickle v1.33 (Joshi and Fass, 2011) for quality trimming. BWA v0.7.5a (Li and Durbin, 2009) is used to align trimmed sequences to a single reference genome. Sequences were sorted using Samtools v0.1.17, read groups were added using Picard Tools v.1.141 (http://broadinstitute.github.io/picard), and indel realignment was carried out using Genome Analysis Toolkit v3.5 (GATK; McKenna et al., 2010). Initial variant calls are made using FreeBayes v1.0.2 (Garrison and Marth, 2012) or other similar programs. 


\section{Variant Filtering}

Variant filtering is carried out using the first of the two major CallHap programs, CallHap_VCF_Filt.py (Program flowchart in Appendix C). This script filters raw variants to ensure that they can be used by the main haplotype caller by removing (a) non-SNP variants, due to the difficulty in calling insertion or deletion type variants (indels) as being in one of two states, (b) variants with low depth or quality, (c) variants that do not have a defined identity across all SSLs and pooled libraries, since the haplotype caller application cannot handle missing values in the matrix of haplotype identities, (e) SNPs in close proximity to indels, due to difficulties in creating correct alignments in these regions. Filters that have a limit (depth filter, indel proximity, and quality filters) are can be modified to meet the demands of a particular study. The variant filter outputs a file containing genotype data for SSLs, a separate file containing SNP frequency data for PLs, and a NEXUS file for network phylogeny creation.

\section{Recovery of haplotypes from pooled samples}

The CallHap Haplotype Caller (CallHap_HapCallr.py, Appendix D) works by iterating through the available SNPs in a pseudo-random order, with SNPs present in SSL (known) haplotypes being processed first. Processing a large number of these random orders increases certainty in haplotype calls. Within each order, an initial estimate of haplotype frequencies is generated using a least squared algorithm (Appendix E) to solve the equation $A x=b$, where A is the binary matrix of SNP identities in various haplotypes, $\mathrm{x}$ is the unknown vector of haplotype frequencies, and $\mathrm{b}$ is the observed vector of SNP frequencies. An overall average Residuals Sums of Squares (RSS) value 
is computed by averaging RSS values based on each PL. In addition, the total RSS value for each SNP is computed.

Next, the algorithm creates new haplotypes based on each SNP for which there exists a non-zero residual in the initial solution (See Appendix F). If the current SNP is present in the known haplotypes, new haplotype creation only considers creating new haplotypes based on the haplotypes at either end of the network phylogeny branch along which this SNP occurs. Otherwise, the algorithm considers every possible new haplotype (Fig. 2). Average RSS values are computed for each possible haplotype attachment point, and the proposed new haplotype matrix and average RSS values are saved for later filtering. This procedure is repeated for each possible solution for all SNPs. Once all possible solutions have been processed for each SNP, the haplotypes matrices are filtered to only keep those that produced the lowest average RSS value (Fig. 2).
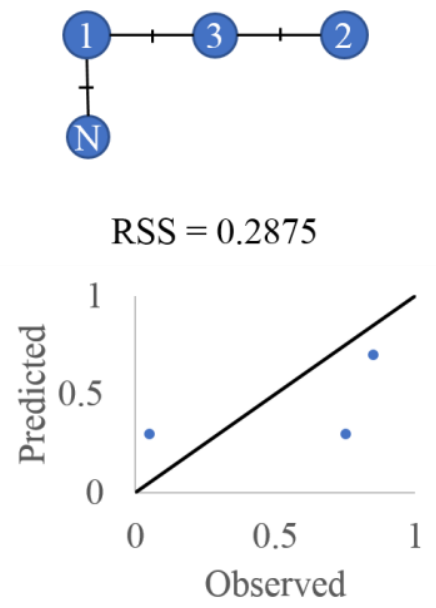

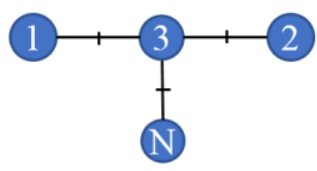

RSS $=0$

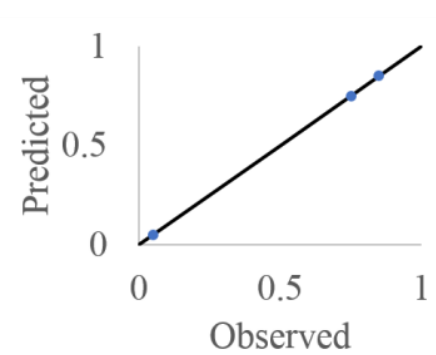

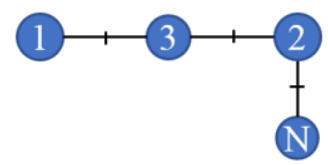

$\mathrm{RSS}=0.1825$

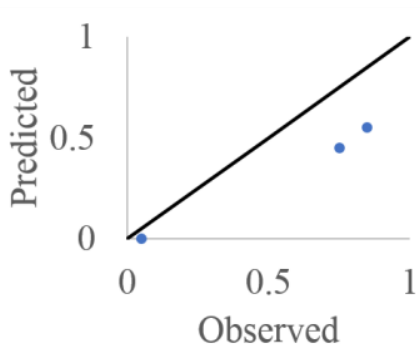

Fig. 2. Haplotype creation and selection of best position in a simple haplotype system. N, in each case, represents the position of the newly-created haplotype. Graphs show predicted vs. observed SNP frequencies. 
Once all SNPs have been processed, the haplotypes matrices are filtered to remove unused haplotypes. Haplotypes matrices are then filtered to keep only those with the lowest Akaike information criterion (AIC; Li et al., 2002). The columns of these matrices (the haplotypes) are taken as binary numbers with 1 representing the reference and 0 the alternate allele, converted into decimal numbers representing the haplotypes, and saved along with the average RSS values produced by the matrices.

After completing all pseudo-random orders, output files are generated showing the raw haplotypes produced in each proposed solution, the percentage of random orderings for which a particular haplotype was produced, the number of times each unique topology was generated and the average RSS value for each, haplotype frequencies in each pool and the RSS value for that pool, VCF files showing predicted SNP frequencies in each pool and RSS for each SNP, a CSV file comparing observed vs. predicted SNP frequencies, and a NEXUS file for network phylogeny creation using PopART (http://popart.otago.ac.nz) or similar. Optionally, a genpop file that can be imported into adegenet (Jombart, 2008) and a STRUCTURE-formatted file (Pritchard et al., 2000; Raj et al., 2014) can also be generated. Haplotype frequencies are presented as number of individuals with that haplotype, and haplotypes are presented as multiple alleles at a single locus (the chloroplast).

After CallHap generates outputs users can examine the resulting topologies and select a final topology based on (1) the average RSS value of the solution, (2) the frequency with which a given topology occurred, and (3) based on the commonality of 
the root haplotype for any mobile new haplotypes not resolved by the first two criteria (Templeton et al., 1992). 


\section{Artificial networks}

Test network phylogenies were created to represent different types of network topologies (Fig. 3). Seven artificial pools containing twenty individuals each were created based on each network, with each pool containing three random haplotypes at frequencies approximating the Poisson distribution. Each set of artificial pools was run through the haplotype caller, using 100 random orders, and 2 iterations per order, with different combinations of "known" haplotypes to see if (a) the correct network topology was recovered by the best solution, and (b), if the correct haplotype frequencies were recovered by the best solution.

A

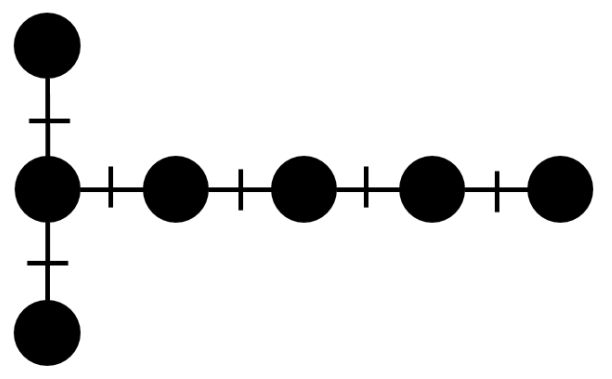

$\mathrm{C}$

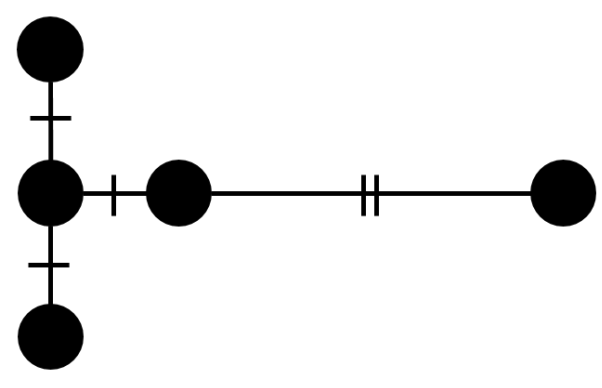

B

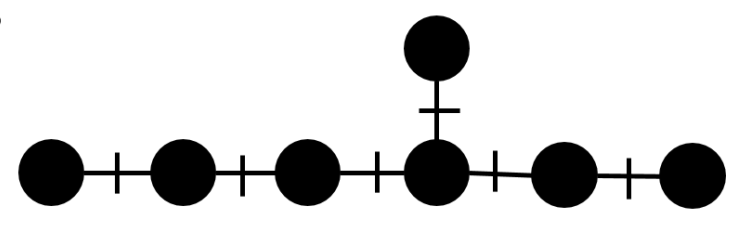

D

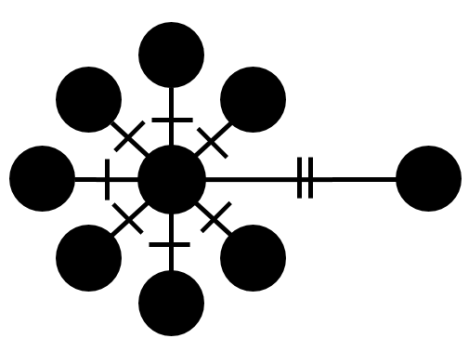

Fig. 3: Test Network Phylogenies. These phylogenies were designed to test the ability of CallHap to recover different topological patterns when starting with different haplotypes; (A) a long branch with every haplotype defined (B) two long branches with all haplotypes defined (C) a long branch with some haplotypes defined, and (D) a cluster with one haplotype further out. 


\section{Testing with Lasthenia californica}

Leaf tissue was collected from 400 individuals across 20 populations of Lasthenia californica located within a 16-hectare area of Whetstone Prairie, near Medford, OR, USA (P. Thompson et. al, unpublished. data). Tissues were dried using silica beads as a desiccant, and DNA was extracted using a Qiagen Plant DNeasy 96 kit (Qiagen, Germantown, MD USA). After DNA extraction, DNA concentration was quantified on a Qubit 3.0 fluorimeter (Thermo Fisher Scientific, Waltham, MA USA), and pooled by population in an equimolar fashion. Library preparation was carried out using a NEBNext Ultra DNA Library Prep Kit (E7370) with NEBNext Multiplex Oligos (E7600; New England Biolabs, Ipswich, MA, USA). Single sample libraries were constructed for at least one individual from each population.

SSLs and PLs were captured using a MYbaits-3 custom cpDNA capture array from MYcroarray (MYcroarray, Ann Arbor, MI, USA; Appendix B). DNA was sequenced on an Illumina HiSeq 2500 Sequencer (Illumina, San Diego, California, USA) using 5 lanes, with 100bp paired-end reads generated for all but six samples, which had 100bp single end reads (Massively Parallel Sequencing Shared Resource Facility, Oregon Health and Science University). The contents of each lane are summarized in Table 1. Sequence alignment was performed both to the published Lasthenia burkei chloroplast genome (Walker et al., 2014) and to an in-house partial de novo reference for $L$. californica (KY965816). SNP calling and variant filtering were performed on both alignments using the pipeline described above with a minimum read depth of 600 and a minimum variant quality of 20. Haplotype calling was performed using information from 
de novo alignments. For the full dataset, haplotype calling was run a second time with any new haplotypes that were consistently added placed in the input haplotypes to help resolve mobile haplotypes.

Table 1: Summary of sequencing lane contents, showing number of Lasthenia californica SSLs and PLs used in analysis on each lane, number of other libraries on each lane, percentage L. californica returns from each lane, and type (single end or paired end) of each run

\begin{tabular}{cccccc} 
Lane & $\begin{array}{c}\text { L. californica } \\
\text { \# SSLs }^{1}\end{array}$ & $\begin{array}{c}\text { L. californica } \\
\text { \# PLs }^{1}\end{array}$ & $\begin{array}{c}\text { Other } \\
\text { Libraries }^{2}\end{array}$ & $\begin{array}{c}\text { \% Returns } \\
\text { L. californica }^{1}\end{array}$ & Run Type \\
\hline 1 & 5 & 0 & 1 & $99.02 \%$ & se \\
2 & 13 & 4 & 7 & $61.39 \%$ & pe \\
3 & 20 & 0 & 28 & $17.53 \%$ & pe \\
4 & 7 & 16 & 31 & $12.42 \%$ & pe \\
5 & 2 & 0 & 52 & $2.14 \%$ & pe
\end{tabular}

${ }^{1}$ Number only reflects libraries used in analysis

${ }^{2}$ These libraries were made using species other than L. californica, or were L. californica libraries unused in this analysis. 


\section{Results}

\section{Test Networks}

Correct haplotype networks were recovered as single lowest RSS value solutions in all starting conditions for three out of four test networks. For the fourth, the correct haplotype network was recovered as the more common of two possible solutions with the lowest RSS value (Fig. 4).

A

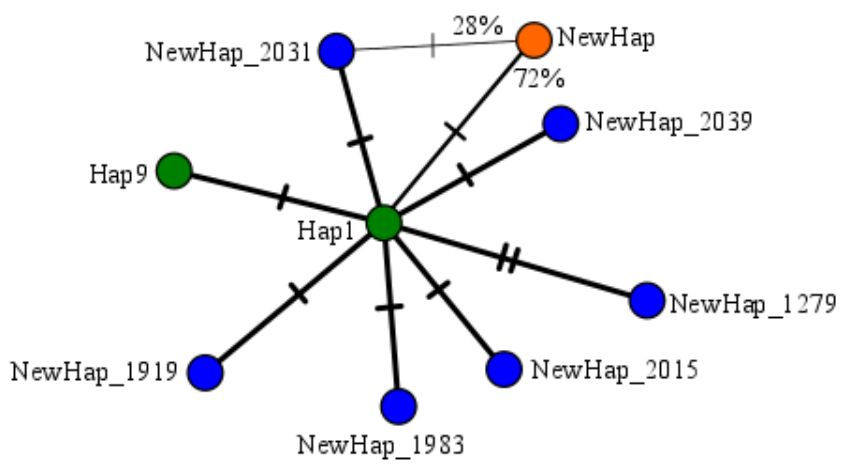

$\mathrm{B}$

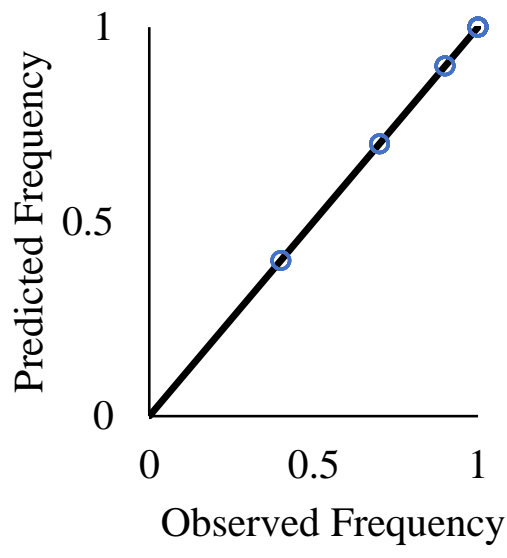

Fig. 4. Resulting phylogeny from one starting condition from Test Network D. (A) Green haplotypes were known at the beginning, blue haplotypes were present in all solutions at the lowest RSS value, and orange haplotypes had ambiguous positions between different solutions. Branch thicknesses are scaled by how many times a solution with the branch occurred, and percentages give exact percent of time a branch occurred. Hash marks indicate number of SNPs along a branch. (B) Regression plot for these solutions. 


\section{Lasthenia californica testing}

Sequencing - Sixty-seven libraries (47 SSLs and 20 PLs) were sequenced, producing a total of $753,355,673$ raw reads. Of these, $88 \%$ of raw reads mapped to the $L$. burkei genome, while $85 \%$ of raw reads mapped to the L. californica de novo genome (Table 2).

Table 2: Summary of sequencing data for Whetstone Prairie L. californica libraries.

\begin{tabular}{|c|c|c|c|c|c|c|}
\hline $\begin{array}{c}\text { Location } \\
\# \\
\end{array}$ & $\begin{array}{c}\text { Individual } \\
\#\end{array}$ & SSL/PL & Type & $\begin{array}{c}\text { Raw } \\
\text { Reads }\end{array}$ & $\begin{array}{l}\text { \% mapped } \\
\text { (L. burkei) }\end{array}$ & $\begin{array}{c}\text { \% mapped } \\
(\text { de novo })\end{array}$ \\
\hline 1 & 5 & SSL & $\mathrm{PE}$ & $2,204,954$ & $86.26 \%$ & $89.11 \%$ \\
\hline 1 & - & PL & PE & $2,838,092$ & $88.45 \%$ & $80.80 \%$ \\
\hline 2 & 20 & SSL & $\mathrm{PE}$ & $3,542,874$ & $87.12 \%$ & $88.29 \%$ \\
\hline 2 & - & PL & $\mathrm{PE}$ & $1,894,746$ & $87.77 \%$ & $81.50 \%$ \\
\hline 3 & 17 & SSL & PE & $11,294,164$ & $86.17 \%$ & $86.89 \%$ \\
\hline 3 & - & PL & PE & $2,046,704$ & $86.31 \%$ & $80.49 \%$ \\
\hline 4 & 5 & SSL & SE & $48,910,678$ & $90.81 \%$ & $88.66 \%$ \\
\hline 4 & - & PL & $\mathrm{PE}$ & $2,101,514$ & $88.19 \%$ & $92.17 \%$ \\
\hline 5 & 8 & SSL & SE & $34,911,454$ & $89.85 \%$ & $86.48 \%$ \\
\hline 5 & - & PL & PE & $1,517,502$ & $85.58 \%$ & $91.55 \%$ \\
\hline 6 & 2 & SSL & SE & $44,544,597$ & $90.29 \%$ & $88.93 \%$ \\
\hline 6 & - & PL & PE & $4,198,354$ & $88.44 \%$ & $91.93 \%$ \\
\hline 7 & 5 & SSL & PE & $7,489,900$ & $86.49 \%$ & $87.94 \%$ \\
\hline 7 & - & PL & PE & $3,589,012$ & $87.38 \%$ & $80.09 \%$ \\
\hline 8 & 2 & SSL & $\mathrm{PE}$ & $2,722,540$ & $89.00 \%$ & $79.70 \%$ \\
\hline 8 & 8 & SSL & $\mathrm{SE}$ & $86,867,128$ & $91.01 \%$ & $89.43 \%$ \\
\hline 8 & 9 & SSL & $\mathrm{PE}$ & $2,348,132$ & $87.79 \%$ & $92.58 \%$ \\
\hline 8 & 12 & SSL & PE & $1,636,222$ & $88.94 \%$ & $88.24 \%$ \\
\hline 8 & 17 & SSL & PE & $1,748,016$ & $89.70 \%$ & $89.38 \%$ \\
\hline 8 & 18 & SSL & PE & $1,398,104$ & $89.73 \%$ & $90.14 \%$ \\
\hline 8 & - & PL & $\mathrm{PE}$ & $10,422,822$ & $86.47 \%$ & $90.17 \%$ \\
\hline 9 & 6 & SSL & SE & $30,468,788$ & $90.13 \%$ & $81.60 \%$ \\
\hline 9 & - & PL & $\mathrm{PE}$ & $7,839,014$ & $87.23 \%$ & $91.86 \%$ \\
\hline 10 & 8 & SSL & PE & $49,460,308$ & $87.26 \%$ & $86.26 \%$ \\
\hline 10 & - & PL & PE & $1,836,746$ & $85.75 \%$ & $80.79 \%$ \\
\hline 11 & 19 & SSL & PE & $6,102,332$ & $89.95 \%$ & $87.85 \%$ \\
\hline 11 & - & PL & PE & $2,141,130$ & $87.33 \%$ & $90.48 \%$ \\
\hline 12 & 4 & SSL & $\mathrm{PE}$ & $2,684,862$ & $90.09 \%$ & $88.83 \%$ \\
\hline 12 & - & PL & $\mathrm{PE}$ & $1,819,176$ & $88.29 \%$ & $90.58 \%$ \\
\hline 13 & 19 & SSL & PE & $20,000,548$ & $83.59 \%$ & $87.93 \%$ \\
\hline
\end{tabular}




\begin{tabular}{|c|c|c|c|c|c|c|}
\hline $\begin{array}{c}\text { Location } \\
\# \\
\end{array}$ & $\begin{array}{c}\text { Individual } \\
\# \\
\end{array}$ & SSL/PL & Type & $\begin{array}{c}\text { Raw } \\
\text { Reads }\end{array}$ & $\begin{array}{l}\text { \% mapped } \\
\text { (L. burkei) }\end{array}$ & $\begin{array}{c}\text { \% mapped } \\
(\text { de novo })\end{array}$ \\
\hline 13 & - & PL & $\mathrm{PE}$ & $1,426,644$ & $87.34 \%$ & $73.74 \%$ \\
\hline 14 & 16 & SSL & $\mathrm{PE}$ & $30,605,932$ & $84.92 \%$ & $90.35 \%$ \\
\hline 14 & - & PL & $\mathrm{PE}$ & $5,877,608$ & $89.81 \%$ & $77.81 \%$ \\
\hline 15 & 1 & SSL & $\mathrm{PE}$ & $3,262,234$ & $89.21 \%$ & $89.69 \%$ \\
\hline 15 & 2 & SSL & $\mathrm{PE}$ & $2,704,746$ & $85.61 \%$ & $86.14 \%$ \\
\hline 15 & 4 & SSL & $\mathrm{PE}$ & $6,493,262$ & $85.65 \%$ & $86.12 \%$ \\
\hline 15 & 5 & SSL & $\mathrm{PE}$ & $4,483,434$ & $89.56 \%$ & $90.09 \%$ \\
\hline 15 & 6 & SSL & $\mathrm{PE}$ & $7,358,804$ & $89.29 \%$ & $89.82 \%$ \\
\hline 15 & 6 & SSL & $\mathrm{PE}$ & $5,371,566$ & $88.69 \%$ & $89.17 \%$ \\
\hline 15 & 7 & SSL & $\mathrm{PE}$ & $5,588,912$ & $88.00 \%$ & $88.35 \%$ \\
\hline 15 & 8 & SSL & $\mathrm{PE}$ & $1,331,344$ & $88.81 \%$ & $89.31 \%$ \\
\hline 15 & 9 & SSL & PE & $2,146,432$ & $87.41 \%$ & $87.94 \%$ \\
\hline 15 & 9 & SSL & $\mathrm{PE}$ & $6,855,194$ & $88.15 \%$ & $88.57 \%$ \\
\hline 15 & 10 & SSL & $\mathrm{PE}$ & $15,648,756$ & $89.90 \%$ & $81.29 \%$ \\
\hline 15 & 10 & SSL & $\mathrm{PE}$ & $8,371,854$ & $87.96 \%$ & $88.32 \%$ \\
\hline 15 & 11 & SSL & $\mathrm{PE}$ & $3,635,032$ & $89.75 \%$ & $90.17 \%$ \\
\hline 15 & 12 & SSL & $\mathrm{PE}$ & $3,030,866$ & $89.05 \%$ & $89.57 \%$ \\
\hline 15 & 13 & SSL & $\mathrm{PE}$ & $2,932,830$ & $89.33 \%$ & $89.79 \%$ \\
\hline 15 & 15 & SSL & PE & $2,565,608$ & $90.12 \%$ & $90.56 \%$ \\
\hline 15 & 16 & SSL & $\mathrm{PE}$ & $7,809,292$ & $88.34 \%$ & $88.99 \%$ \\
\hline 15 & 16 & SSL & $\mathrm{PE}$ & $6,677,584$ & $88.97 \%$ & $89.67 \%$ \\
\hline 15 & 17 & SSL & $\mathrm{PE}$ & $2,207,558$ & $88.91 \%$ & $89.38 \%$ \\
\hline 15 & 18 & SSL & $\mathrm{PE}$ & $7,953,306$ & $89.29 \%$ & $90.09 \%$ \\
\hline 15 & 18 & SSL & $\mathrm{PE}$ & $1,585,198$ & $89.78 \%$ & $90.29 \%$ \\
\hline 15 & 19 & SSL & $\mathrm{PE}$ & $3,384,392$ & $90.23 \%$ & $90.68 \%$ \\
\hline 15 & 20 & SSL & $\mathrm{PE}$ & $6,599,688$ & $90.22 \%$ & $90.67 \%$ \\
\hline 15 & - & PL & $\mathrm{PE}$ & $6,700,776$ & $87.11 \%$ & $81.86 \%$ \\
\hline 16 & 19 & SSL & $\mathrm{PE}$ & $15,407,184$ & $86.15 \%$ & $80.04 \%$ \\
\hline 16 & - & PL & $\mathrm{PE}$ & $1,545,786$ & $86.87 \%$ & $87.50 \%$ \\
\hline 17 & 12 & SSL & $\mathrm{PE}$ & $29,139,552$ & $84.92 \%$ & $75.71 \%$ \\
\hline 17 & - & PL & $\mathrm{PE}$ & $6,241,342$ & $88.75 \%$ & $89.31 \%$ \\
\hline 18 & 2 & SSL & $\mathrm{PE}$ & $40,660,014$ & $86.32 \%$ & $77.87 \%$ \\
\hline 18 & - & PL & $\mathrm{PE}$ & $5,805,872$ & $89.23 \%$ & $89.82 \%$ \\
\hline 19 & 3 & SSL & $\mathrm{PE}$ & $33,083,718$ & $86.01 \%$ & $77.69 \%$ \\
\hline 19 & - & PL & $\mathrm{PE}$ & $6,008,330$ & $88.95 \%$ & $89.47 \%$ \\
\hline 20 & 12 & SSL & $\mathrm{PE}$ & $30,786,938$ & $86.17 \%$ & $78.63 \%$ \\
\hline 20 & - & PL & $\mathrm{PE}$ & $13,827,464$ & $86.09 \%$ & $77.99 \%$ \\
\hline
\end{tabular}




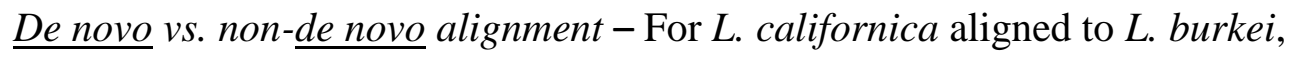
initial variant calling revealed 3154 variants, many of which represented differences between $L$. californica and L. burkei. After variant filtering, 34 SNPs in 16 unique haplotypes were identified across sampled populations of $L$. californica. Comparatively, for L. californica alignment to in house de novo, 978 initial variants were recovered, which simplified to 39 SNPs in 19 unique haplotypes after filtering. Of these, 26 appeared to be identical to SNPs from the $L$. burkei alignment

Initial haplotype calling for the complete $L$. californica data recovered two solutions at a minimum RSS value of 0.003002 , with seven new haplotypes common to all the top three solutions and three unfixed haplotypes. Rerunning with the common haplotypes added to the SSL haplotypes returned a solution with a RSS value of 0.003002 , a solution with a RSS value of 0.003077 , and a solution with a RSS value of 0.003165; these topologies are summarized in Fig. 5, and RSS values are summarized in Table 3. Although the best RSS value solution wasn't the most common solution, the difference in the RSS values was small enough that the solutions are essentially equivalent. Additionally, there were only minor differences in haplotype frequency between the best RSS value solution and the second best RSS value solution. Since the RSS values for the best two solutions were so similar, the more common topology was selected as the best topology.

Average phylogenetic distance was calculated between haplotypes in each pair of populations (Between) or between haplotypes within a single population (Within) using the formula: 


$$
\text { Average } \% \text { difference }_{\mathrm{a}, \mathrm{b}}=\sum_{\mathrm{i}} \sum_{\mathrm{j}} \mathrm{d}_{\mathrm{i}, \mathrm{j}} * \mathrm{p}_{\mathrm{i}, \mathrm{a}} * \mathrm{p}_{\mathrm{j}, \mathrm{b}}
$$

Where $i$ and $j$ are haplotypes, $a$ and $b$ are populations, $p_{i, a}$ is the frequency of haplotype $i$ in population $a$, and $d_{i, j}$ is the number of SNPs different between haplotypes $i$ and $j$. This showed that haplotypes within a population were more similar to each other than haplotypes in different populations (2-sample $\mathrm{t}$-test, $\mathrm{df}=25, \mathrm{t}=6.49, \mathrm{p}<<0.01$; Fig. 6). 


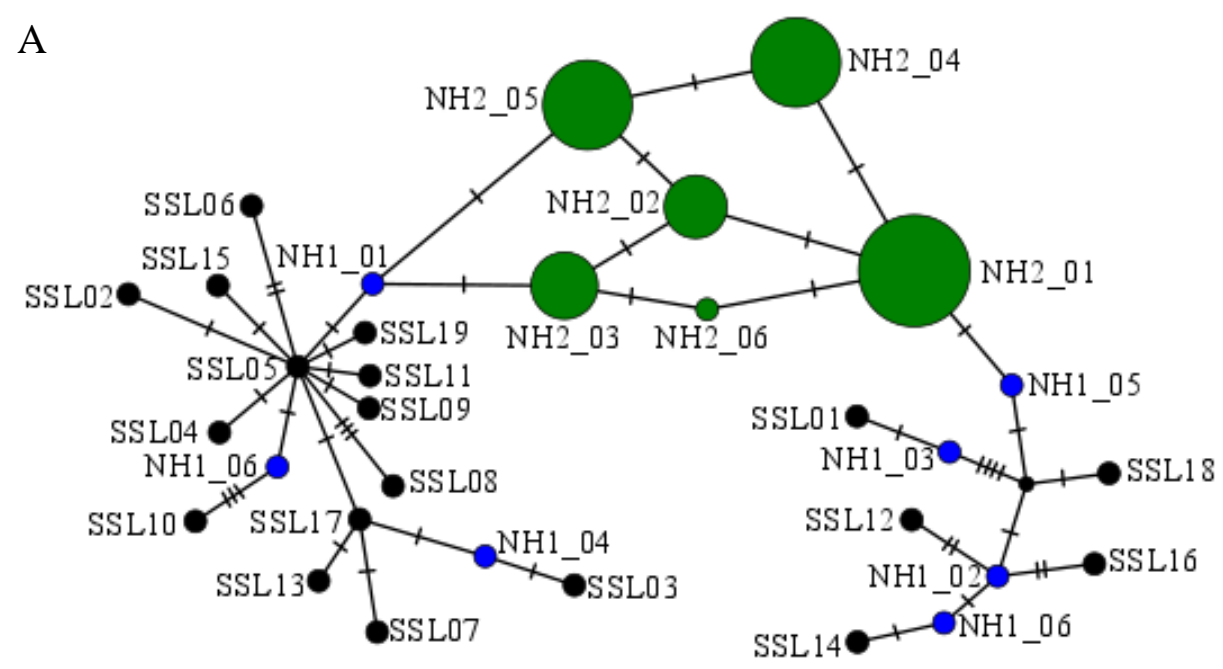

B

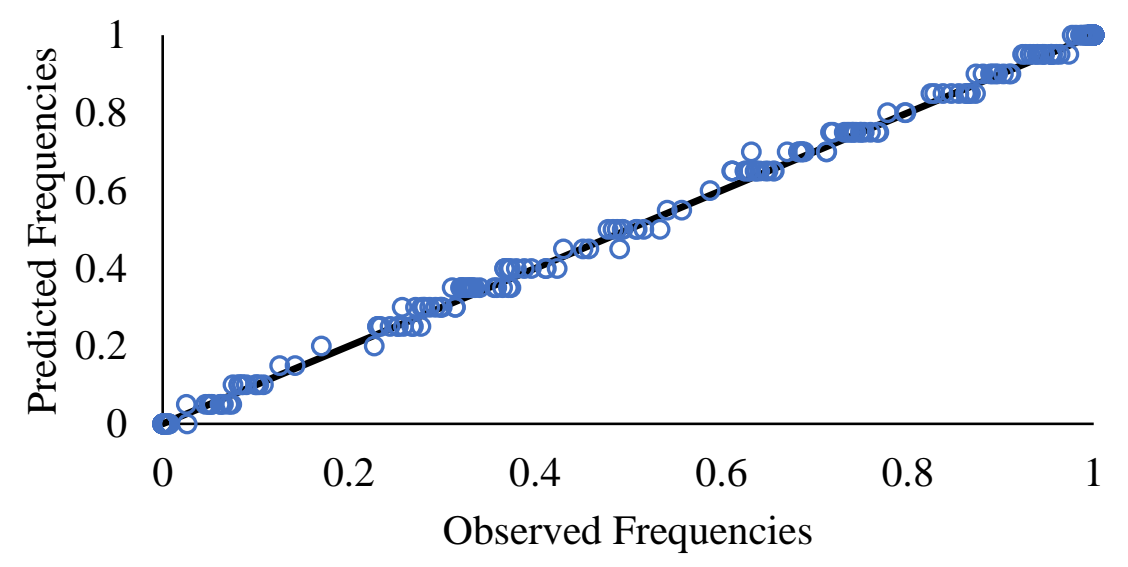

Fig. 5. Haplotypes solution for L. californica de novo alignment. (A) Consolidated network phylogeny for CallHap solutions with the lowest RSS value (0.003002). Black indicates starting haplotypes, blue indicates new haplotypes fixed in the best solutions from the initial haplotype calling run, and green indicates new haplotypes found in the second haplotype calling run. For the second run, node size is scaled to indicate the number of output solutions a new haplotype occurred in. Hash marks indicate number of SNPs along a branch. (B) Regression plot for lowest-RSS value CallHap solutions. 
Table 3: RSS values and residual statistics (A) on a per-SNP basis, and (B) on a per-population basis. A squared residual value of 0.0025 is equivalent to one individual's worth of error.

\begin{tabular}{|c|c|c|c|c|c|}
\hline A ${ }_{\text {SNP \# }}$ & RSS & $\begin{array}{c}\text { Average squared } \\
\text { residual }\end{array}$ & $\begin{array}{l}\text { Standard Deviation } \\
\text { of squared residuals }\end{array}$ & B Population & $\begin{array}{c}\text { RSS } \\
\text { Value } \\
\end{array}$ \\
\hline 0 & 0.000176 & 0.000009 & 0.000032 & 1 & 0.004688 \\
\hline 1 & 0.002585 & 0.000129 & 0.000429 & 2 & 0.000322 \\
\hline 2 & 0.000040 & 0.000002 & 0.000006 & 3 & 0.005565 \\
\hline 3 & 0.000128 & 0.000006 & 0.000026 & 4 & 0.001729 \\
\hline 4 & 0.000071 & 0.000004 & 0.000012 & 5 & 0.005304 \\
\hline 5 & 0.000459 & 0.000023 & 0.000058 & 6 & 0.002121 \\
\hline 6 & 0.001599 & 0.000080 & 0.000154 & 7 & 0.000042 \\
\hline 7 & 0.004566 & 0.000228 & 0.000262 & 8 & 0.003693 \\
\hline 8 & 0.001141 & 0.000057 & 0.000142 & 9 & 0.000446 \\
\hline 9 & 0.006557 & 0.000328 & 0.000486 & 10 & 0.005215 \\
\hline 10 & 0.004619 & 0.000231 & 0.000390 & 11 & 0.004026 \\
\hline 11 & 0.000200 & 0.000010 & 0.000043 & 12 & 0.006501 \\
\hline 12 & 0.000147 & 0.000007 & 0.000032 & 13 & 0.003062 \\
\hline 13 & 0.002009 & 0.000100 & 0.000294 & 14 & 0.004435 \\
\hline 14 & 0.001082 & 0.000054 & 0.000141 & 15 & 0.000325 \\
\hline 15 & 0.000552 & 0.000028 & 0.000107 & 16 & 0.002084 \\
\hline 16 & 0.001887 & 0.000094 & 0.000249 & 17 & 0.000382 \\
\hline 17 & 0.002112 & 0.000106 & 0.000239 & 18 & 0.006086 \\
\hline 18 & 0.000791 & 0.000040 & 0.000099 & 19 & 0.001960 \\
\hline 19 & 0.002005 & 0.000100 & 0.000198 & 20 & 0.003560 \\
\hline 20 & 0.000606 & 0.000030 & 0.000134 & & \\
\hline 21 & 0.000714 & 0.000036 & 0.000119 & & \\
\hline 22 & 0.003955 & 0.000198 & 0.000366 & & \\
\hline 23 & 0.000143 & 0.000007 & 0.000028 & & \\
\hline 24 & 0.000416 & 0.000021 & 0.000090 & & \\
\hline 25 & 0.004510 & 0.000226 & 0.000283 & & \\
\hline 26 & 0.000026 & 0.000001 & 0.000002 & & \\
\hline 27 & 0.010800 & 0.000540 & 0.001008 & & \\
\hline 28 & 0.000448 & 0.000022 & 0.000085 & & \\
\hline 29 & 0.000131 & 0.000007 & 0.000008 & & \\
\hline 30 & 0.001441 & 0.000072 & 0.000318 & & \\
\hline 31 & 0.000947 & 0.000047 & 0.000145 & & \\
\hline 32 & 0.000180 & 0.000009 & 0.000036 & & \\
\hline 33 & 0.000173 & 0.000009 & 0.000024 & & \\
\hline 34 & 0.000744 & 0.000037 & 0.000156 & & \\
\hline 35 & 0.000354 & 0.000018 & 0.000011 & & \\
\hline 36 & 0.000064 & 0.000003 & 0.000008 & & \\
\hline 37 & 0.003147 & 0.000157 & 0.000276 & & \\
\hline 38 & 0.000020 & 0.000001 & 0.000001 & & \\
\hline
\end{tabular}




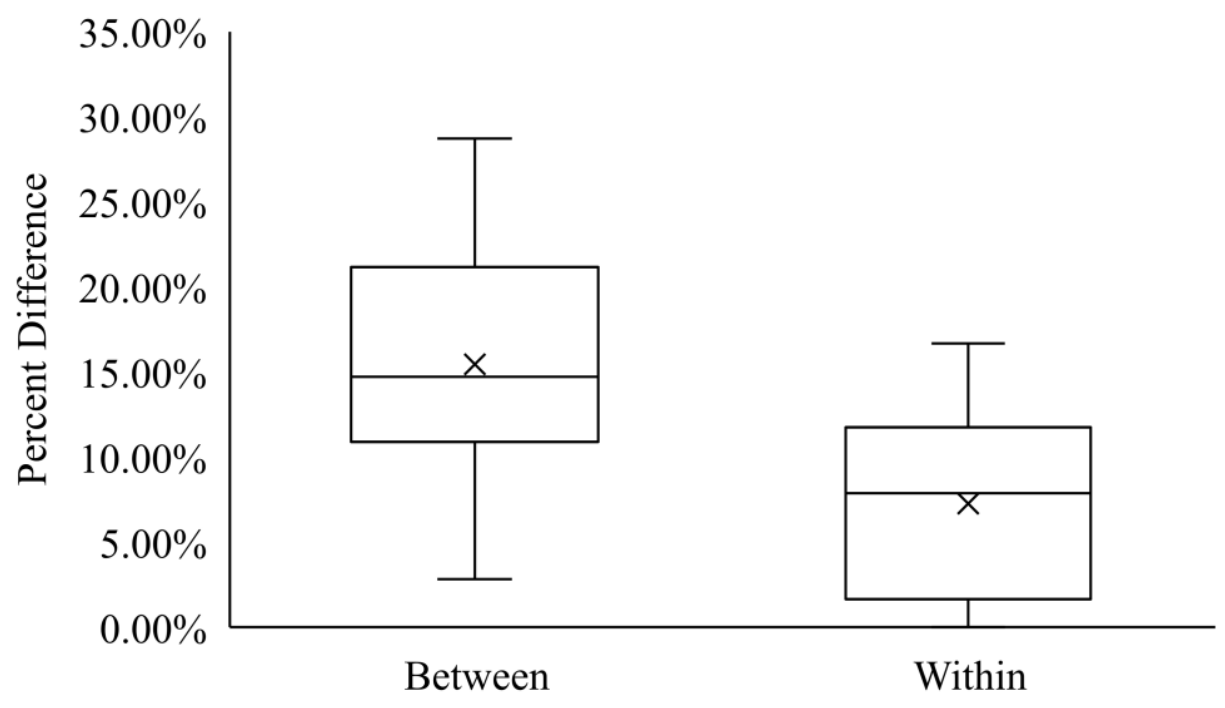

Fig. 6. Average $\%$ difference between haplotypes within populations vs. between populations. 


\section{Discussion}

I have developed a pipeline, CallHap, for efficient examination of cpDNA variation, and tested it using a variety of test networks and a real data set of Lasthenia californica samples from Whetstone Prairie. Here, I present (A): an examination of test network results, (B): considerations for the design of experiments using CallHap, and (C): appropriate protocols for analysis of CallHap outputs. In addition, I provide an explanation for the magnitude of RSS values calculated by CallHap.

\section{Test Networks Results}

Examination of the test network pools shows consistent recovery of haplotype networks from a starting point of two or more haplotypes in the absence of any sequencing error. The presence of two possible solutions in the fourth test network reveals one potential problem that could arise during haplotype construction; if the frequencies for a new haplotype (based on SNPs not present in any of the SSLs) are less than the frequencies for multiple other haplotypes across all PLs, it is possible the new haplotype may be placed ambiguously between multiple locations on the network. When the false haplotype position was not one of the known haplotypes, the correct solution was the more common solution. One solution to this issue would be to add new haplotypes that were found consistently between the solutions with the best RSS values to the starting haplotypes array and rerunning the program. By using the expanded array of haplotypes as a starting point, differences between solutions with the same RSS value may be resolved. Another method involves taking the source DNA samples and creating 
extra PLs by reshuffling the samples in ways that don't reflect the geographic areas the samples were collected in (discussed in more detail later).

Testing also revealed that, with minimal sampling of SSLs, convergence to a best solution was proportional to the centrality of the starting haplotype. As an example, for one of the test pools, all 100 orders converged to the lowest RSS value when the starting haplotype was the most central haplotype, as opposed to 13/100 and 3/100 for starting haplotypes one and two SNPs different from the most central haplotype, respectively. Also, the presence of long branches in the correct topology reduced the frequency with which that topology came up. In cases where CallHap is finding a large number of topologies, rerunning CallHap with a larger number of random orderings, potentially in combination with augmenting the known haplotypes with any haplotypes found universally, may help. In addition, starting with more than one SSL per population will increase the likelihood that the most central haplotype will be included in the SSL haplotypes.

It is apparent from examining the inferred haplotype frequencies for L. californica that RSS values for individual populations differ substantially. There can be many reasons for this; in some cases, high RSS values may be due to a low-quality SNP that was not filtered out correctly. For this reason, even after automated SNP filtering, any remaining SNPs should be visualized using IGV (Thorvaldsdóttir et al., 2013) or other similar programs to ensure quality. Potential issues include SNPs that occur at approximately the same frequency across populations while the other SNPs in the pool 
change frequencies (especially if the major SNP present in the pool changes frequency). In these cases, the inconsistent SNPs are most likely artificial and should be removed.

Another potential cause of high RSS values is a large number of SNPs present at a frequency of more than $1 / n$, where $n$ is the number of individuals in a pool. For example, if a pool contains only three SNPs at such frequencies, an RSS of 0.05 could indicate a problem; for a 20-individual pool, a RSS value of 0.0025 is equivalent to one individualworth of error, so a RSS value of 0.05 under these conditions would indicate an average error of +/- 6.7 individuals for each haplotype present in that pool. If the same RSS value were to occur in a pool where 20 SNPs were present at these frequencies, it would be less of a problem because it would indicate an average error in haplotype frequencies of $+/-1$ individual. In the L. californica data, the average RSS value was 0.003077 , and was less than one individual's worth of error per haplotype present in all of the pools.

\section{Experimental design considerations for CallHap Analyses}

When designing an experiment to feed into the CallHap pipeline, consideration must be given to (1): the spatial scale of sampling, (2): the number of populations sampled, and (3): the size of pooled libraries. In addition, the choice of reference genome for sequence alignment and variant discovery, and the minimum read depth used, is important and needs to be contemplated.

Spatial Scale of Sampling - Experimental designs which produce data for the CallHap pipeline will differ primarily on the geographic scale of sampling. For this purpose, small-scale sampling indicates that populations are sampled at distances smaller than the hypothesized average dispersal distance of the target species, and large-scale 
sampling indicates that populations are sampled at distances greater than the hypothesized average dispersal distance of the target species. At small scales, dispersal is great enough that each haplotype may be found in any location so populations are differentiated primarily by differences in the frequencies of shared haplotypes, meaning that experiments should be designed with one SSL and one PL per population. In this type of experiment there is a lowered likelihood of difficulties in recovering the correct network topology and frequencies.

At large scales, populations in close proximity to each other may represent a unique cluster of related haplotypes, and different sets of haplotypes may occur in separate regions. As shown in the test networks, when only one SSL is available for each cluster, it becomes difficult to place new haplotypes within that cluster. Additionally, if a haplotype is only present in a single population, it is difficult to accurately place the haplotype within the network phylogeny. At large scales, it would be advisable to create artificial pools by pooling DNA from individuals from multiple populations located across the entire range. Notably, these pools should not include the samples used for SSLs, as those haplotypes are already known, and should contain samples at differing concentrations; the purpose of these pools is to help resolve the identity of any new haplotypes inferred by CallHap. Sequencing more than one SSL per population should also be considered in these cases. Sequencing multiple populations per region will also help resolve topologies and haplotype frequencies when the distance between populations within each region occurs at a small scale, and sampled regions occur at a large scale. 
One final complication is that the true scale of a project may not become evident until after starting data analysis. For example, when the L. californica experiment was designed, the hypothesized dispersal range was greater than the distance between populations. However, after sequencing, it turned out that seed dispersal in L. californica much more limited than anticipated. In retrospect, creating artificial pools to help resolve the network topology would have been beneficial.

Pooling and Pooled library size - Many pool-seq protocols pool samples before DNA extraction (Kofler et al., 2012; Martins et al., 2014; Bélanger et al., 2016), but this may generate higher errors in SNP frequencies because equal amounts of tissue may not contain equal amounts of genomic DNA. In contrast, data for use in CallHap comes from libraries where DNA is extracted before being pooled to ensure equimolar proportions of DNA from each individual. While populations of any size could be analyzed, sequencing error, pipet volume, and DNA concentration limit the number of individuals that can be safely placed in a single PL and still give accurate resolution of haplotype frequencies. In addition, as the number of individuals in a PL increases, the frequency that represents a single individual starts to approach the level of error in the sequencing process. On the other hand, if too few individuals per population are used, some haplotypes present in the population may be missed. For example, if 10 individuals per population were used, any haplotype present at a frequency below $10 \%$ would likely go undetected. In the $L$. californica study, a sample size of 20 individuals per population was used; it provided reasonable accuracy in SNP frequency estimates while still capturing a good amount of the haplotype diversity present. More individuals per population could be used by 
sequencing multiple pools per population, processing them as separate populations, and then combining the frequencies after running them through CallHap and before continuing with later population genetics or phylogeographic analysis.

Choosing a Reference Genome - CallHap assumes that SNPs detected by variant calling arise from closely related haplotypes. Because of this, the CallHap pipeline requires that all libraries be aligned to a single reference genome. Since the genome used will have a large influence on the number and quality of SNPs generated, genome selection is an important aspect of any study using CallHap.

In choosing a reference genome to use for CallHap analysis, preference should be given to conspecific references. If no such reference exists, one library of shotgun sequencing should be run; this library can be used to create a de novo reference genome to which the other samples can be aligned. While a de novo can be created using captured cpDNA, the incomplete nature of the capture makes it more difficult to carry out the de novo assembly. If creating a de novo reference is infeasible, it may be possible to obtain limited results using a non-conspecific reference; in this case, the more closelyrelated the reference chloroplast genome is to the study species, the better. Limitations of interspecific references include the addition of artificial SNPs introduced due to alignment ambiguities that may be caused by fixed differences between the chloroplast genomes of the two species.

Minimum Read Depth Selection - Another important parameter is the minimum read depth required to consider a genomic position for analysis. I found that this value changes depending on the peculiarities of different species and sequencing runs; for $L$. 
californica, the optimum read depth was around 600, while for Ranunculus occidentalis Nutt. (Ranunculaceae), the optimum minimum depth was found to be 300-400. To determine the optimum minimum depth, I ran the VCF filter multiple times with different depths, and counted the number of initial unique haplotypes each time. I then selected the optimum depth as the point where the number of haplotypes started to drop off (Fig. 7) or 300, whichever was higher. In general, minimum depth should be no less than $15 *$ the number of individuals in a pool (Sims et al., 2014).

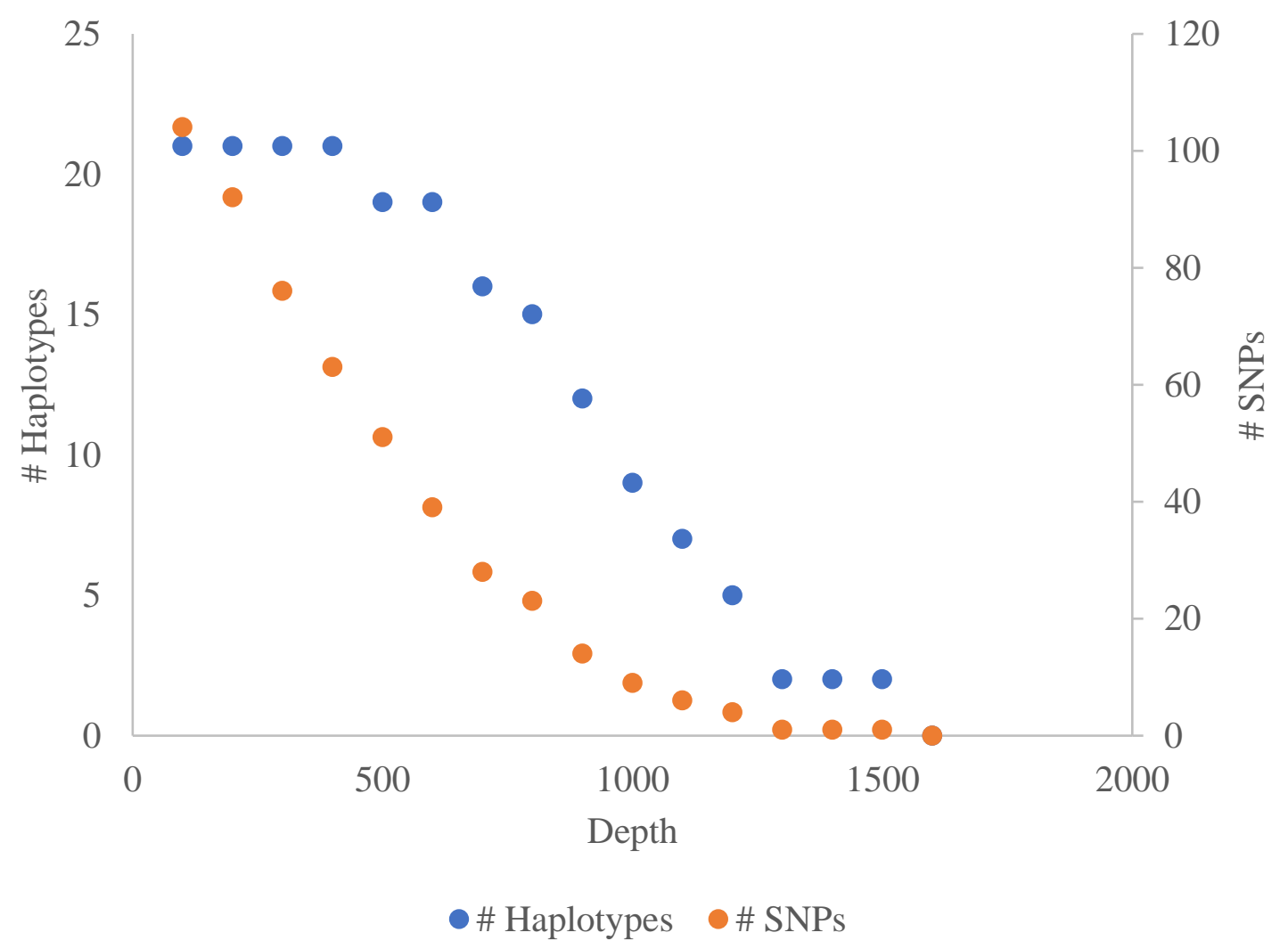

Fig. 7: Depth analysis for L. californica. The number of unique SSL haplotypes starts to drop off at around 600 depth. 


\section{Analysis of CallHap Outputs}

Methods used for analysis of haplotype frequency data from CallHap will vary depending on the goals of the study. Population genetics studies utilizing nuclear genetic markers in diploid organisms typically use Wright's FST (Wright, 1949) or a similar analogue (G $\mathrm{G}_{\mathrm{ST}}$, G'sT, $\mathrm{D}_{\mathrm{ST}}$, etc.; Whitlock, 2011). However, $\mathrm{F}_{\mathrm{ST}}$ is based on comparisons of observed and expected heterozygosity at different scales, and consequently is inappropriate for use with haplotype data. Instead, genetic distance measures that allow for variable ploidies and number of alleles per locus and are not reliant on measures of homo- or heterozygosity—such as Nei's Genetic Distance (Nei’s D; Nei, 1973), Edwards chord distance (Cavalli-Sforza and Edwards, 1967; Edwards, 1971; Hartl et al., 1997), or $\Phi$-statistics (Meirmans, 2006) — and haplotype genetic diversity measures (e.g. unbiased haplotype diversity; Gardner et al., 2015) should be used.

Methods such as Nei's D rely on calculations of the probability that the same combination of alleles will be found in two different populations, and consequently are more appropriate for small-scale studies. When no haplotypes are shared between two populations, Nei's D gives an infinite distance between those populations; such a pattern indicates that dispersal rates among the populations sampled are very low, and that the accumulation of local mutations is the primary factor contributing to the genetic structure of populations. Limited dispersal relative to the scale of sampling will lead to haplotypes within populations being more similar to each other than haplotypes in different populations, as can be seen in the L. californica data. In these cases, phylogeographic methods, the Edwards chord distance, or $\Phi$-statistics will be more appropriate. 
In phylogeographic studies or population genetics studies that are found to be more appropriate for phylogeographic analysis, methods such as Nested Clade Analysis (Templeton, 1998, 2009) or Approximate Bayesian Computation (Csilléry et al., 2010) should be used. These methods explain modern observations with predictions of population history events by comparing observed data to different modeled population histories.

\section{Applications}

The CallHap pipeline has the potential to creating a range of new opportunities for studies of cpDNA population structure, and allows for accurate and economical estimates of seed-mediated gene flow by allowing for the use of pooled population sequencing data for cpDNA and other haploid genetic material. Data for use in the CallHap pipeline comes from population-level sampling of haploid genomes, including plant chloroplast genomes (as presented in this paper), mitochondrial genomes, and prokaryotic bacterial genomes. Because CallHap assumes all generated haplotypes are closely related and requires that all libraries examined be aligned to a single reference genome; this protocol should not be used for microbiome and microbial community studies. Outputs generated by CallHap can be analyzed using a variety of methods, including Nei's genetic distance, Edwards chord distance, $\Phi$-statistics, and a variety of phylogeographic analysis methods including Nested Clade Analysis and Approximate Bayesian Computation.

CallHap is available at https://github.com/cruzan-lab/CallHap. 


\section{References}

Bélanger, S., P. Esteves, I. Clermont, M. Jean, and F. Belzile. 2016. Genotypingby-sequencing on pooled samples and its use in measuring segregation bias during the course of androgenesis in barley. The Plant Genome 9: 0. Available at: https://dl.sciencesocieties.org/publications/tpg/abstracts/9/1/plantgenome2014.10.00 73 [Accessed March 23, 2017].

Cain, M.L., B.G. Milligan, and A.E. StRAnD. 2000. Long-distance seed dispersal in plant populations. American Journal of Botany 87: 1217-1227.

Cavalli-SforZA, L.L., and A W.F. Edwards. 1967. Phylogenetic analysis. Models and estimation procedures. The American Journal of Human Genetics 19: 233-257. Available at: http://www.pubmedcentral.nih.gov/articlerender.fcgi?artid=1706274\&tool=pmcentr ez\&rendertype $=$ abstract.

Corriveau, J.L., and A.W. Coleman. 1988. Rapid Screening Method to Detect Potential Biparental Inheritance of Plastid DNA and Results for Over 200 Angiosperm Species. American Journal of Botany 75: 1443. Available at: http://www.researchgate.net/publication/250269704_Rapid_Screening_Method_to_ Detect_Potential_Biparental_Inheritance_of_Plastid_DNA_and_Results_for_Over_ 200_Angiosperm_Species [Accessed March 17, 2015].

Csilléry, K., M.G.B. Blum, O.E. GagGiotti, and O. FrançOIS. 2010. Approximate Bayesian Computation (ABC) in practice. Trends in Ecology and Evolution 25: $410-418$. 
EDWARDS, A.W.F. 1971. Distances between Populations on the Basis of Gene Frequencies. Biometrics 27: 873-881. Available at: http://www.jstor.org/stable/2528824 [Accessed March 24, 2017].

Gardner, E.M., K.M. LARicchia, M. Murphy, D. Ragone, B.E. SCHEFfler, S. SIMPSON, E.W. WILliams, and N.J.C. ZEREGA. 2015. Chloroplast microsatellite markers for Artocarpus (Moraceae) developed from transcriptome sequences. Applications in Plant Sciences 3: apps.1500049. Available at: http://dx.doi.org/10.3732/apps.1500049 [Accessed March 28, 2017].

GARRISON, E., and G. MARTH. 2012. Haplotype-based variant detection from short-read sequencing. Available at: http://arxiv.org/abs/1207.3907 [Accessed January 25, 2017].

Gasbarra, D., S. Kulathinal, M. PIRINEN, and M.J. SillanPÄÄ. 2011. Estimating haplotype frequencies by combining data from large DNA pools with database information. IEEE/ACM Transactions on Computational Biology and Bioinformatics 8: 36-44. Available at: http://www.ncbi.nlm.nih.gov/pubmed/21071795.

GODOY, J.A., and P. JORDANO. 2001. Seed dispersal by animals: Exact identification of source trees with endocarp DNA microsatellites. Molecular Ecology 10: 2275-2283. Available at: http://doi.wiley.com/10.1046/j.0962-1083.2001.01342.x [Accessed March 22, 2017].

GorChOv, D.L., F. CORneJo, C. AsCorRA, and M. JARAMiLlo. 1993. The role of seed dispersal in the natural regeneration of rain forest after strip-cutting in the peruvian 
amazon. Vegetatio 107: 339-349. Available at:

http://www.jstor.org/stable/20046318 [Accessed March 22, 2017].

Hartl, D.L., A.G. Clark, and A.G. Clark. 1997. Principles of population genetics. Sinauer associates Sunderland.

Howe, H., and J. SMALLWOOD. 1982. Ecology of seed dispersal.

JOMBART, T. 2008. adegenet: a R package for the multivariate analysis of genetic markers. Bioinformatics 24: 1403-1405. Available at: https://academic.oup.com/bioinformatics/articlelookup/doi/10.1093/bioinformatics/btn129 [Accessed March 16, 2017].

JOSHI, N., and J. FASS. 2011. Sickle: A sliding-window, adaptive, quality-based trimming tool for FastQ files (Version 1.33) [Software]. Available at https://github.com/najoshi/sickle.2011.

KAYS, R., P.A. JANSEN, E.M.H. KNECHT, R. VOHWINKEL, and M. WiKELSKI. 2011. The effect of feeding time on dispersal of Virola seeds by toucans determined from GPS tracking and accelerometers. Acta Oecologica 37: 625-631. Available at: http://ac.els-cdn.com/S1146609X1100107X/1-s2.0-S1146609X1100107Xmain.pdf?_tid=46e2ca44-0f43-11e7-86fa00000aacb362\&acdnat=1490216908_1d364bc5aadf4cdf2c6b851d2924832f [Accessed March 22, 2017].

KiRKPATRICK, B., C.S. ARMENDARIZ, R.M. KARP, and E. HALPERIN. 2007. HaploPool: Improving haplotype frequency estimation through DNA pools and phylogenetic modeling. Bioinformatics 23: 3048-3055. 
Kofler, R., A.J. Betancourt, and C. Schlötterer. 2012. Sequencing of Pooled DNA Samples (Pool-Seq) Uncovers Complex Dynamics of Transposable Element Insertions in Drosophila melanogaster. PLoS Genetics 8: e1002487. Available at: http://dx.plos.org/10.1371/journal.pgen.1002487.

KofLER, R., R.V. PANDEY, and C. SCHLÖTtERER. 2011. PoPoolation2: Identifying differentiation between populations using sequencing of pooled DNA samples (Pool-Seq). Bioinformatics 27: 3435-3436.

Kollmann, J., and D. GoETZE. 1998. Notes on seed traps in terrestrial plant communities. Flora 193: 31-40. Available at: https://www.researchgate.net/profile/Johannes_Kollmann/publication/277709183_N otes_on_seed_traps_in_terrestrial_communities/links/559ab47608ae5d8f3937eaf3.p df [Accessed March 22, 2017].

LI, B.B., J. MorRIS, and E.B. MARTIN. 2002. Model selection for partial least squares regression. Chemometrics Intell. Lab. Syst. 64: 79-89. Available at: http://ac.elscdn.com/S0169743902000515/1-s2.0-S0169743902000515main.pdf?_tid=cee90502-102e-11e7-b17f00000aacb361\&acdnat=1490318068_848534ed2af579297aa9d6b100601ef8 [Accessed March 23, 2017].

LI, H., and R. DURBIN. 2009. Fast and accurate short read alignment with BurrowsWheeler transform. Bioinformatics.

MarTIN, M. 2011. Cutadapt removes adapter sequences from high-throughput sequencing reads. EMBnet.journal 17: 10. Available at: 
http://journal.embnet.org/index.php/embnetjournal/article/view/200 [Accessed November 14, 2016].

Martins, N.E., V.G. FAria, V. Nolte, C. Schlötterer, L. TeiXeira, É. SuCEnA, and S. MAGALHÃES. 2014. Host adaptation to viruses relies on few genes with different cross-resistance properties. Proceedings of the National Academy of Sciences of the United States of America 111: 5938-43. Available at:

http://www.ncbi.nlm.nih.gov/pubmed/24711428 [Accessed March 23, 2017].

McKenna, A., M. Hanna, E. Banks, A. Sivachenko, K. Cibulskis, A. Kernytsky, K. GARIMELLA, ET AL. 2010. The genome analysis toolkit: A MapReduce framework for analyzing next-generation DNA sequencing data. Genome Research.

MEIRMANS, P.G. 2006. Using the AMOVA framework to estimate a standardized genetic differentiation measure. Evolution 60: 2399-2402. Available at: http://dx.doi.org/10.1554/05-631.1 [Accessed March 27, 2017].

Mouissie, A.M., C.E.J. Van Der Veen, G.F. (Ciska) Veen, and R. VAn Diggelen. 2005. Ecological correlates of seed survival after ingestion by Fallow Deer. Functional Ecology 19: 284-290. Available at: http://doi.wiley.com/10.1111/j.02698463.2005.00955.x [Accessed May 4, 2015].

NAthan, R., and H.C. MulleR-LANDAU. 2000. Spatial patterns of seed dispersal, their determinants and consequences for recruitment. Trends in Ecology and Evolution 15: 278-285. Available at: http://linkinghub.elsevier.com/retrieve/pii/S0169534700018747 [Accessed March 22, 2017]. 
NEI, M. 1973. Analysis of gene diversity in subdivided populations. Proceedings of the National Academy of Sciences of the United States of America 70: 3321-3323.

PALMER, J.D. 1987. Chloroplast DNA evolution and biosystematic uses of chloroplast DNA variation. American Naturalist 130: S6-S29. Available at: http://www.jstor.org/stable/2461917 [Accessed March 22, 2017].

PE'ER, I., and J.S. BECKMANN. 2003. Resolution of haplotypes and haplotype frequencies from SNP genotypes of pooled samples. Proceedings of the seventh annual international conference on Computational molecular biology - RECOMB '03237246. Available at: http://dl.acm.org/citation.cfm?id=640075.640107.

Pritchard, J.K., M. StePhens, and P. DONNELly. 2000. Inference of population structure using multilocus genotype data. Genetics 155: 945-959. Available at: http:/ [Accessed March 16, 2017].

RAJ, A., M. StePhens, and J.K. PRITCHARD. 2014. FastSTRUCTURE: Variational inference of population structure in large SNP data sets. Genetics 197: 573-589. Available at: http://web.stanford.edu/group/pritchardlab/publications/pdfs/Raj14 [Accessed March 16, 2017].

Sboner, A., X. Mu, D. Greenbaum, R.K. Auerbach, M.B. Gerstein, M. MetzKer, E. MARDIS, ET AL. 2011. The real cost of sequencing: higher than you think! Genome Biology 12: 125. Available at: http://genomebiology.com/2011/12/8/125 [Accessed March 22, 2017].

SChlÖtterer, C., R. Tobler, R. Kofler, and V. Nolte. 2014. Sequencing pools of individuals — mining genome-wide polymorphism data without big funding. Nature 
Reviews Genetics 15: 749-763. Available at:

http://www.nature.com/doifinder/10.1038/nrg3803 [Accessed March 23, 2017].

Sham, P., J.S. BADER, I. Craig, M. O’Donovan, and M. Owen. 2002. DNA Pooling: a tool for large-scale association studies. Nat Rev Genet 3: 862-871. Available at: http://www.nature.com/nrg/journal/v3/n11/pdf/nrg930.pdf [Accessed March 22, 2017].

Sims, D., I. Sudbery, N.E. IlotT, A. Heger, and C.P. PonTing. 2014. Sequencing depth and coverage: key considerations in genomic analyses. Nature reviews. Genetics 15: 121-32. Available at: http://dx.doi.org/10.1038/nrg3642 [Accessed July 11, 2014].

SlatKIN, M. 1987. Gene Flow and the Geographic Structure of Natural Populations. Science 236: 787-792. Available at: http://science.sciencemag.org/content/236/4803/787 [Accessed March 22, 2017].

Stull, G.W., M.J. Moore, V.S. Mandala, N. A Douglas, H.-R. Kates, X. QI, S.F. BROCKINGTON, ET AL. 2013. A targeted enrichment strategy for massively parallel sequencing of Angiosperm plastid genomes. Applications in Plant Sciences 1: 1-7. Available at: http://www.bioone.org/doi/abs/10.3732/apps.1200497.

Templeton, A.R. 1998. Nested clade analyses of phylogeographic data: Testing hypotheses about gene flow and population history. Molecular Ecology 7: 381-397. Available at: http://doi.wiley.com/10.1046/j.1365-294x.1998.00308.x [Accessed February 7, 2017].

Templeton, A.R. 2009. Statistical hypothesis testing in intraspecific phylogeography: Nested clade phylogeographical analysis vs. approximate Bayesian computation. 
Molecular Ecology 18: 319-331.

Templeton, A.R., K.A. Crandall, and C.F. Sing. 1992. A cladistic analysis of phenotypic associations with haplotypes inferred from restriction endonuclease mapping and DNA sequence data. III. Cladogram estimation. Genetics 132: 619633. Available at: https://www.ncbi.nlm.nih.gov/pmc/articles/PMC1205162/pdf/ge1322619.pdf [Accessed March 28, 2017].

ThORVAldsdótTIR, H., J.T. Robinson, and J.P. MeSIROV. 2013. Integrative Genomics Viewer (IGV): High-performance genomics data visualization and exploration. Briefings in Bioinformatics.

Trakhtenbrot, A., R. NAthan, G. Perry, and D.M. Richardson. 2005. The importance of long-distance dispersal in biodiversity conservation. Diversity and Distributions 11: 173-181. Available at: http://doi.wiley.com/10.1111/j.13669516.2005.00156.x [Accessed February 7, 2017].

WALKER, J.F., M.J. ZANIS, and N.C. EMERY. 2014. Comparative analysis of complete chloroplast genome sequence and inversion variation in Lasthenia burkei (Madieae, Asteraceae). American journal of botany 101: 722-9. Available at: http://www.amjbot.org/content/101/4/722.long [Accessed April 20, 2015].

Whitlock, M.C. 2011. G'ST and D do not replace FST. Molecular Ecology 20: 10831091. Available at: http://doi.wiley.com/10.1111/j.1365-294X.2010.04996.x. WILLSON, M.F. 1993. Dispersal mode, seed shadows, and colonization patterns. Vegetatio 107-108: 261-280. 
WRIGHT, S. 1949. The genetical structure of populations. Annals of Eugenics 15: 323354. Available at: http://doi.wiley.com/10.1111/j.1469-1809.1949.tb02451.x [Accessed December 1, 2014]. 


\section{Appendix A: CallHap Bioinformatics Pipeline Overview}

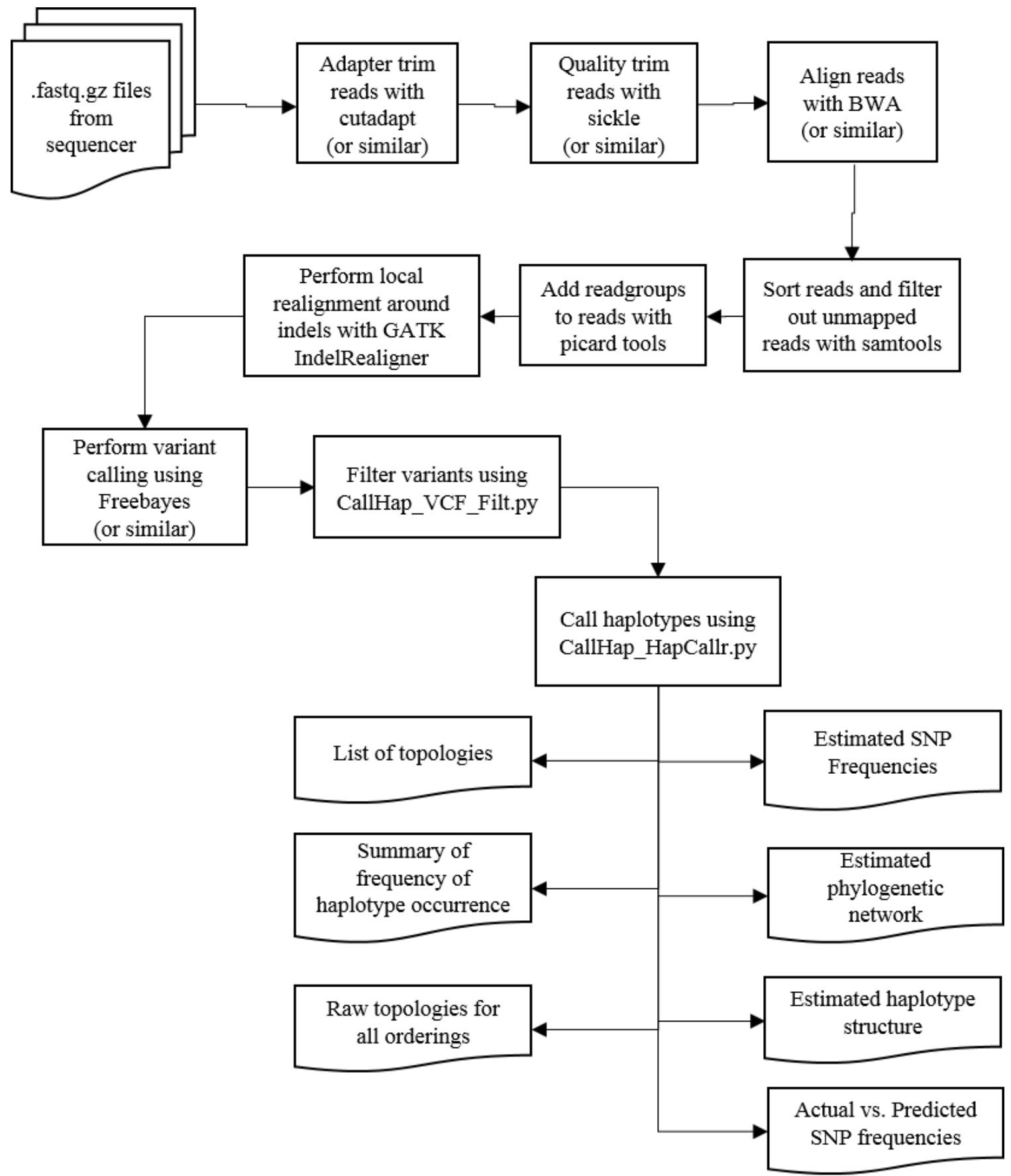


A MYbaits-3 custom cpDNA capture array from MYcroarray (MYcroarray, Ann Arbor, MI, USA) was created to help isolate cpDNA. During capture array creation, 120mer baits were constructed with a $\sim 2 x$ flexible tiling density. Any baits with 10 or less mismatches between them were collapsed into a single bait. In total, the capture array contained 55,409 baits.

Species

Source

Achillea millefolium

Achyrachaena mollis

Partial De Novo

Arabis alpa

Partial De Novo

Brachypodium distachyon

NCBI: NC_023367.1

Camassia quamash

NCBI: NC_011032.1

Chrysanthemum indicum

Partial De Novo

Cryptantha torreyana

NCBI: NC_020320.1

Danthonia californica

NCBI: KP096524.1

Danthonia californica

NCBI: NC_025232.1

Eriophyllum lanatum

Partial De Novo

Eustrephus latifolius

Partial De Novo

Festuca arundinacea

NCBI: NC_025305.1

Festuca roemeri

NCBI: NC_011713_2

Fragaria vesca

Partial De Novo

Lactusa sativa

NCBI: NC_015206.1

Lasthenia burkei

NCBI: NC_007578.1

Lomatium utriculatum

NCBI: KM360047.1

Lupinus albus

Lupinus bicolor

Nama carnosum

Partial De Novo

NCBI: NC_026681.1

Nicotina undulata

Partial De Novo

Private communication with Gregory Stull

Petroselinium crispum

NCBI: NC_016068.1

Quercus aliena

NCBI: HM596073.1

Ranunculus austro-oreganus

NCBI: KP301144.1

Ranunculus macranthus

Partial De Novo

Ranunculus occidentalis

NCBI: NC_008796.1

Salvia miltiorrhiza

Partial De Novo

Hibiscus syriacus

NCBI: NC_020431.1

NCBI: NC_026909.1

Oenothera biennis

NCBI: NC_010361.1

Lonicera japonica

NCBI: NC_026839.1

Lilium superbum

NCBI: NC_026787.1

Primula poissonii

NCBI: NC_024543.1

Liquidambar formosana

NCBI: NC_023092.1 


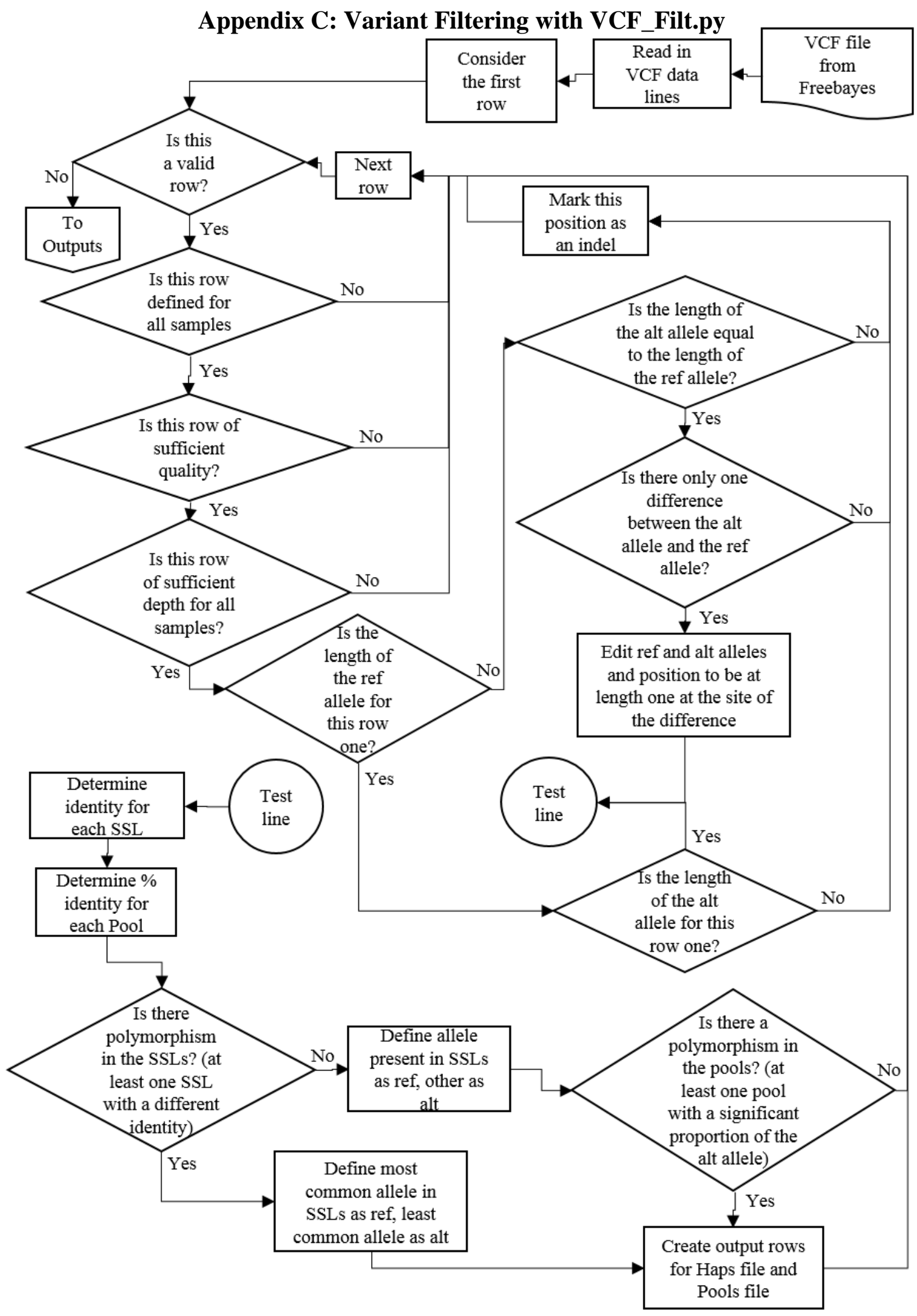




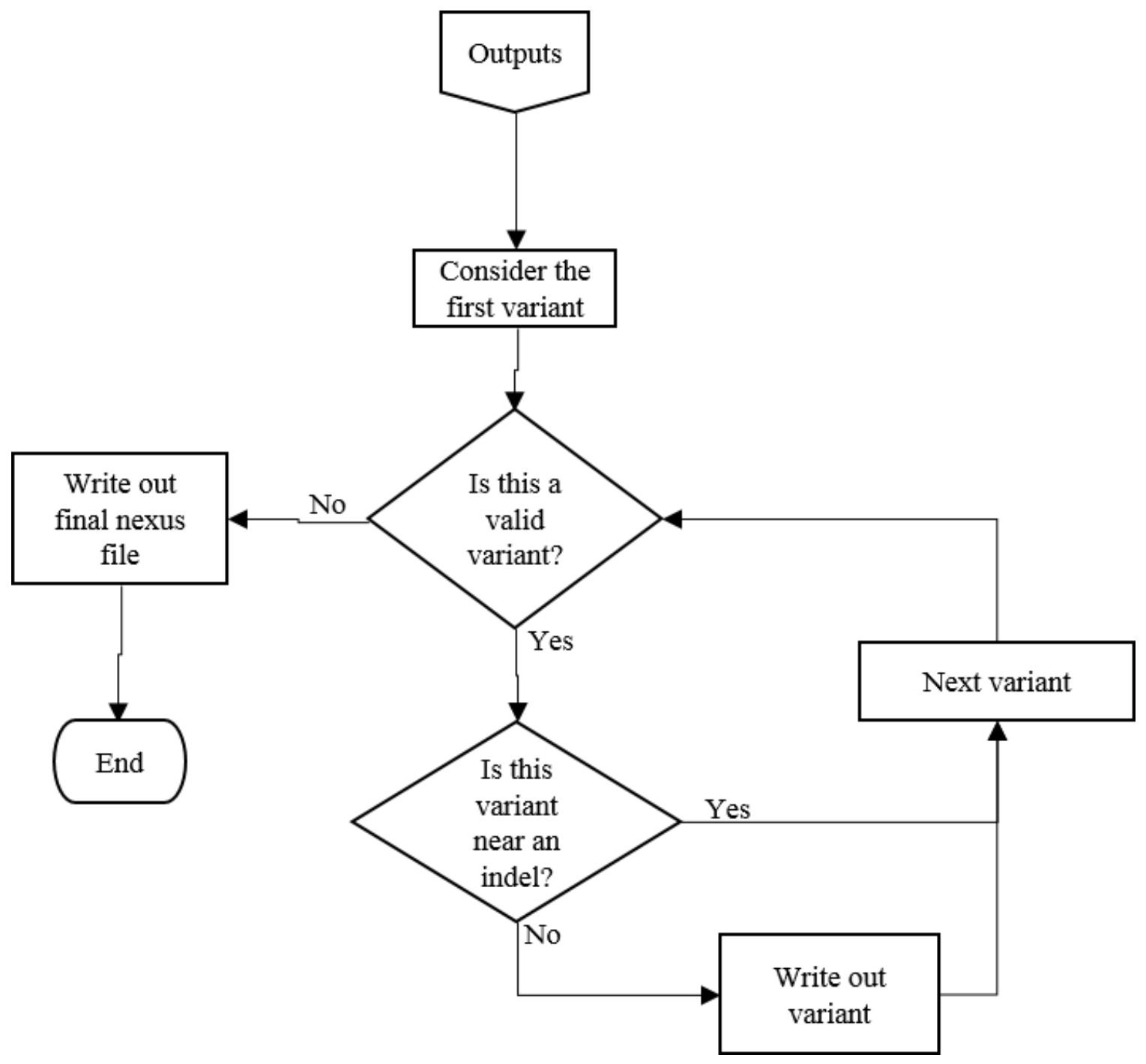




\section{Appendix D: Overall haplotype and frequency estimation program (HapCallr.py)}

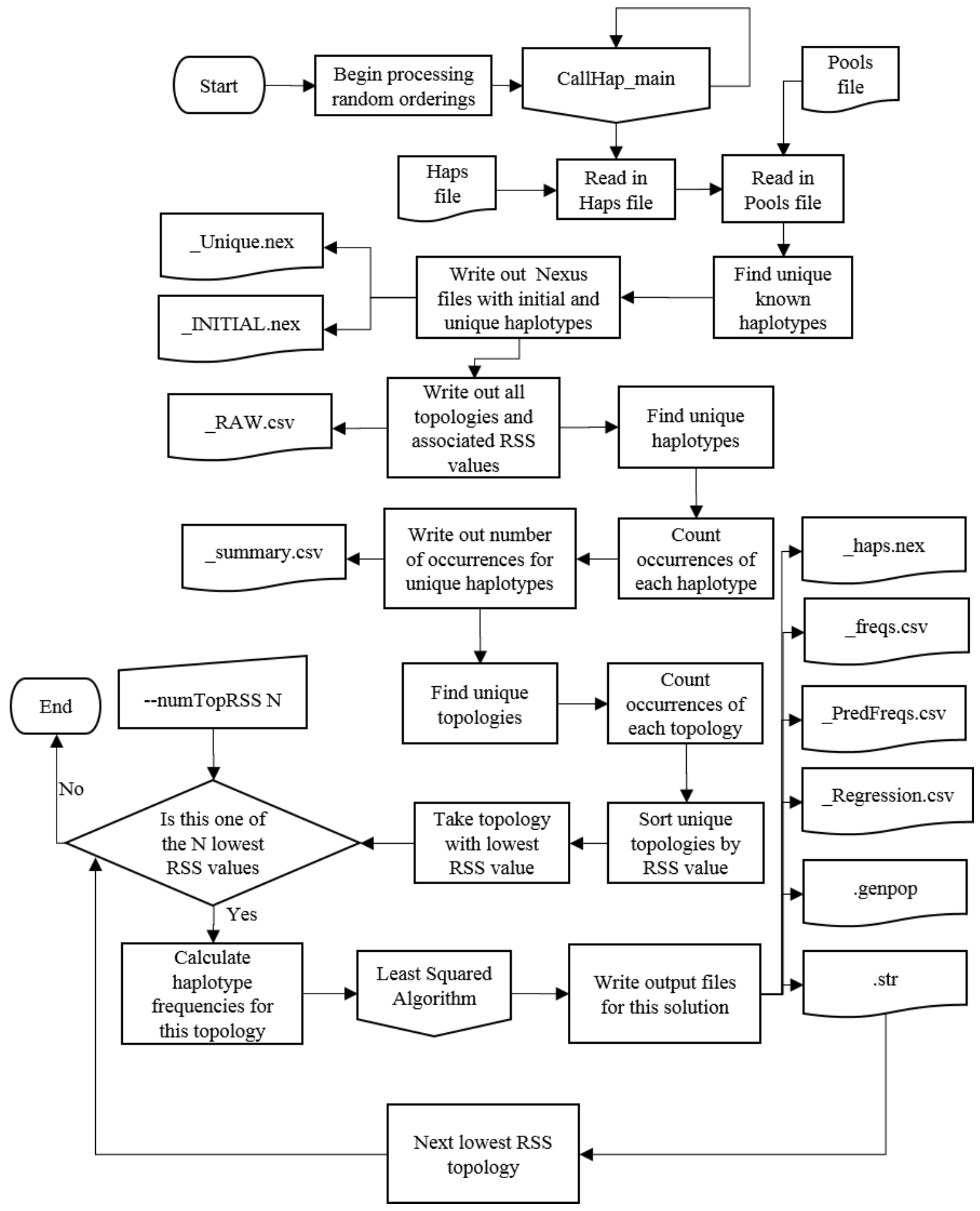




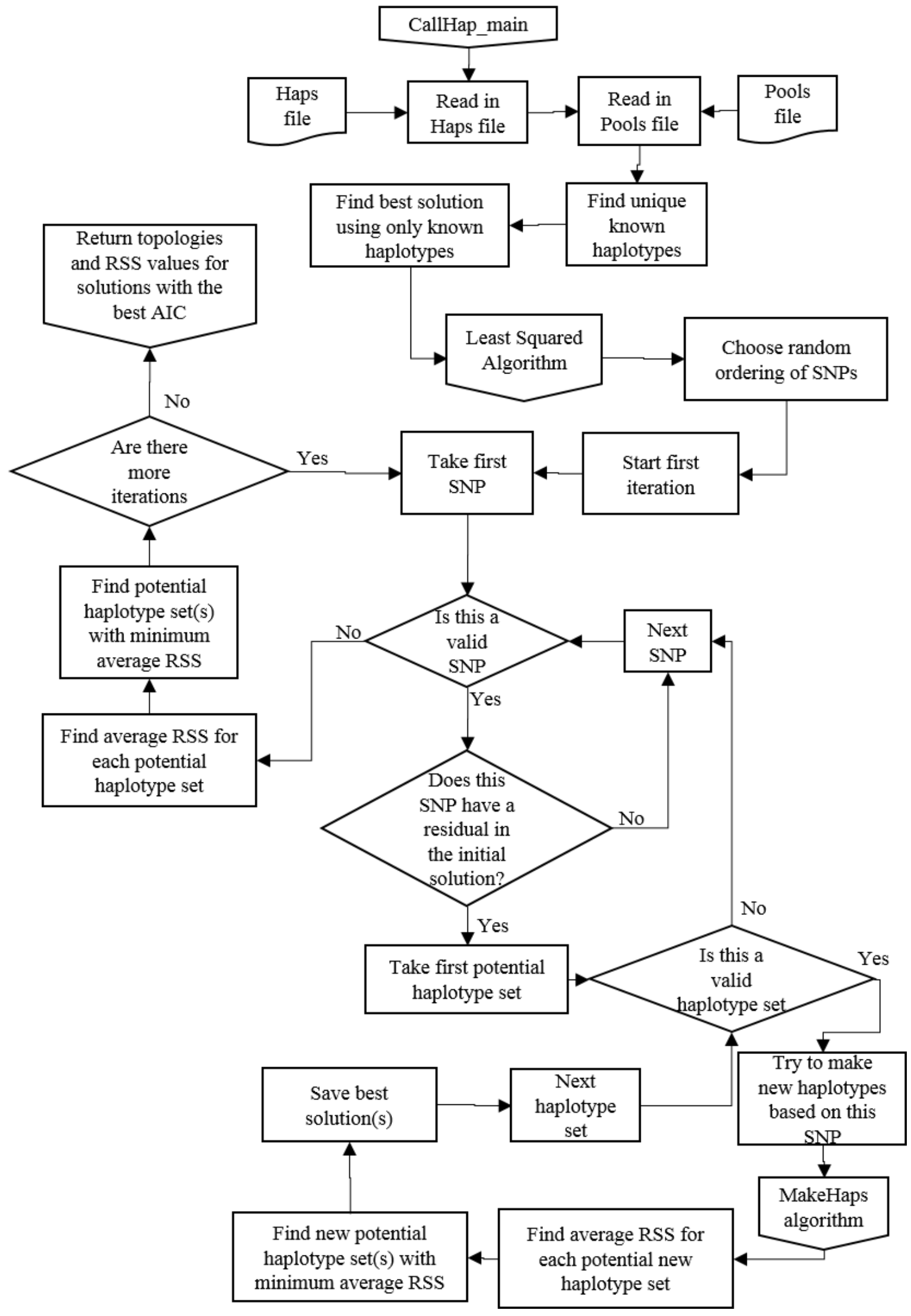




\section{Appendix E: CallHap Least Squares Algorithm}

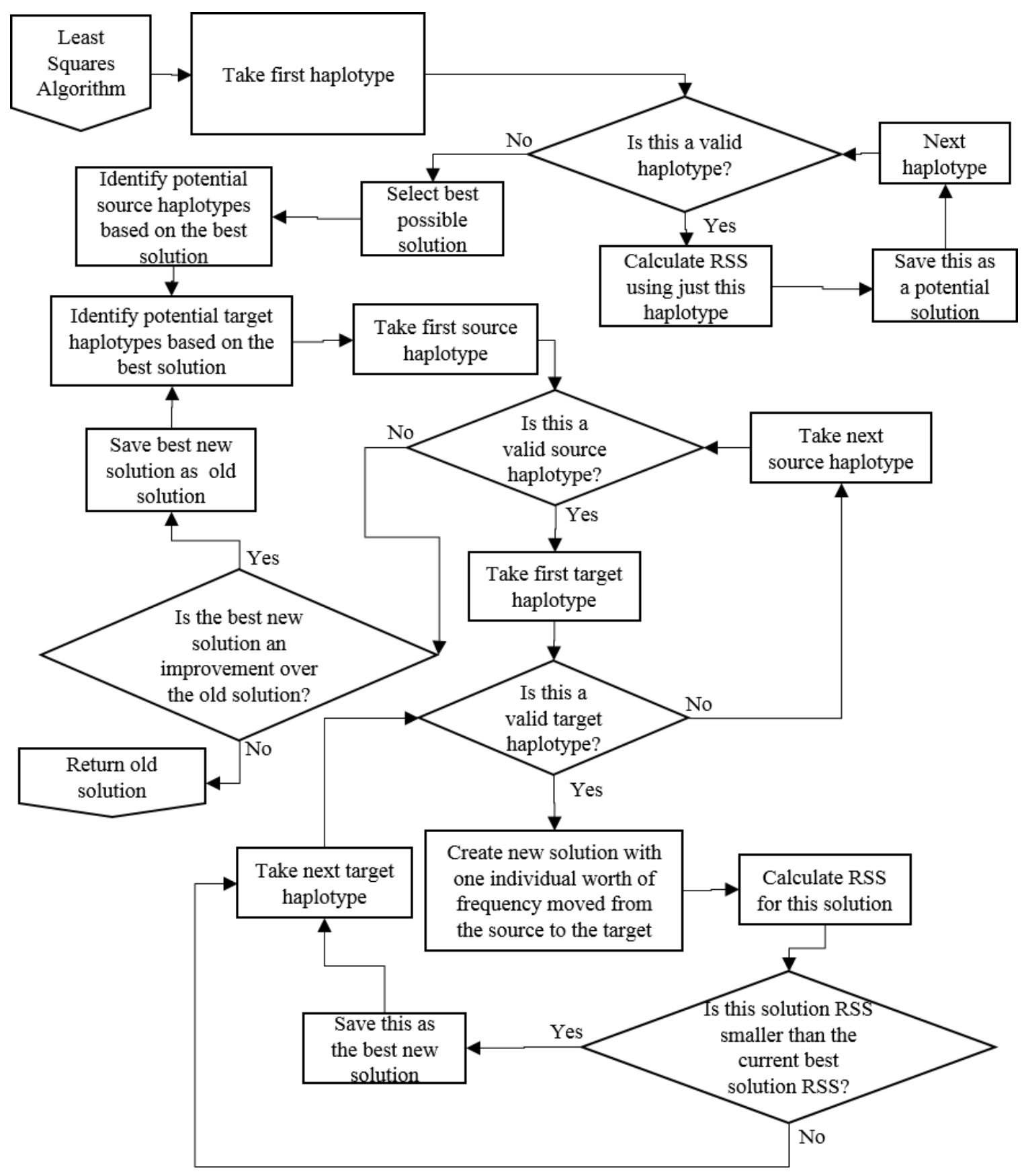




\section{Appendix F: CallHap Haplotype Creation Algorithm}

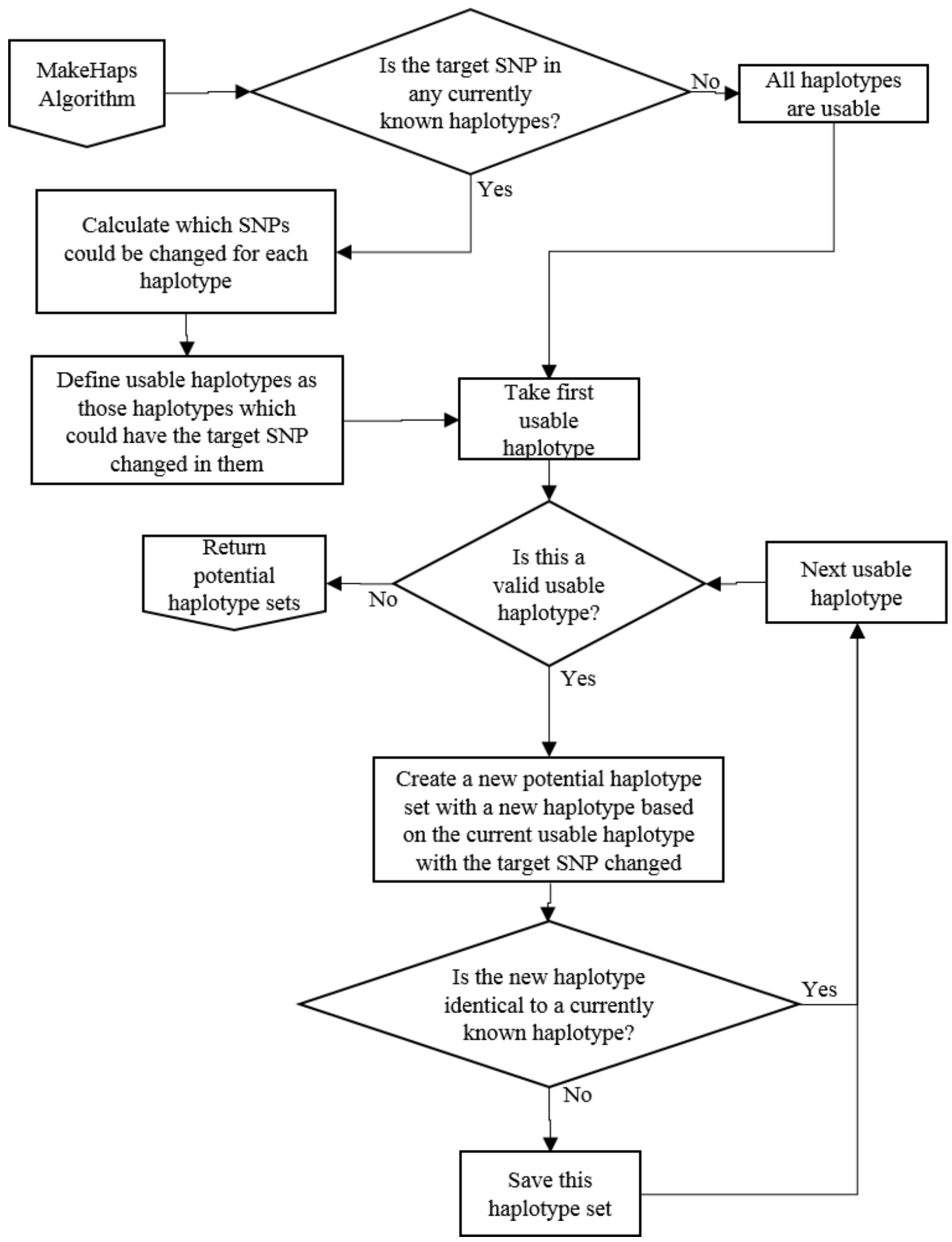




\section{Appendix G: CallHap Manual}

\section{CallHap: A Pipeline for Analysis of Pooled Whole-genome Haplotypes}

Last edited: 04/28/2017 By: Jessica Persinger

\section{Licensing information}

With the exception of the Genome Analysis Toolkit, all programs are freely available under either the Gnu Public License or the MIT License. The Genome Analysis Toolkit is free for non-commercial use; other use should contact the Broad Institute at softwarelicensing@broadinstitute.org. Python and bash scripts for the CallHap pipeline are available at https://github.com/cruzan-lab/CallHap.

\section{Introduction}

CallHap is a pipeline designed for the analysis of pooled haplotype data. It depends on the presence of two types of sequencing libraries; either single sample libraries (SSLs) or pooled libraries (Pool). Ideally, a Pool should contain equimolar genetic material from 20 individuals, and one of those individuals should be prepared separately as a SSL. This pipeline picks up following sequencing on an Illumina HiSeq or similar high-throughput sequencer.

\section{Requirements}

- A LINUX/UNIX/MacOS system with the following programs installed:

- Cutadapt (http://cutadapt.readthedocs.io/en/stable/index.html)

- Sickle (http://bioinformatics.ucdavis.edu/research-computing/software/)

- BWA (http://bio-bwa.sourceforge.net/)

- Samtools (http://samtools.sourceforge.net/)

- PicardTools (https://broadinstitute.github.io/picard/)

- GATK (https://software.broadinstitute.org/gatk/)

- Freebayes (https://github.com/ekg/freebayes)

- Python 2.7x (https://www.python.org/) with NumPy (http://www.numpy.org/)

- Java Development Kit 


\title{
Contents
}

\author{
Quick start \\ Setup \\ Preprocessing \\ SNP Calling \\ SNP Filtering \\ SNP Calling \\ Haplotype Calling \\ Detailed Instructions \\ Adapter/Quality Trimming \\ Read Alignment \\ Readgroup Creation \\ Local Realignment \\ SNP Calling \\ SNP Filtering \\ Haplotype Calling
}




\section{Quick Start}

\section{Setup:}

program-config.sh:

Edit program-config.sh so that each of the variables is set to the absolute path of the program in question.

Reference Preparation:

Obtain a reference genome (in FASTA format) for your species of interest (or closely related other species), and prepare it for use by using the following commands:

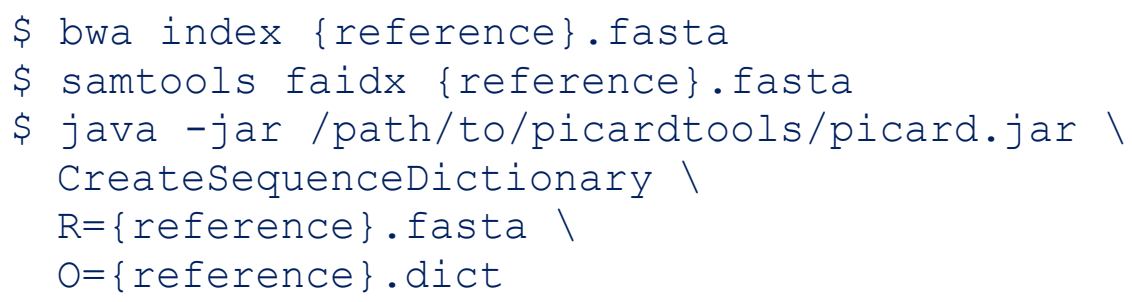

\section{Preprocessing:}

Note that there are two basic processing pipelines provided; one with automated trimming (CallHap_Preproc_0.01.23.sh) and one without automated trimming (CallHap_Preproc_NoTrimming_0.01.23.sh). It is strongly suggested that at least a few (2-5) samples per flow cell be run manually (one step at a time), at least through trimming for quality control and to see if those samples need any additional trimming beyond the basic trimming steps (adapter and quality trimming). If you are doing trimming separately, be sure to use the locations of the trimmed files in the preconfig instead of the locations of the raw files.

Create a preconfig file in Excel with the following columns:

- Read1File

- Read2File

- RGLB

- RGSM

- RGPU

- Mode

- Reference

Each row should represent one sequencing library (SSL or Pooled).

- Read1File and Read2File should give the absolute path to the locations of the raw data for the Read 1 and Read 2 files (in the case of single end data, give the file location under Read1File, and put a period (.) for Read2File).

- RGLB should be some identifier for the library (e.g. library number).

- RGSM should be a sample name, preferably indicating the species of the library, the location the sample came from, and whether the sample is a SSL or Pool (Example: SpenamLocS\#SSL, SpenamLocS\#Pool). 
- RGPU should indicate the barcoding used for this library during library prep (Example: ATTACTCG-TATAGCCT).

- Mode should be one of se (single-end) or pe (paired-end).

- Reference should indicate the reference genome you would like this library aligned to.

If all samples are of the same species, the reference genomes for all libraries should be the same.

Save the preconfig file as a .csv. Convert it to a config file using:

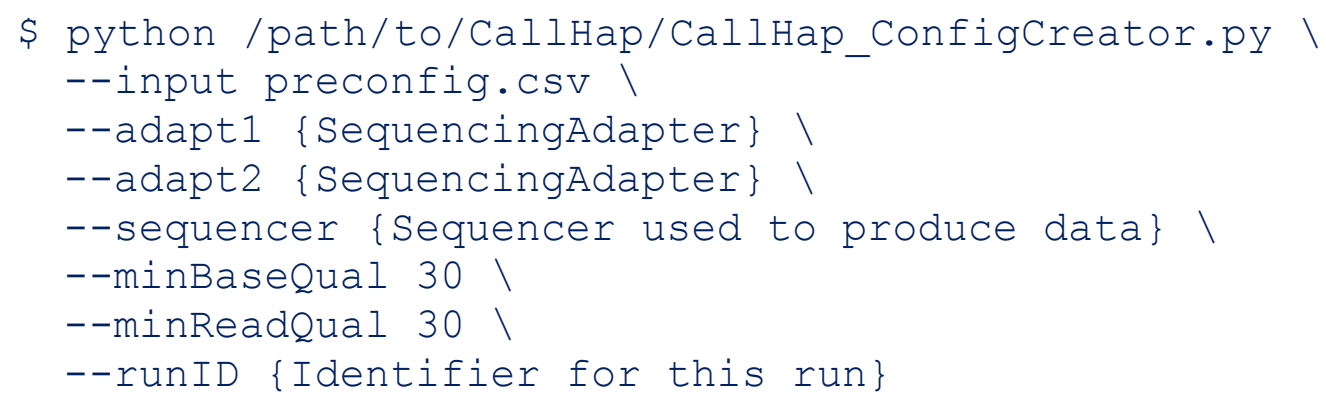

This will output a .sh file with the run ID as the name (for example, of you put --runID $\{$ runID\}, the file would be called runID.sh)

Then use the following command to run the rest of the pre-processing (replacing the script name if you did trimming separately):

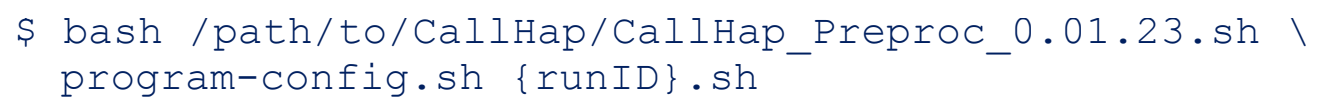

\section{SNP Calling:}

Set up an input list of files using:

$$
\begin{aligned}
& \text { \$Is -1/path/to/files/*SSL*rg.ra.bam > \{RunID\}.txt } \\
& \text { \$ Is -1/path/to/files/*Pool*.rg.ra.bam >> \{RunID\}.txt }
\end{aligned}
$$

Call FreeBayes using:

$$
\begin{aligned}
& \text { \$ / path/to/freebayes/freebayes -L \{RunID\}.txt । } \\
& \text {-p } 1 \text {-f /path/to/reference/\{reference\}.fasta I } \\
& -\mathrm{V}\{\text { RunID\}_SNPs.vcf --use-best-n-alleles } 2 \backslash \\
& \text {--min-repeat-entropy } 1 \text {--no-partial-observations } \mid \\
& \text {--min-alternate-fraction }\{0.05\}
\end{aligned}
$$

$$
\begin{aligned}
& \text {--min-alternate- } \\
& \text { fraction }
\end{aligned}
$$

Should be set to 1/poolsize or lower. 
This step may take a while, and while running, may look like it isn’t doing anything

\section{SNP Filtering:}

SNP filtering is accomplished by use of a custom python script, which can be run with the command:

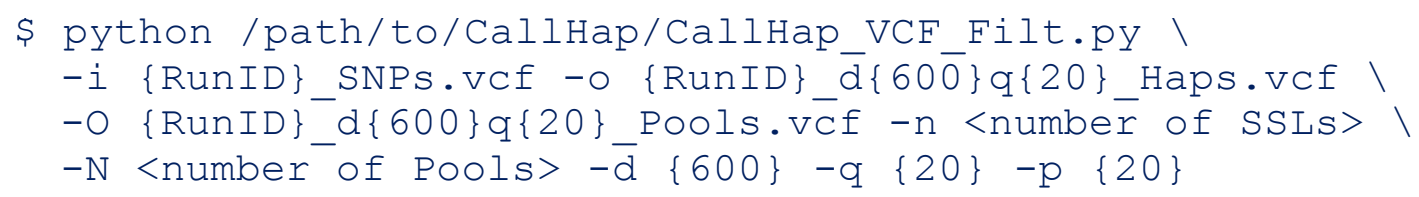

You may need to trim off one or more columns from the VCF file if one sample was not called at a majority of positions; if a single sample is not called at a particular position, the variant at that position will be discarded. To determine if a column needs to be removed, look at your VCF file in Excel, and see if there are any columns that are periods (“.") for the majority of rows. Removing the column can also be done in Excel, but you need to be careful because Excel likes to add quotes when it saves files with commas in the cells, as do most spreadsheet editors I've found.

\section{Haplotype Calling:}

Before running this step, check how many cores are available on the system you're using with htop. Make sure you don't overload the system you're working on; don't set -t to higher than the number of available cores, and don't take up all the cores on the machine.

Haplotype calling can be run using:

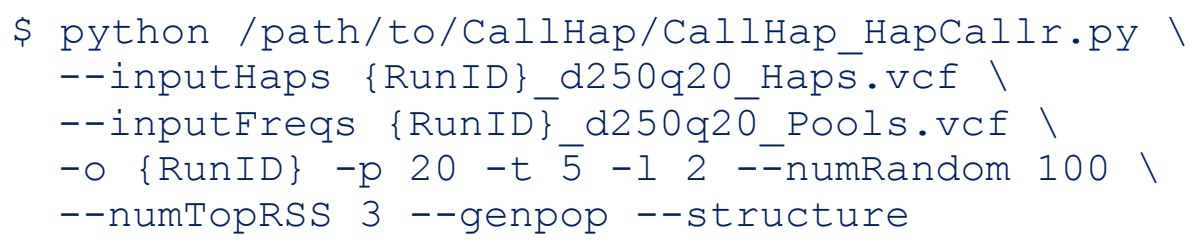

This program generates four to six output file per solution output (within the minimum number of RSS values):

- A NEXUS file (RunID_solNum_haps.nex) for network phylogeny creation; PopART (http://popart.otago.ac.nz/index.shtml) works fairly well. I've been using the TCS algorithm.

- A VCF file (RunID_solNum_PredFreqs.vcf) containing the estimated SNP frequencies based on the estimated haplotype frequencies, and the per-SNP average residuals in the INFO field

- A CSV file (RunID_solNum_freqs.csv) containing the per-pool haplotype frequencies and RSS values for each pool.

- A CSV file (RunID_solNum_Regression.csv) containing paired observed and predicted SNP frequencies from the Least Squared algorithm. 
- (Optional): A Structure formatted file (RunID_solNum_iterNum.str) containing the expanded haplotype frequencies

- (Optional): A Genpop file containing the haplotype frequencies for use in Adigenet.

In addition, outputs are generated describing the original haplotypes network (RunID_Initial.nex), the unique haplotypes network (RunID_Unique.nex), raw topologies observed from each random order (RunID_RAW.csv), the frequency of each unique topology generated (RunID_topologies.csv), the frequency of occurrence for each haplotype found in any random order (RunID_summary.csv).

In terms of population-genetics analysis, haplotypes should be treated as independent alleles at a single locus. 


\section{Detailed Instructions}

\section{Adapter/Quality trimming:}

Adapter and quality trimming should be performed before any other step in the pipeline. This ensures better read alignment and higher quality of the final data. The automated pipeline uses cutadapt for adapter trimming and sickle for quality trimming; however, you can use other trimming programs if so desired.

Cutadapt is available at http://cutadapt.readthedocs.io/en/stable/index.html under the MIT License and can be run using:

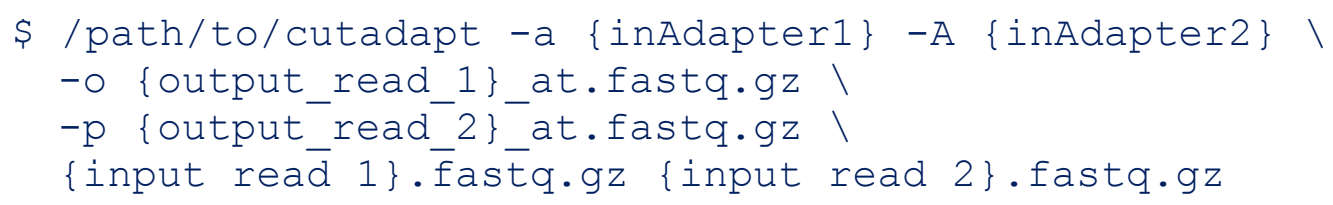

for paired-end reads or

$$
\begin{aligned}
& \text { \$ / path/to/cutadapt -a \{inAdapter } 1\} \backslash \\
& \text {-o \{output read } 1\} \text { at.fastq.gz \{input read } 1\} \text {.fastq.gz }
\end{aligned}
$$

for single-end reads.

If you aren't certain what adapter sequence you have, running FastQC (freely available at http://www.bioinformatics.babraham.ac.uk/projects/fastqc/ under GPLv3) may help determine what adapters are present. Otherwise, consult your library preparation protocol.

While cutadapt can also do quality trimming (using the -q option), or remove a fixed number of bases (using the -u option), the default pipeline uses a second program, (sickle) for quality trimming. Sickle is available at http://bioinformatics.ucdavis.edu/research-computing/software/ under the MIT License and can be run with

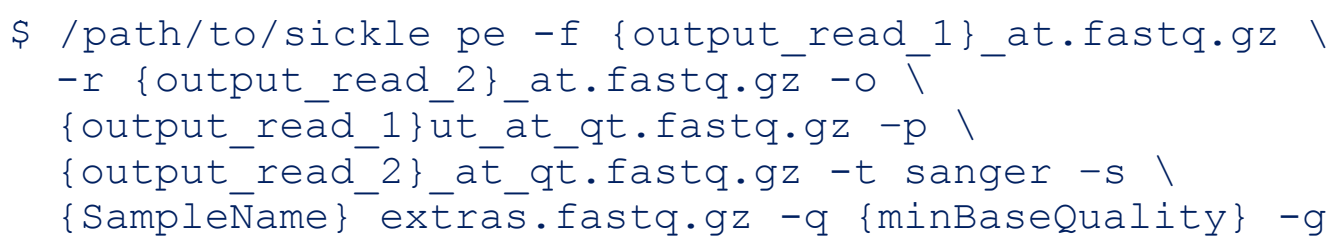

for paired-end reads or

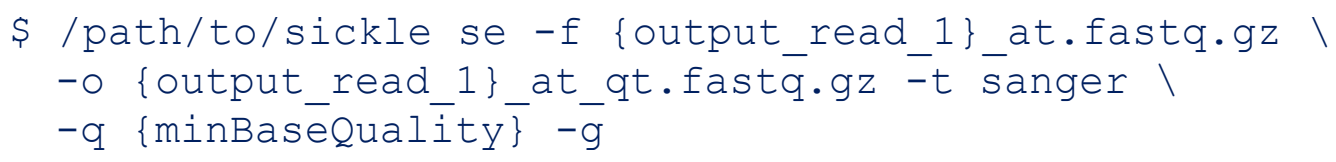


for single-end reads.

For more details on these programs, consult their respective manuals.

Following trimming, it is recommended that at least 2-5 samples per flow cell be qualitychecked using FastQC. For this pipeline, check that there are almost no remaining adapters of any type in the AdapterContent page of the report and that you are satisfied with the quality scores in the Per base sequence quality section and the base percentages in the Per base sequence content section.

Note that FastQC will generate output files in the same directory as the input files.

\section{Read alignment:}

The automated pipeline uses BWA-mem to align reads with default options. BWA can be obtained from http://bio-bwa.sourceforge.net/ under GPLv3, and can be run using:

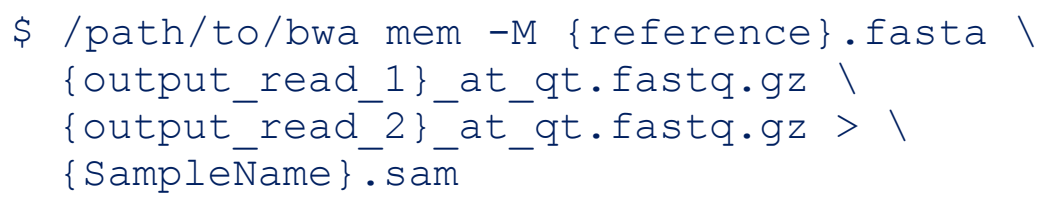

for paired-end reads or

$$
\begin{aligned}
& \text { \$/path/to/bwa mem -M \{reference }\} \text {.fasta } \backslash \\
& \{\text { output_read_1\}_at_qt.fastq.gz }>\{\text { SampleName } \text {. sam }
\end{aligned}
$$

for single-end reads.

After alignment, the file is converted to a bam file:

$$
\begin{aligned}
& \text { \$/path/to/samtools view -Sbu -F } 4 \text { \{SampleName\}.sam I I } \\
& \text { /path/to/samtools sort - } \text { SampleName\}.sort }
\end{aligned}
$$

Index the bam file:

$$
\text { \$ /path/to/samtools index \{SampleName\}.sort.bam }
$$

At this time, any unaligned reads are also removed.

Samtools can be obtained from http://www.htslib.org/.

\section{Readgroup Creation:}

PicardTools is used to add readgroups to the files. These are a requirement for local realignment with GATK, and for SNP calling with FreeBayes. For later analysis, it is useful if each sample have a different sample name (RGSM) and readgroup ID (RGID), 
since Freebayes (our SNP caller) uses the readgroup ID to differentiate samples. I used the library number as the readgroup ID.

PicardTools is available at https://broadinstitute.github.io/picard/, and can be run using

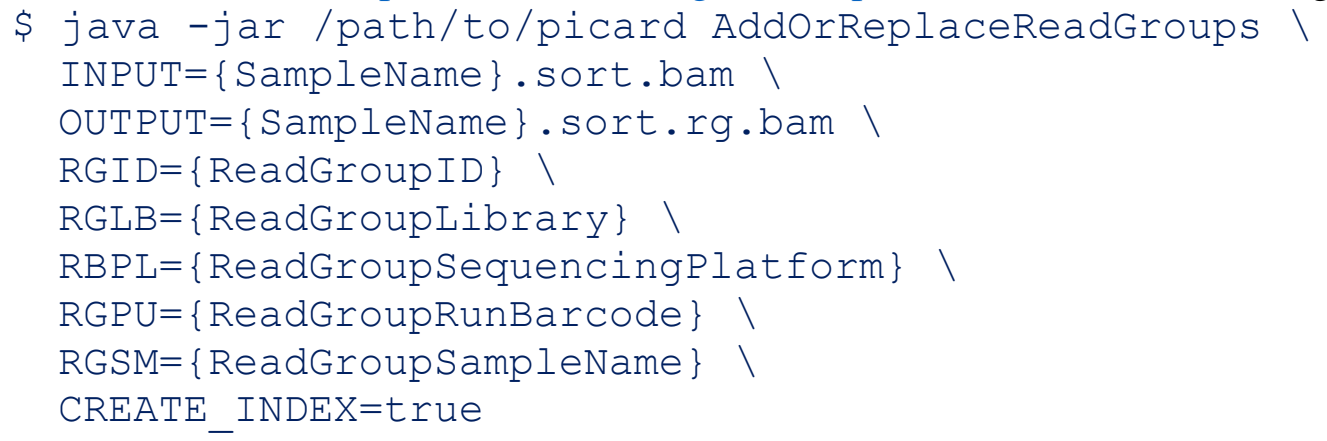

RGLB, RBPL, RGPU, and RGSM are required for this tool to run.

RGID needs to be different for each library.

\section{Local Realignment:}

Local realignment is carried out using the Genome Analysis Toolkit (GATK, available at https://software.broadinstitute.org/gatk/). The first step in this process is to locate targets for local realignment using:

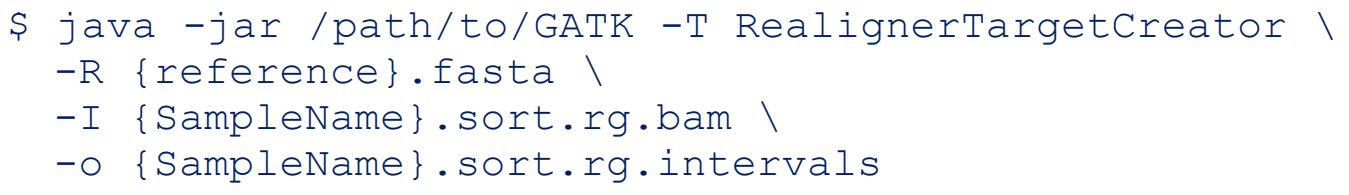

Following this, local realignment can be run using:

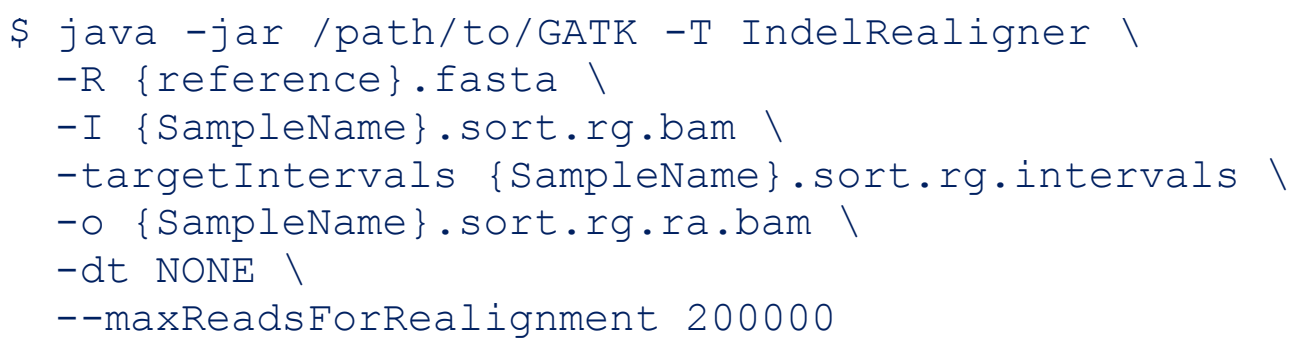

\section{SNP Calling:}

Set up an input list of files using:

$$
\begin{aligned}
& \$ \text { Is }-1 / \text { path/to/files/*SSL*.rg.ra.bam > RunID } \text {.txt } \\
& \$ \text { ls }-1 / \text { path/to/files/*Pool*.rg.ra.bam }>\text { RunID } \text {.txt }
\end{aligned}
$$


Or whatever identifier you used to differentiate PLs and SSLs. The important thing is that this file list all SSLs, followed by all PLs.

Call FreeBayes using:

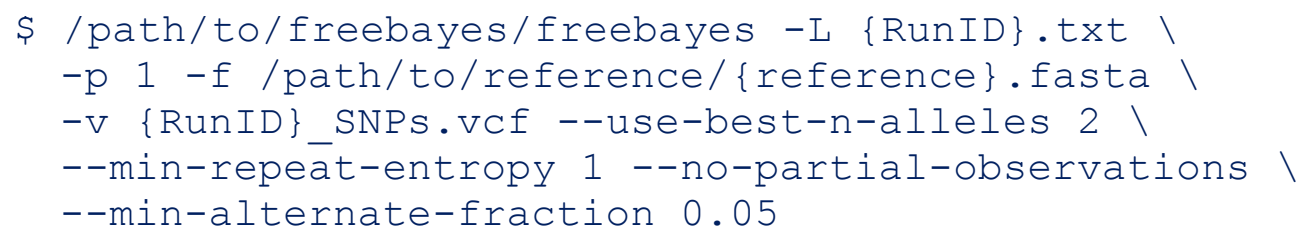

--min-alternate-

Should be set to $1 /$ poolsize or lower.

fraction

This step may take a while, and while running, may look like it isn't doing anything. FreeBayes can be found at https://github.com/ekg/freebayes.

\section{SNP Filtering:}

Before running SNP filtering, it may be necessary to trim off one or more columns from the VCF file if one sample was not called at a majority of positions; if a single sample is not called at a particular position, the variant at that position will be discarded, so a single sample uncalled (or at low depth) at a majority of positions can result in no data making it through the filtering step. To determine if a column needs to be removed, look at your VCF file in Excel, and see if there are any columns that are periods (".") for the majority of rows. Removing the column can also be done in Excel, but you need to be careful because Excel likes to add quotes when it saves files with commas in the cells, as do most spreadsheet editors I've found.

If desired, sample depth can be assessed using the GATK DepthOfCoverage tool (see https://software.broadinstitute.org/gatk/documentation/tooldocs/org broadinstitute gatk tools_walkers_coverage_DepthOfCoverage.php for instructions). This tool takes a similar amount of time to SNP calling.

SNP filtering is accomplished by use of a custom python script, which can be run with the command:

\$ python /path/to/CallHap/CallHap_VCF_Filt.py ।

$-i\{$ RunID\}_SNPs.vcf -o \{RunID\}_d600 $\bar{q} 20$ Haps.vcf ।

$-O\{$ RunID $\}$ d600q20_Pools.vcf $-\bar{n}<$ number of SSLs $>\backslash$

$-\mathrm{N}$ <number of Pools $>-\mathrm{d} 600$-q 20 -p 20

\begin{tabular}{|l|l|}
\hline$-i$ & The input VCF file from FreeBayes \\
\hline$-\circ$ & $\begin{array}{l}\text { The output haplotypes file, containing haplotypes found in the } \\
\text { SSLs }\end{array}$ \\
\hline
\end{tabular}




\begin{tabular}{|c|c|}
\hline$-\mathrm{O}$ & $\begin{array}{l}\text { The output Pool SNP frequencies file, containing frequency of } \\
\text { the more common allele in each pool }\end{array}$ \\
\hline$-\mathrm{n}$ & The number of SSLs in the input file \\
\hline$-\mathrm{N}$ & The number of Pools in the input file \\
\hline$-d, \quad--m i n D e p t h$ & $\begin{array}{l}\text { This option sets the minimum read depth that must be present } \\
\text { at a position in ALL libraries in order for that position to be } \\
\text { considered as a variant. It should be set based on the number } \\
\text { of individuals in a PL. For haploid sequence, a depth of } 15 \text { per } \\
\text { individual in the pool is recommended (Sims et al., 2014), so } \\
\text { that for a pool of } 20 \text { individuals, a depth of } 300 \text { is required at a } \\
\text { site to be able to call variants. }\end{array}$ \\
\hline$-q, \quad--m i n Q u a l$ & $\begin{array}{l}\text { Controls the minimum PHRED-scaled variant quality needed } \\
\text { to use a variant. Mostly useful for filtering out super-low } \\
\text { quality variants, but can be set higher as necessary. -p is the } \\
\text { number of individuals in each pool. }\end{array}$ \\
\hline --minCallPrev & $\begin{array}{l}\text { Controls the maximum allowable error in SSLs for a variant to } \\
\text { be called. It can range from } 1 \text { (all reads in each SSL need to } \\
\text { have the same identity) to } 0.5 \text { (Up to half the reads in a SSL } \\
\text { can have a different identity). At a setting of } 1 \text {, some real } \\
\text { SNPs could be removed based on unavoidable errors in the } \\
\text { SSLs, while at a setting of } 0.5 \text {, confidence in the identity call } \\
\text { for SSLs, and thus in the identity of haplotypes, will be } \\
\text { significantly decreased. I set this parameter at a default of } 0.9 \text {, } \\
\text { to allow for some sequencing error in the SSLs while still } \\
\text { maintaining a high accuracy of SSL identity calls. }\end{array}$ \\
\hline --minSnpPrev & $\begin{array}{l}\text { Coupled with poolSize, this option controls how much of a PL } \\
\text { must be the alternate identity for a SNP to be at that position } \\
\text { when there is no variation in the SSLs. The value is a positive } \\
\text { floating-point decimal, which gets multiplied by } 1 / \text { poolSize to } \\
\text { yield the proportion of reads that must be of a different identity } \\
\text { in a PL to yield a variant. At a value of zero, all positions } \\
\text { would be called as variants if there was any variation in a PL. } \\
\text { I set this at a default value of } 0.75 \text { in order to allow for some } \\
\text { error in low-frequency haplotypes, while removing the } \\
\text { majority of low-frequency sequencing errors from } \\
\text { consideration. }\end{array}$ \\
\hline --indelDist & $\begin{array}{l}\text { How far away from indels a variant should be for use. } \\
\text { IndelDist takes an integer value greater than } 0 \text {; at a value of } 0 \text {, } \\
\text { distance from an indel will not be considered as a filter. I set } \\
\text { this at a relatively conservative value of } 100 \text { (the length of my } \\
\text { raw sequencing reads) as being the maximum distance at } \\
\text { which the presence of an indel could have any effect on variant } \\
\text { discovery. }\end{array}$ \\
\hline
\end{tabular}


It is recommended to run this program with different sets of parameters to determine what the optimum parameters will be for a particular run.

\section{Haplotype Calling:}

Before running this step, check how many cores are available on the system you're using with htop. Make sure you don't overload the system you're working on; don't set -t to higher than the number of available cores, and don't take up all the cores on the machine.

Haplotype calling can be run using:

\$ python /path/to/CallHap/CallHap_HapCallr.py I --inputHaps \{RunID\}_d250q20_Haps.vef ।

--inputFreqs $\left\{\right.$ RunID ${ }_{-}$d250q20_Pools.vCf ।

- 0 \{RunID $\}$-p 20 -t $\overline{5}-12$--numRandom 100 -numTopRSS 3

\begin{tabular}{|c|c|}
\hline --inputHaps & The haplotypes file from SNP filtering \\
\hline --inputFreqs & The Pools file from SNP filtering \\
\hline-0 & A unique output prefix for this run of haplotype caller \\
\hline$-\mathrm{p}$ & The number of individuals in each pool \\
\hline$-t$ & The number of threads to use during processing \\
\hline-1 & The number of times to iterate across the SNPs within each order \\
\hline$-r$ & $\begin{array}{l}\text { How high a residual should be able to exist after adding a SNP, } \\
\text { and is used to defer processing of a SNP where the residual } \\
\text { doesn't reduce enough to another iteration. }\end{array}$ \\
\hline --dropFinal & $\begin{array}{l}\text { A flag which pairs with -r to remove SNPs with a high residual } \\
\text { entirely at the end if they don't reduce the residual enough. May } \\
\text { not work with current random ordering algorithm; don't use for } \\
\text { now. }\end{array}$ \\
\hline-- genpop & A flag that instructs CallHap to generate genpop output \\
\hline --structure & $\begin{array}{l}\text { A flag that instructs CallHap to generate structure formated } \\
\text { output }\end{array}$ \\
\hline --numRandom & $\begin{array}{l}\text { Controls how many psudo-random orderings of SNPs to use, and } \\
\text { should be a value greater than zero. I set this value at } 100 \text { as a } \\
\text { compromise between run time and increased chance of finding } \\
\text { the correct solution; in practice, this value should be set based on } \\
\text { the number of starting haplotypes relative to the number of SNPs } \\
\text { present. If the number of starting haplotypes is close to the } \\
\text { number of SNPs, this value can be low; the maximum number of } \\
\text { haplotypes in the network is one more than the number of SNPs. } \\
\text { However, if the number of SNPs is greater than the number of }\end{array}$ \\
\hline
\end{tabular}




\begin{tabular}{|l|l|}
\hline & $\begin{array}{l}\text { haplotypes, more attempts may be needed to help resolve the best } \\
\text { network topology. }\end{array}$ \\
\hline-- numTopRSS & $\begin{array}{l}\text { This option just influences how many RSS values down are } \\
\text { processed for the final output solutions, and should be an integer } \\
\text { greater than zero. I set it at a value of 3 so I could examine the } \\
\text { higher RSS value solutions. }\end{array}$ \\
\hline
\end{tabular}

This program generates four to six output file per solution output (within the minimum number of RSS values):

- A NEXUS file (RunID_solNum_haps.nex) for network phylogeny creation; PopART (http://popart.otago.ac.nz/index.shtml) works fairly well. I've been using the TCS algorithm.

- A VCF file (RunID_solNum_PredFreqs.vcf) containing the estimated SNP frequencies based on the estimated haplotype frequencies, and the per-SNP average residuals in the INFO field

- A CSV file (RunID_solNum_freqs.csv) containing the per-pool haplotype frequencies and RSS values for each pool.

- A CSV file (RunID_solNum_Regression.csv) containing paired observed and predicted SNP frequencies from the Least Squared algorithm.

- (Optional): A Structure formatted file (RunID_solNum_iterNum.str) containing the expanded haplotype frequencies

- (Optional): A Genpop file containing the haplotype frequencies for use in Adigenet.

In addition, outputs are generated describing the original haplotypes network (RunID_Initial.nex), the unique haplotypes network (RunID_Unique.nex), raw topologies observed from each random order (RunID_RAW.csv), the frequency of each unique topology generated (RunID_topologies.csv), the frequency of occurrence for each haplotype found in any random order (RunID_summary.csv).

In terms of population-genetics analysis, haplotypes should be treated as independent alleles at a single locus. 


\section{Common Errors:}

\begin{tabular}{|l|l|}
\hline Problem & Solution \\
\hline Quick-start pipeline produces empty files & $\begin{array}{l}\text { Check that input files defined in the } \\
\text { preconfig exist }\end{array}$ \\
\hline $\begin{array}{l}\text { If one occurs more frequently than the } \\
\text { other, use that one. }\end{array}$ \\
$\begin{array}{l}\text { If both occur equally, check to see if the } \\
\text { network phylogenies for each solution } \\
\text { look the same, and if the generated } \\
\text { haplotype frequencies look the same. If } \\
\text { the generated haplotype frequencies are } \\
\text { identical, it doesn't matter which } \\
\text { haplotype is actually present. }\end{array}$ \\
$\begin{array}{l}\text { If generated haplotype frequencies differ, } \\
\text { create non-biologically relevant pools } \\
\text { containing the same DNA samples, but } \\
\text { shuffled in new ways (perhaps by using } \\
\text { individual 1 from each population as one } \\
\text { pool, individual 2 from each population as } \\
\text { a second, and so on). }\end{array}$ \\
\hline
\end{tabular}




\section{Appendix H: CallHap Programs}

File structure

CallHap_VCF_Filt.py

CallHap_HapCallr.py

Modules

Modules/CallHap_LeastSquares.py

Modules/General.py

Modules/IO.py

Modules/VCF_parser.py

Modules/parallel.py 


\section{CallHap_VCF_Filt.py}

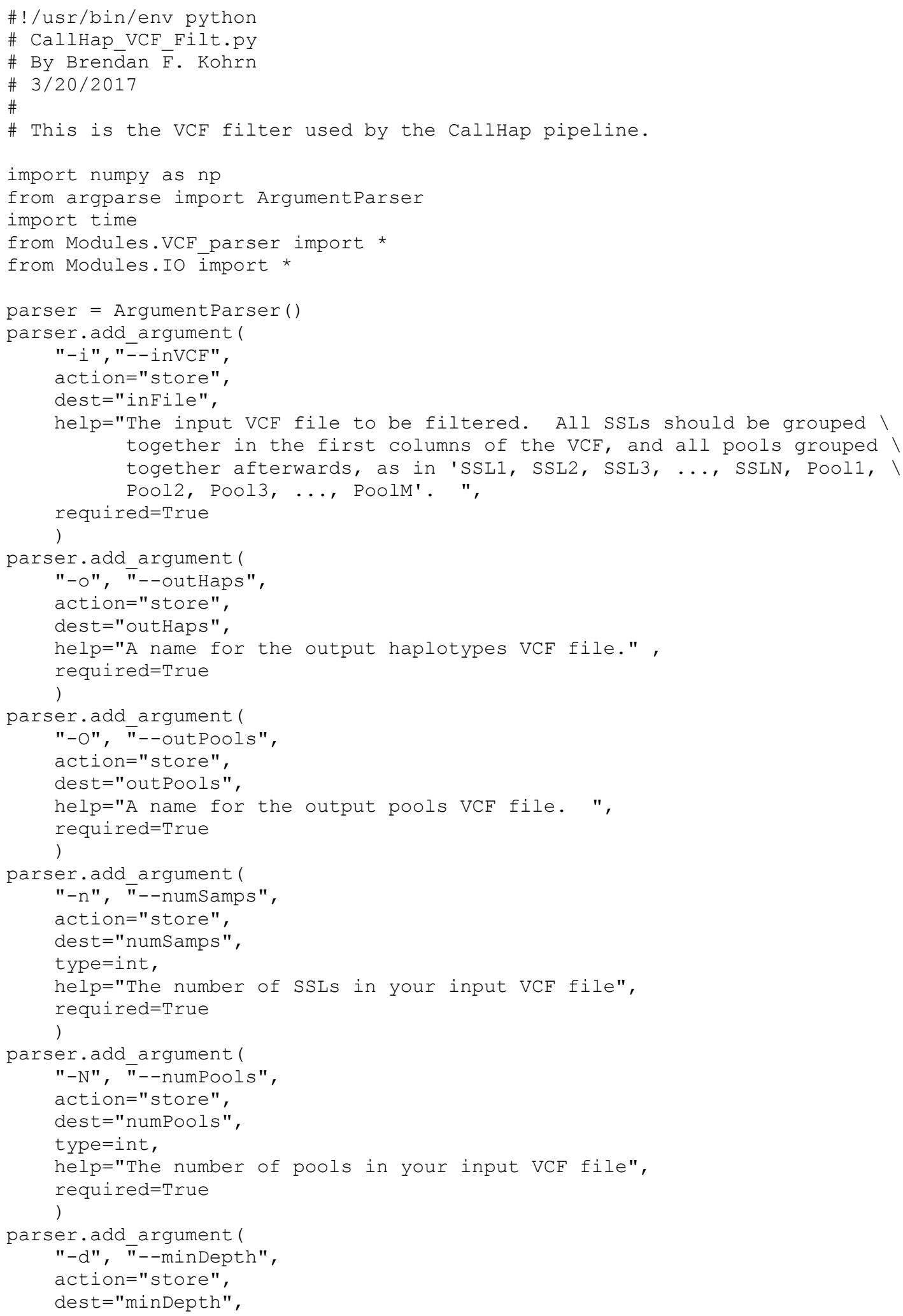




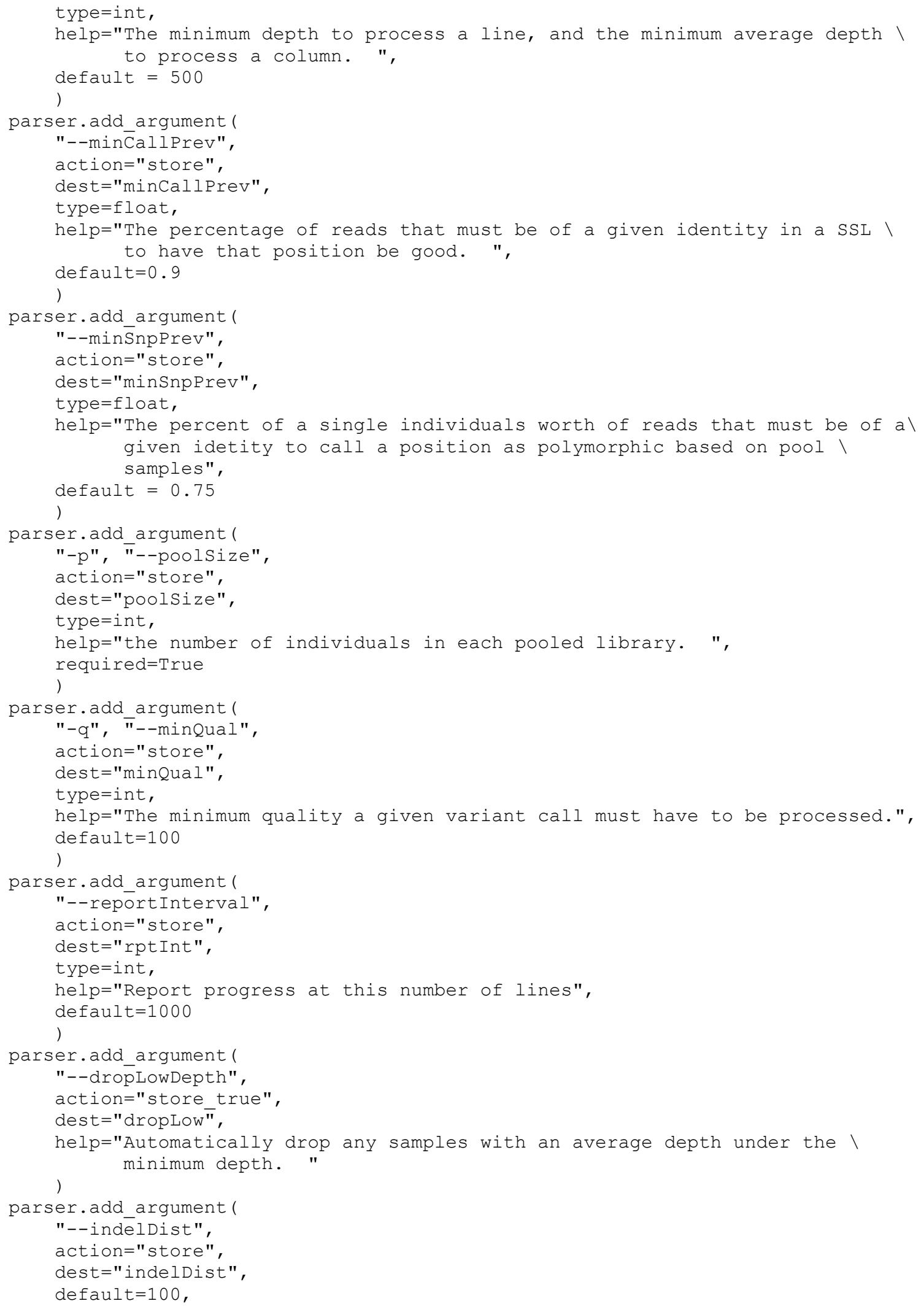




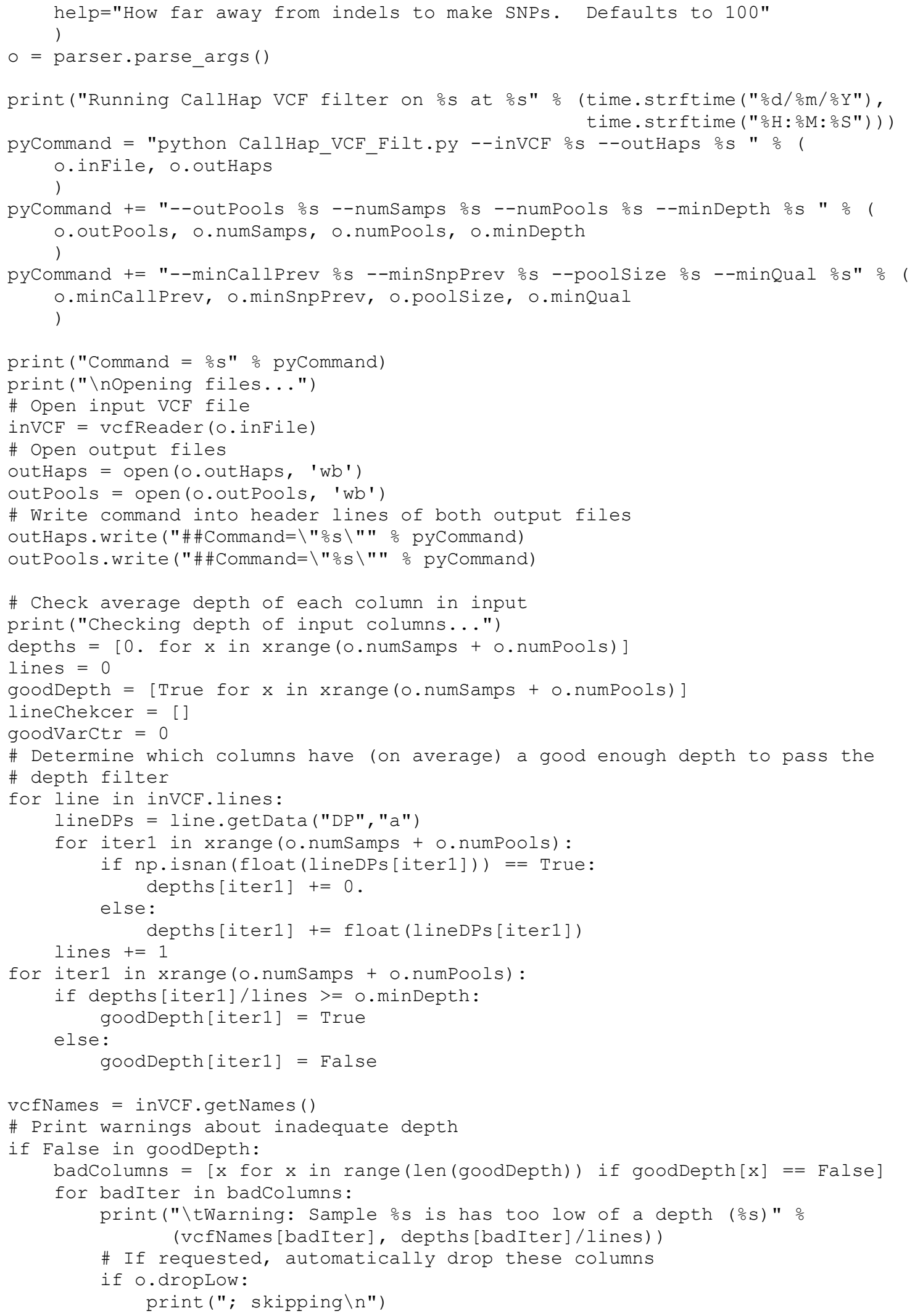




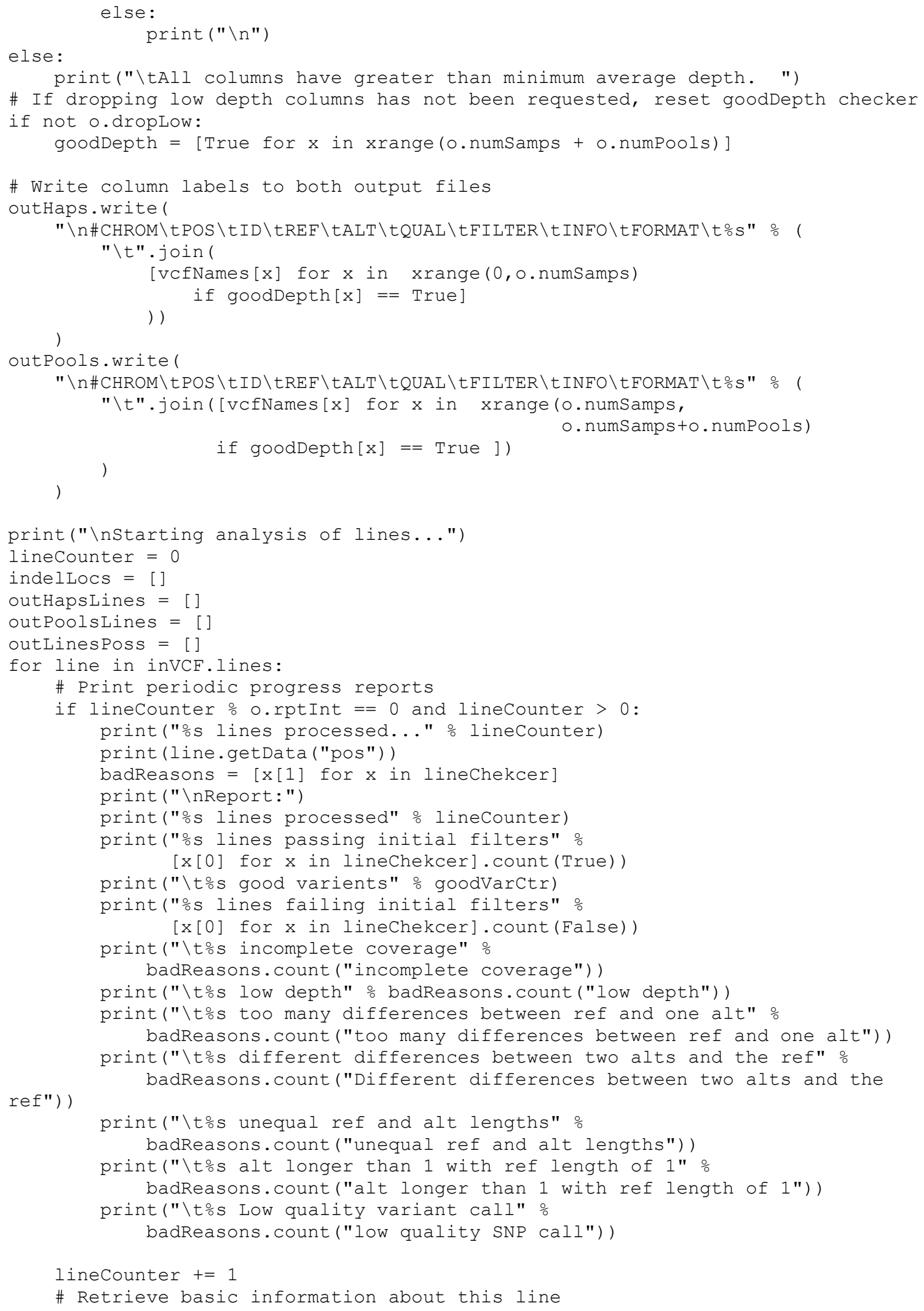




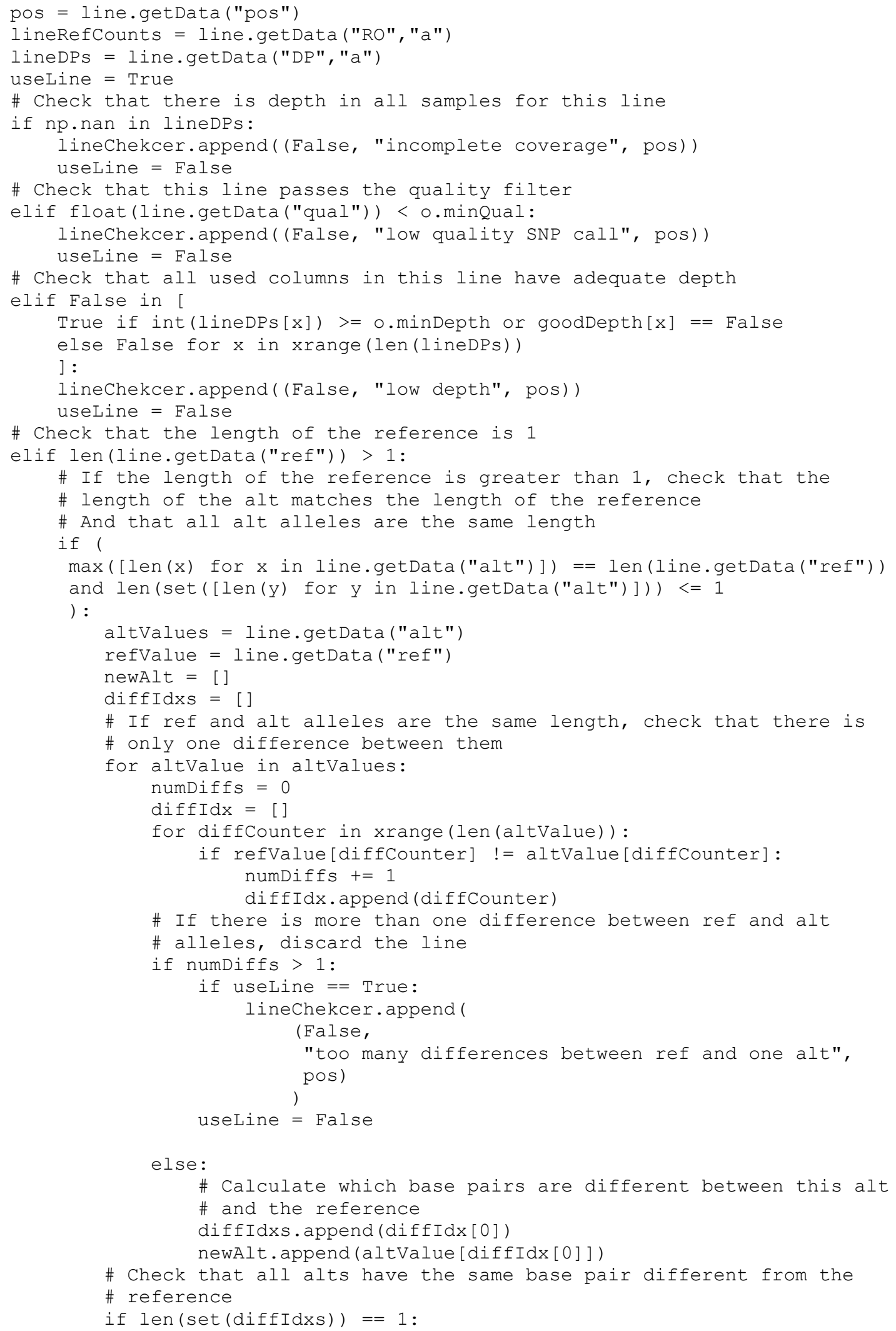




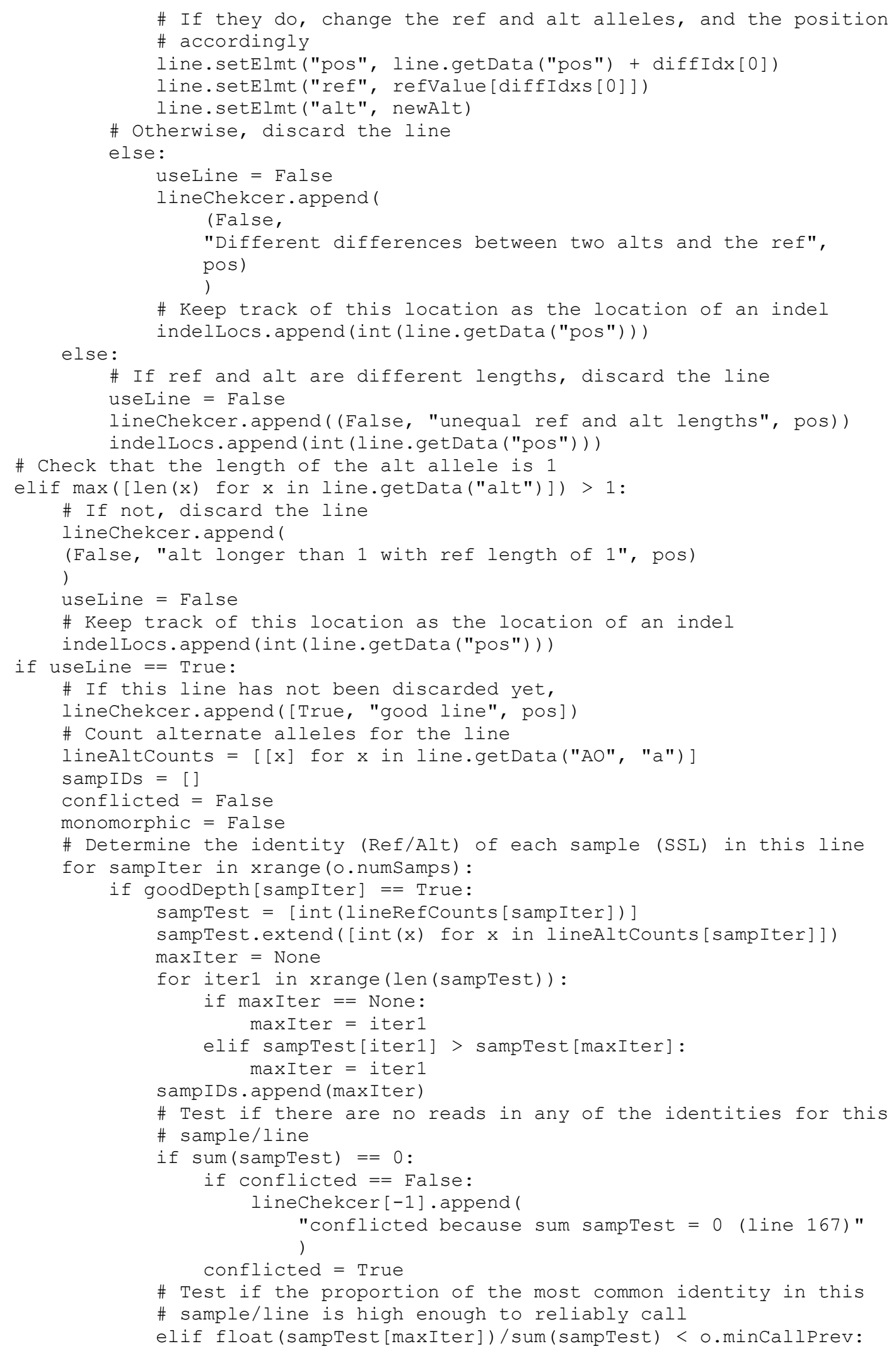




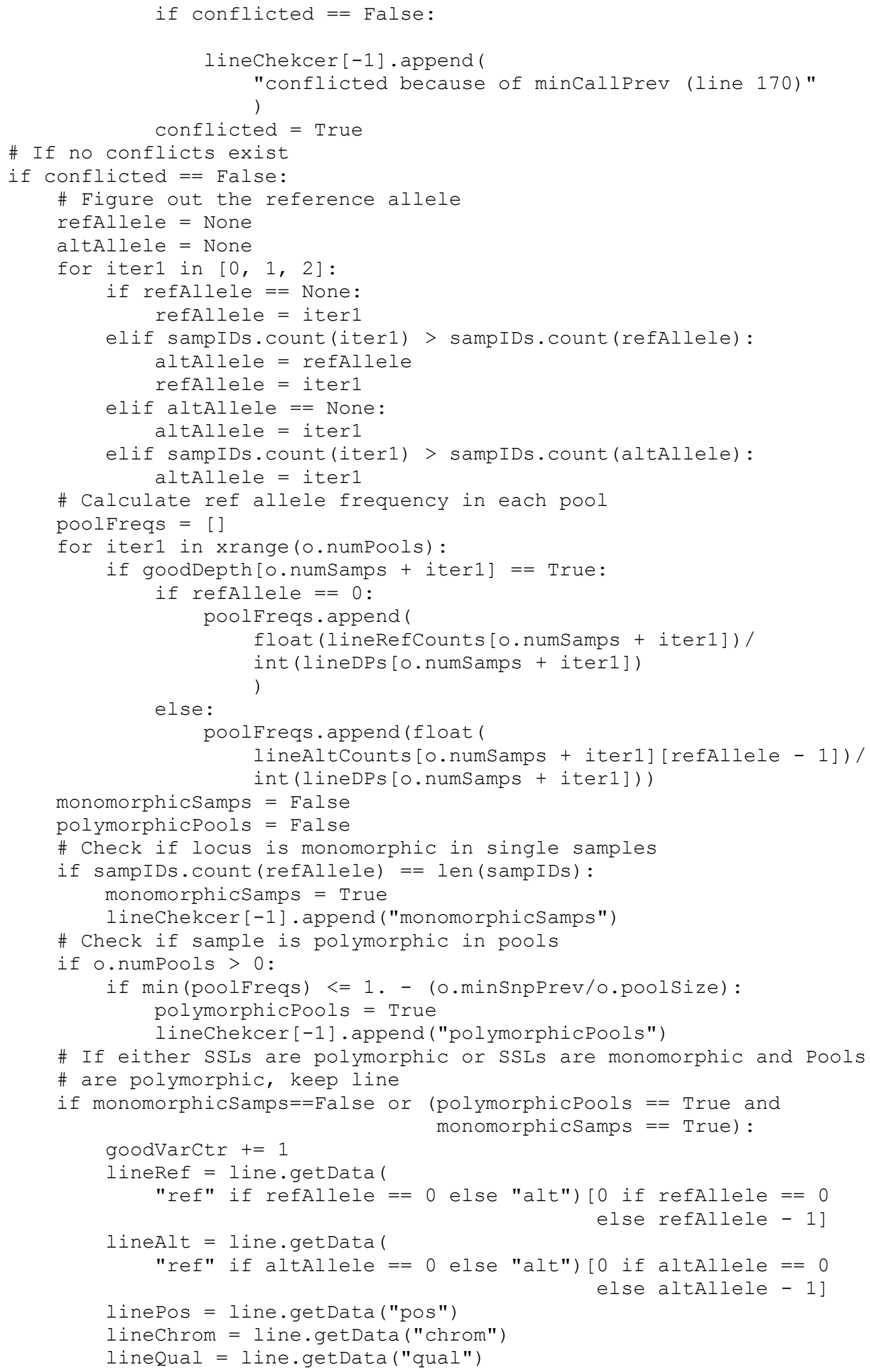




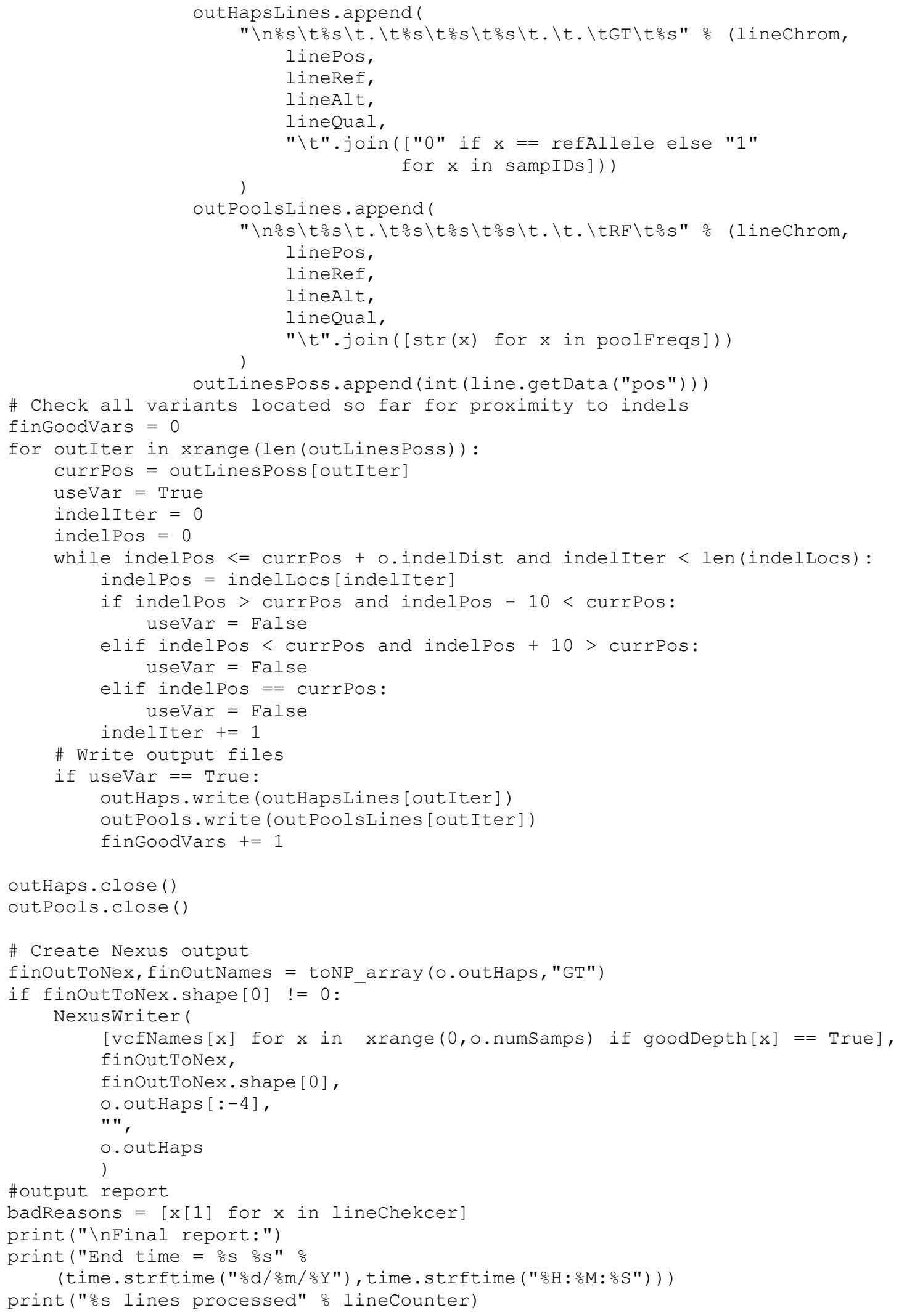




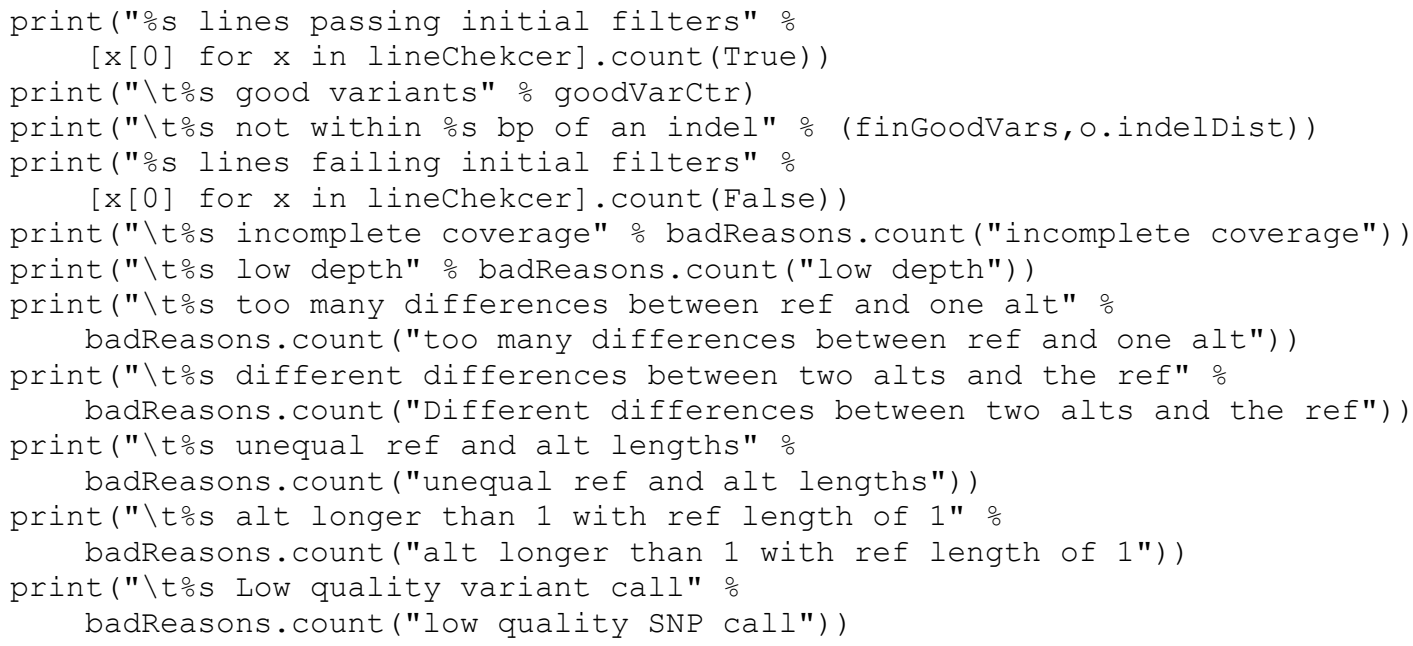




\section{CallHap_HapCallr.py}

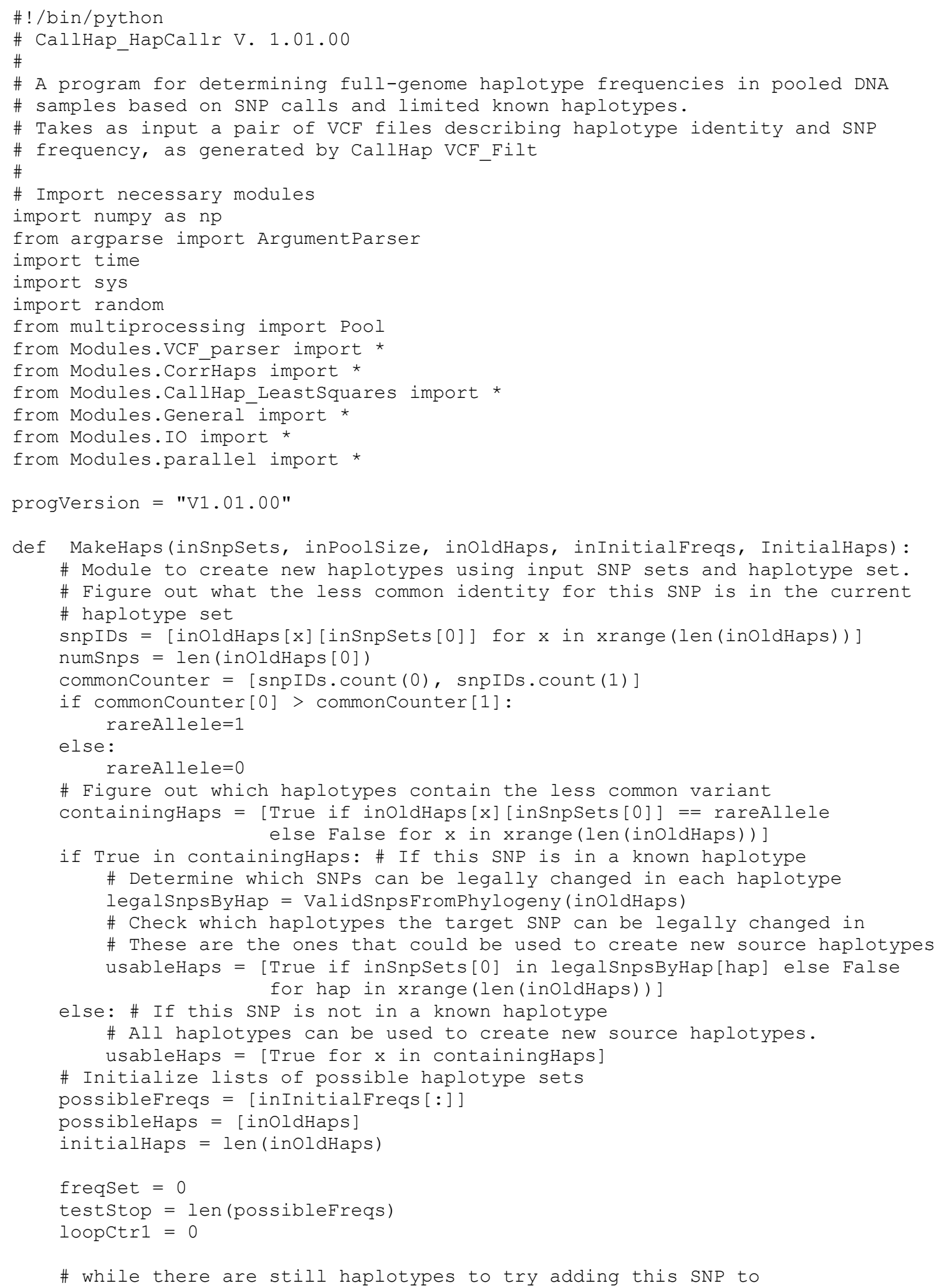




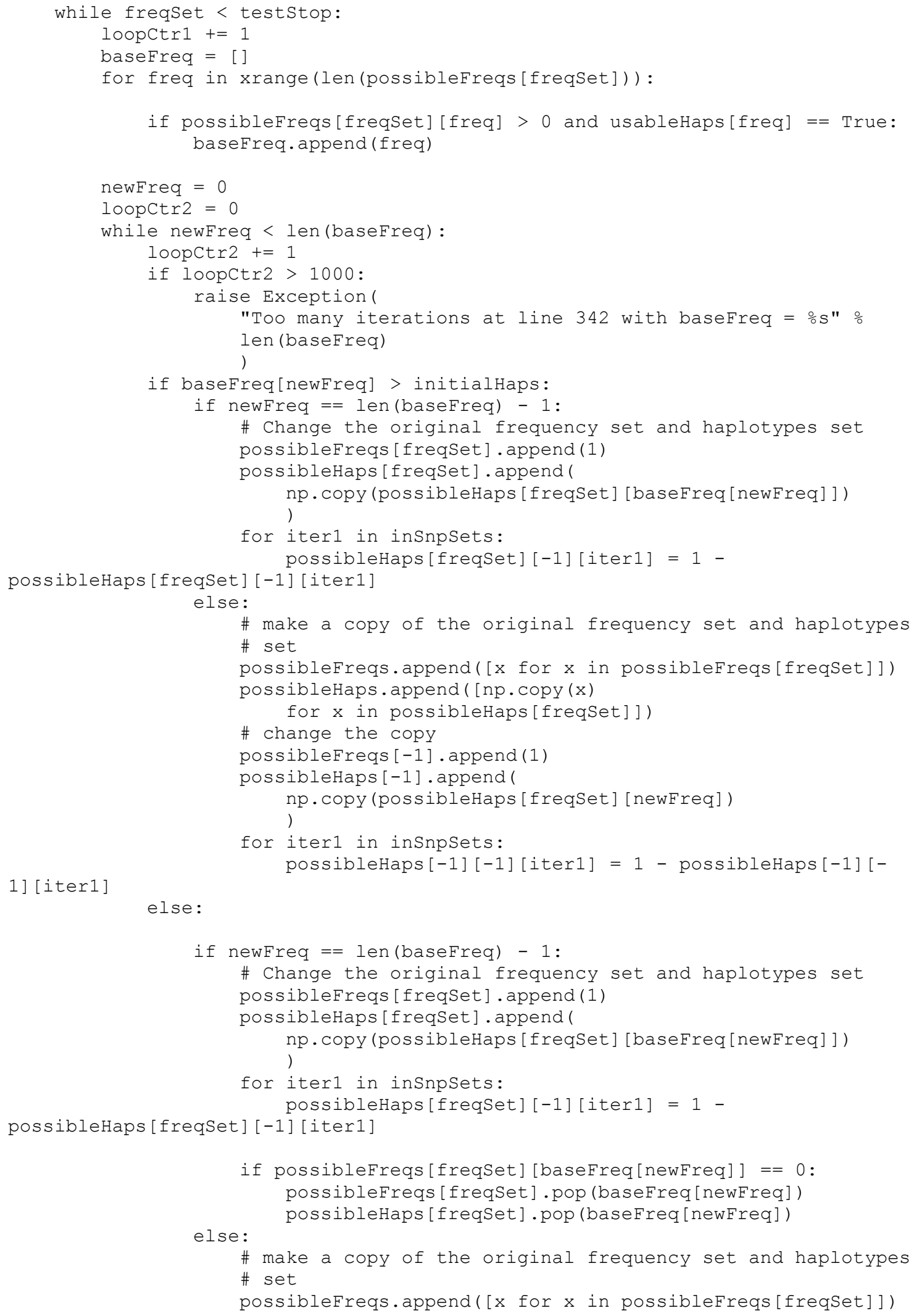


1] [iter1]

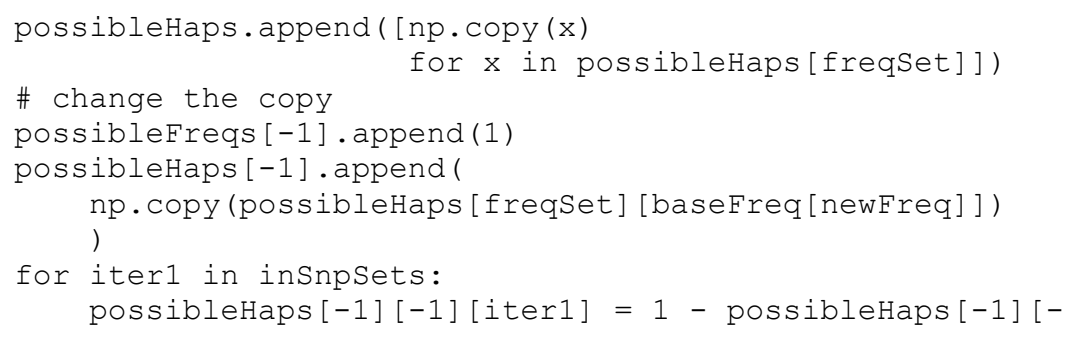

1] [iter1]

if (possibleFreqs[freqSet] [baseFreq[newFreq] ] == 0 and baseFreq[newFreq] >= InitialHaps): possibleFreqs [freqSet] . pop (baseFreq [newFreq]) 


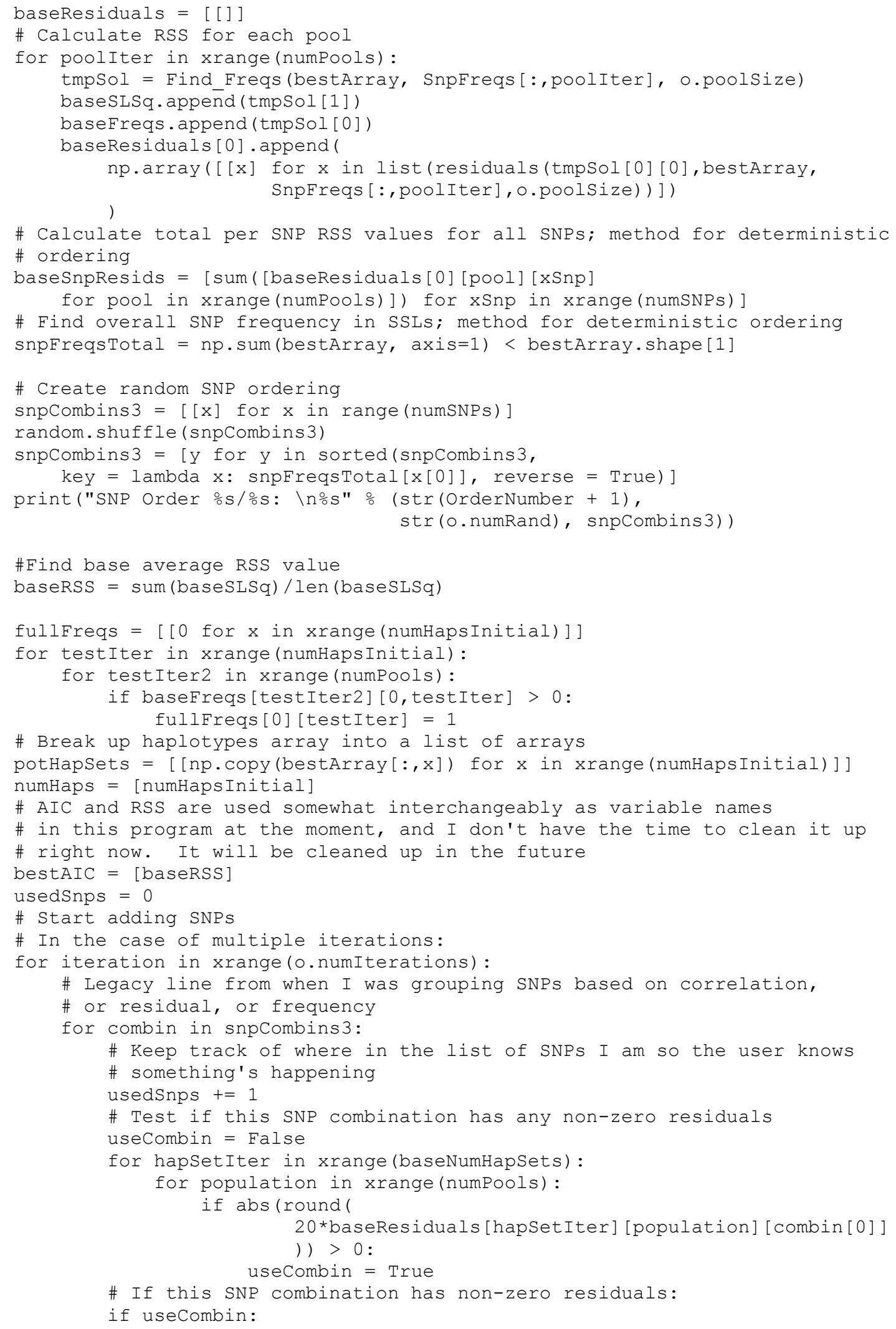




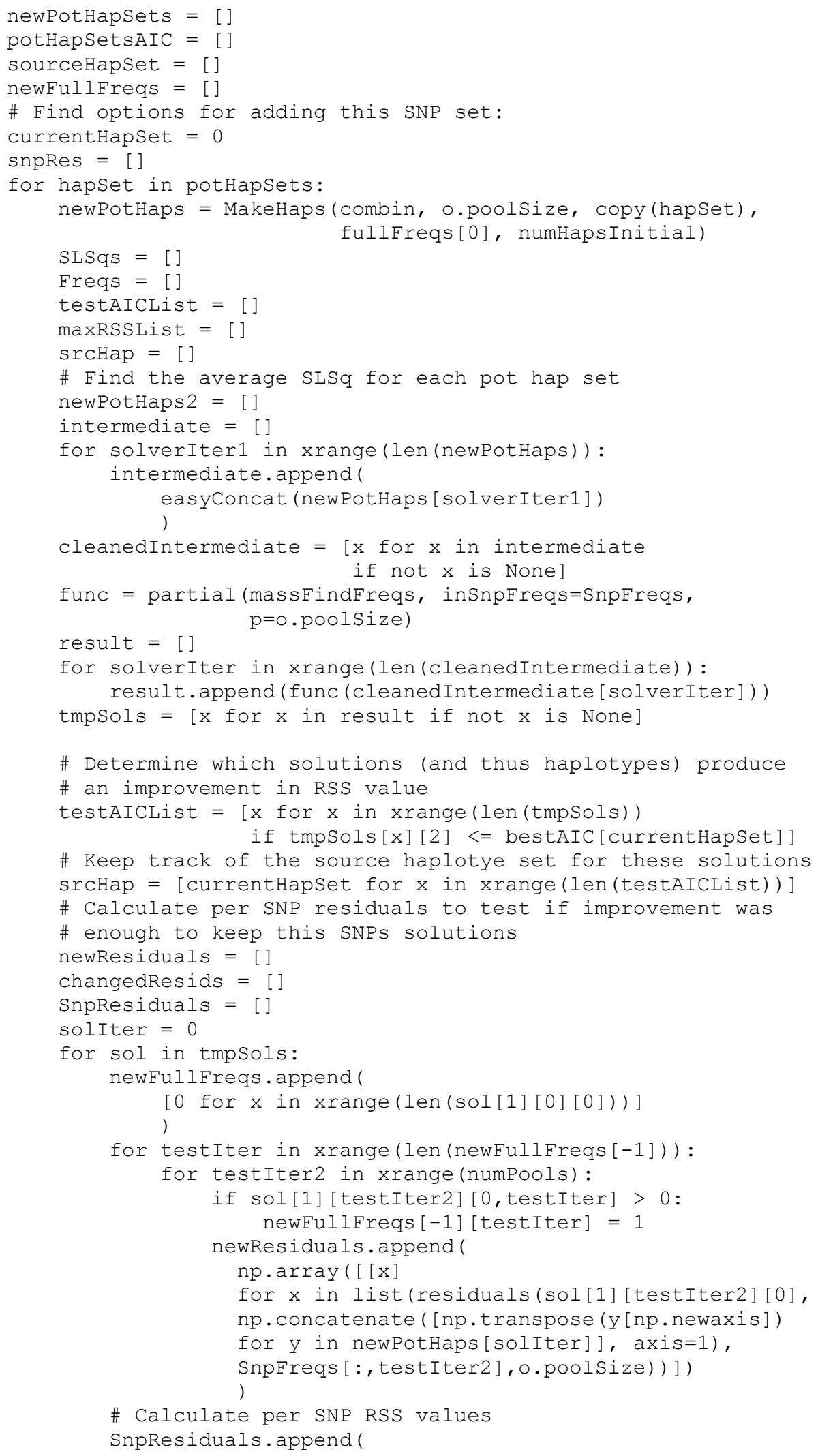




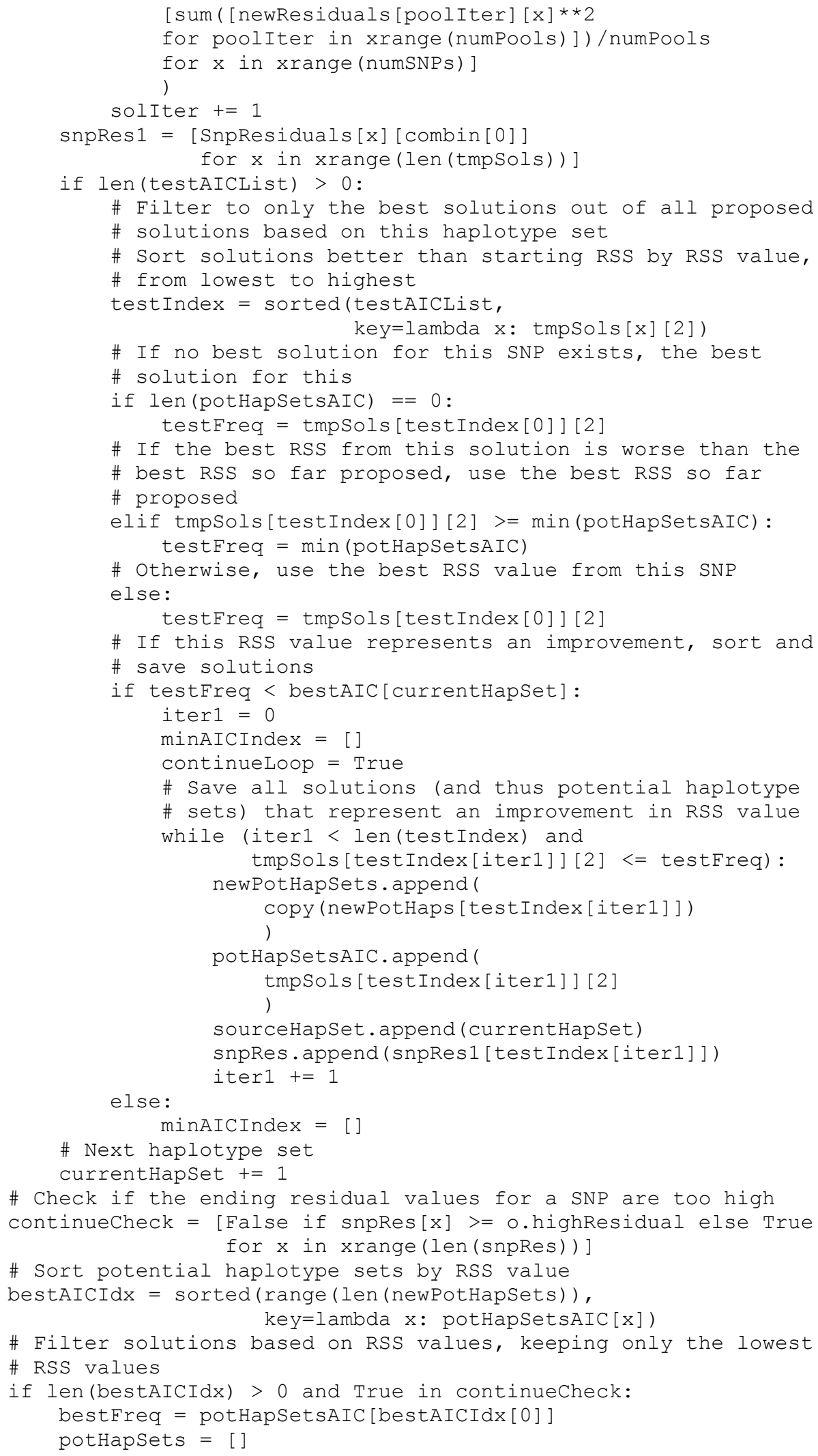




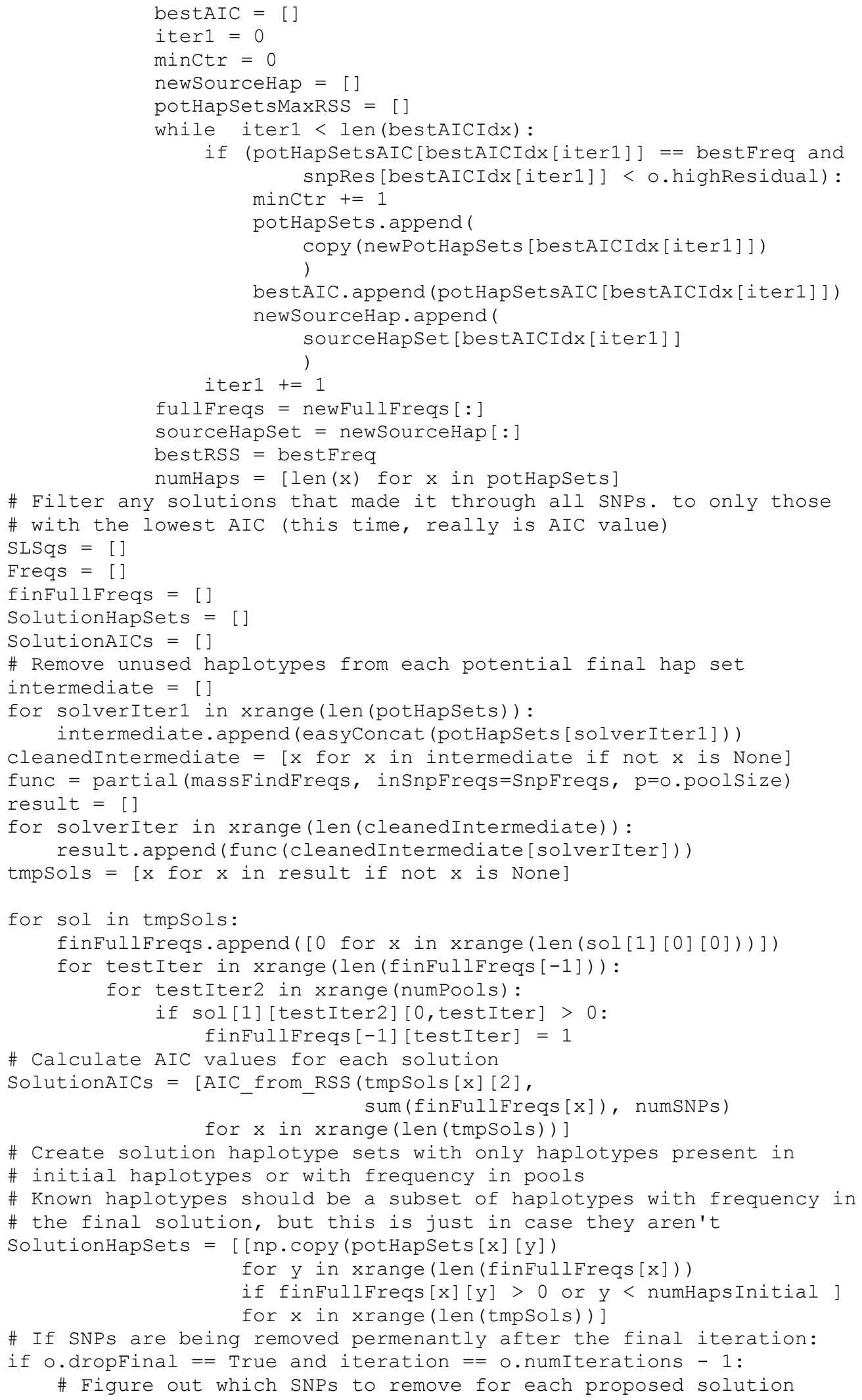




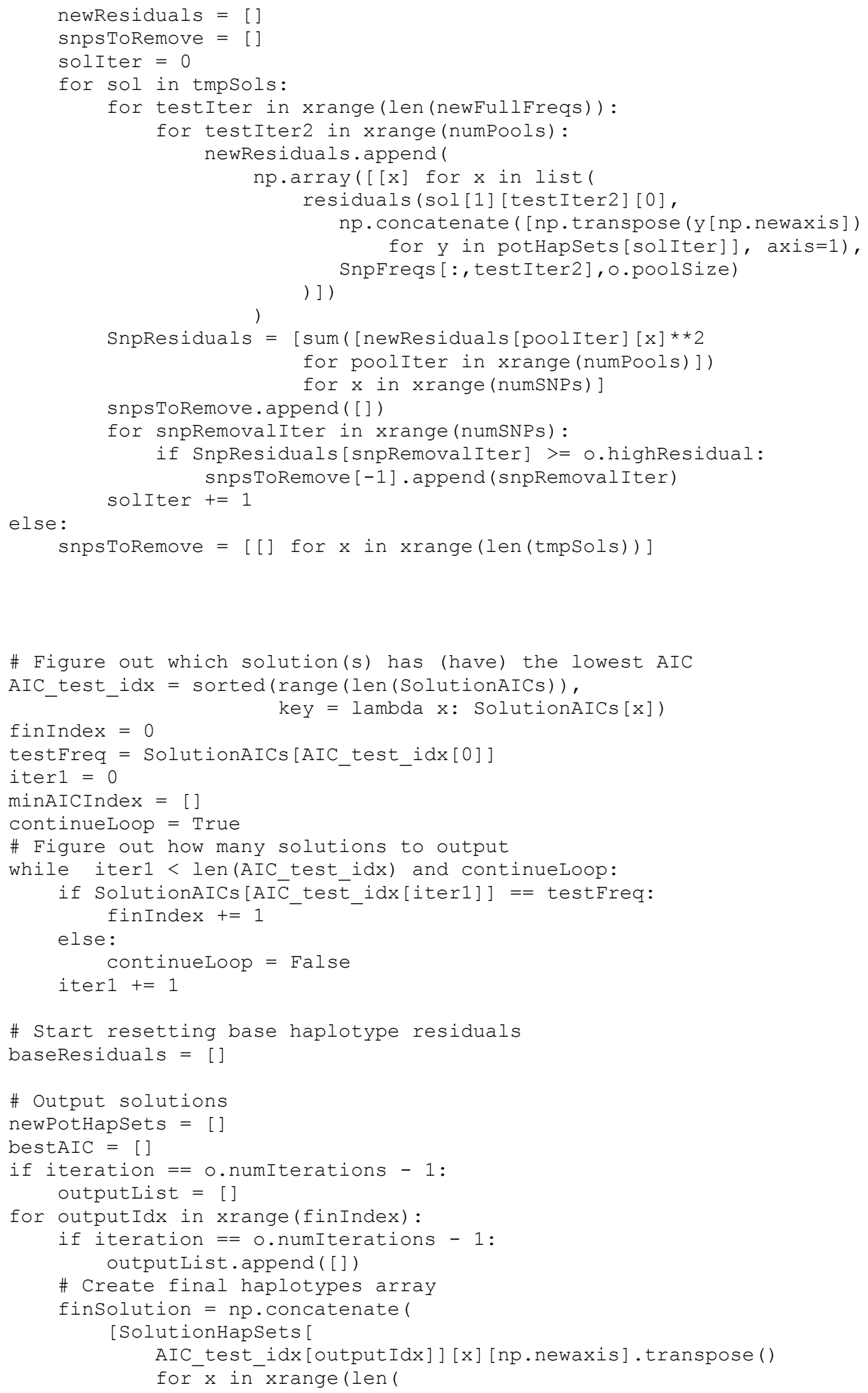




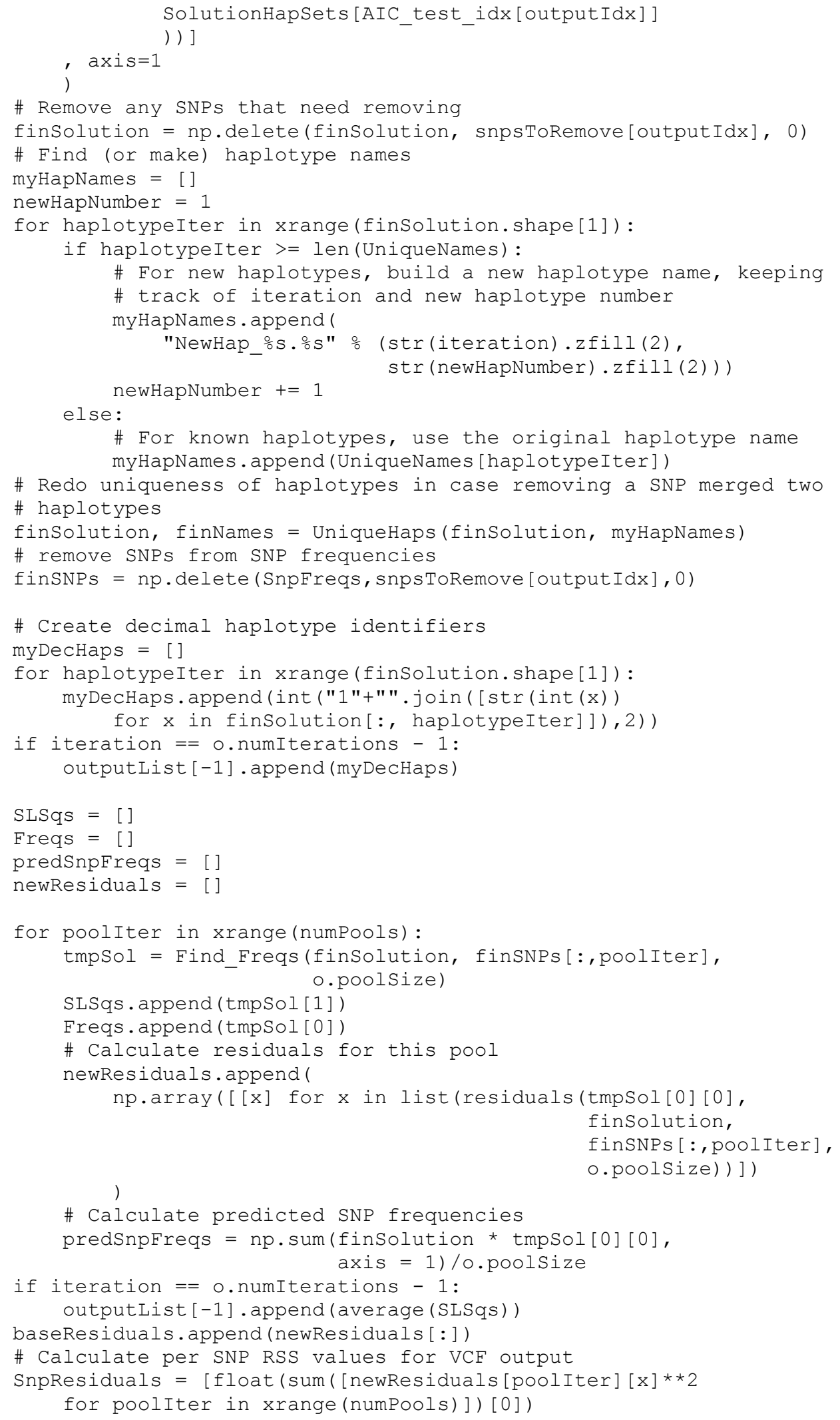


for $x$ in xrange (numSNPs-len (snpsToRemove [outputIdx])) ] bestAIC.append (sum (SLSqS) / Ien (SLSqs))

\# Save this haplotype set for the next iteration

newPotHapsets.append ( $[\mathrm{np}$.copy (finsolution $[:, \mathrm{x}]$ )

for $x$ in xrange (finsolution. shape[1])])

\# Setup for next iteration

usedSnps $=0$

numHapsInitial = len (myHapNames) \# may need some fixing

UniqueNames = myHapNames[:] \# may need some fixing

numHaps $=$ [numHapsInitial for $\mathrm{x}$ in xrange(len(newPotHapsets)) ]

outPrefix = "\%s_Iteration\%s" \% (o.outPrefix, iteration + 2)

potHapsets $=$ newPotHapsets $[:]$

fullFreqs $=[[1$ for $\mathrm{x}$ in $\mathrm{xrange}(\operatorname{len}(\operatorname{potHapSets}[\mathrm{y}]))]$

for $y$ in xrange (len (potHapsets)) ]

\# Go on to the next iteration

if iteration $=0$. numIterations - 1 :

print("Finished Random Order \%s/\%s" 은 (str(OrderNumber + 1), $\operatorname{str}(0$. numRand)) )

return (outputList)

if

\# Load options

parser $=$ ArgumentParser ()

parser.add_argument (

'-i', '--inputHaps',

action="store",

dest="knownHaps",

help = "A VCF-formatted file containing the known haplotypes encoded \ in the GT field. GT must be present in the FORMAT field, and \ ploidy must be $1 . "$ ",

required=True

)

parser.add_argument (

'-p', '--poolsize',

action="store",

type=int,

dest="poolsize",

help="The number of individuals in each pool. ",

required=True

)

parser.add_argument (

'-f', '--inputFreqs',

action="store",

dest="inFreqs",

help="A VCF-formatted file containing the input pool frequencies I encoded in the RF field. RF must be present in the FORMAT । field. ",

required=True

)

parser.add_argument (

'-o', '--outPrefix',

action="store",

dest="outPrefix",

required=True,

help="A prefix for output file names. "

)

parser.add argument (

"-v", "--version",

action="store_true",

dest="v", 


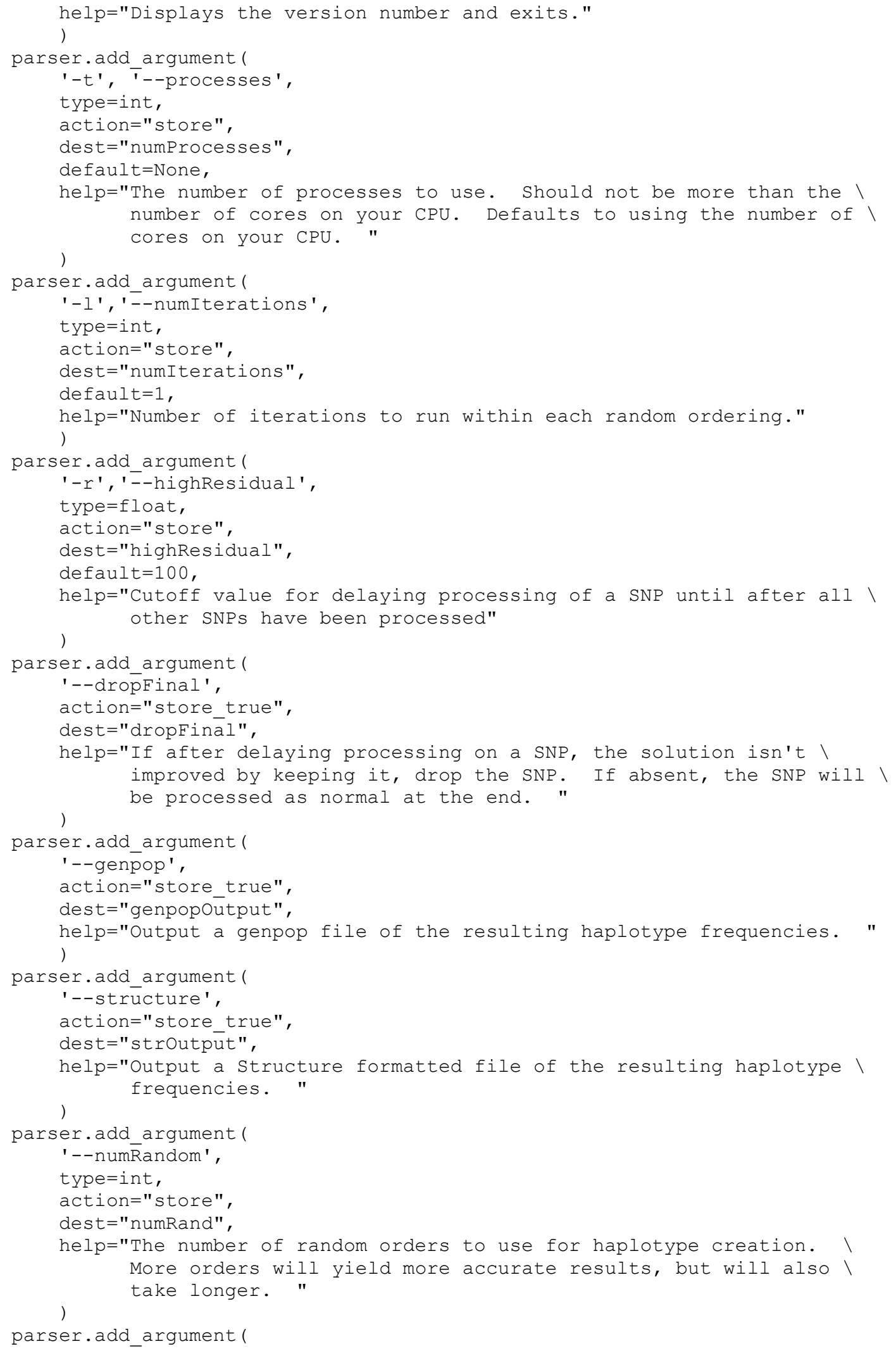




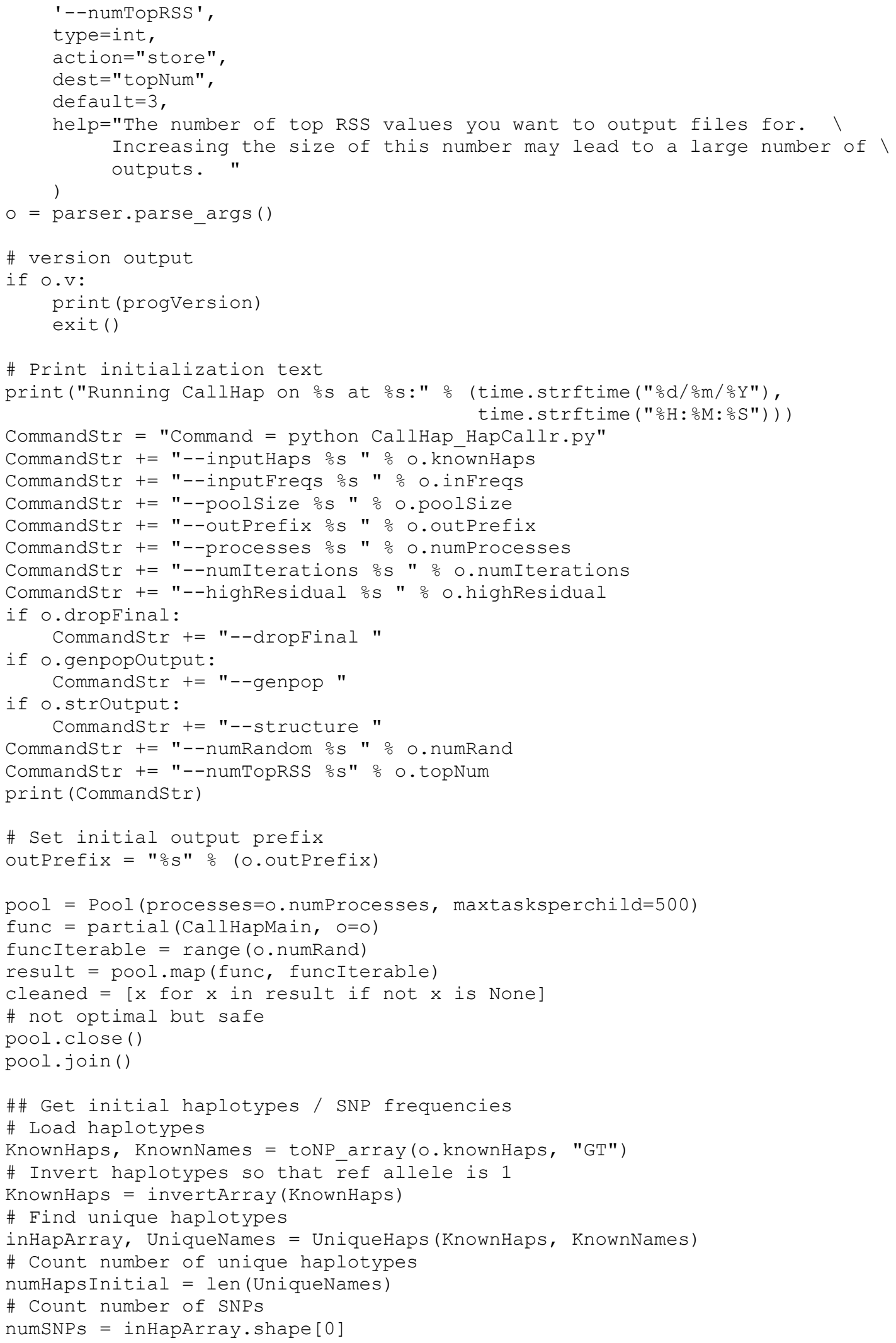




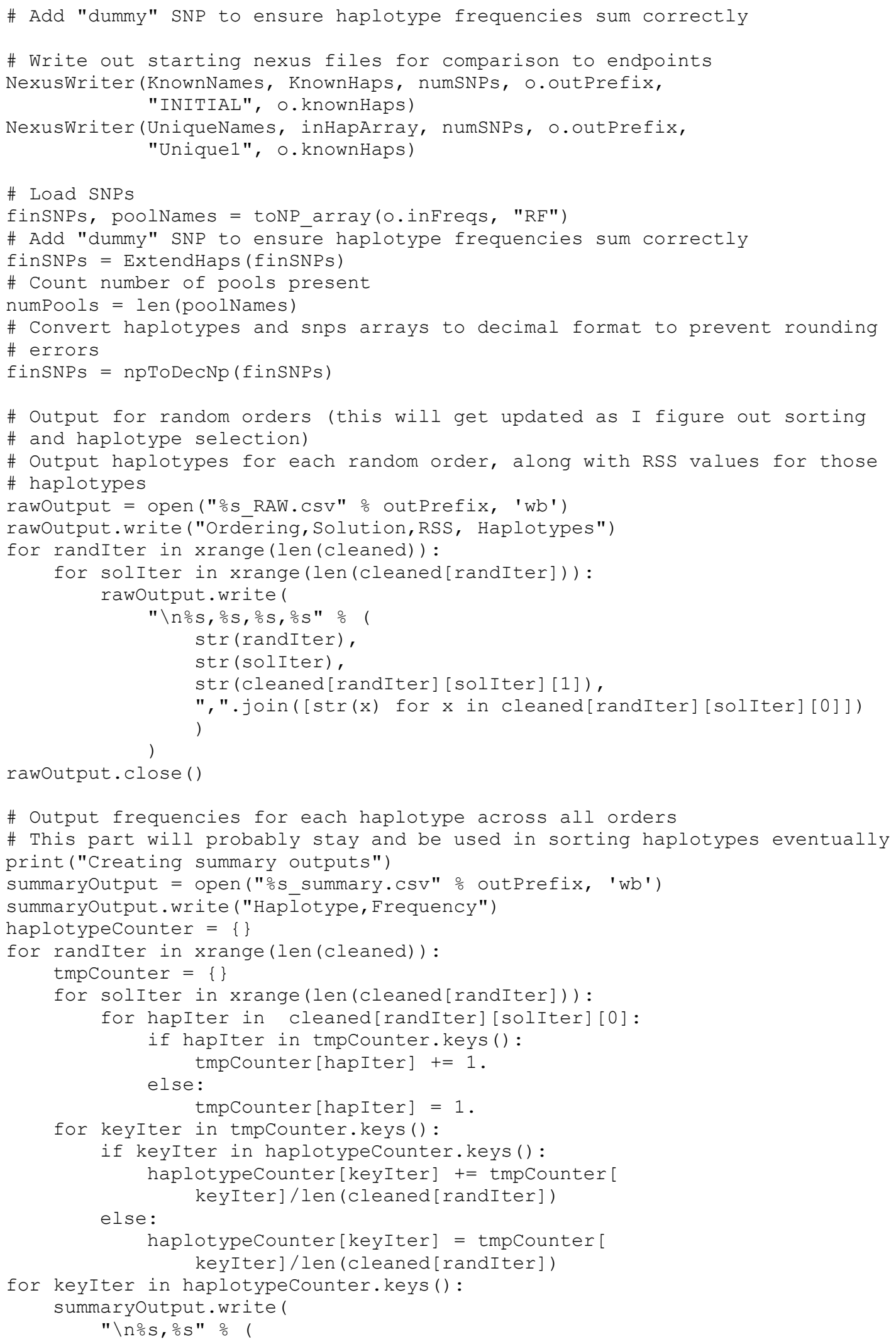




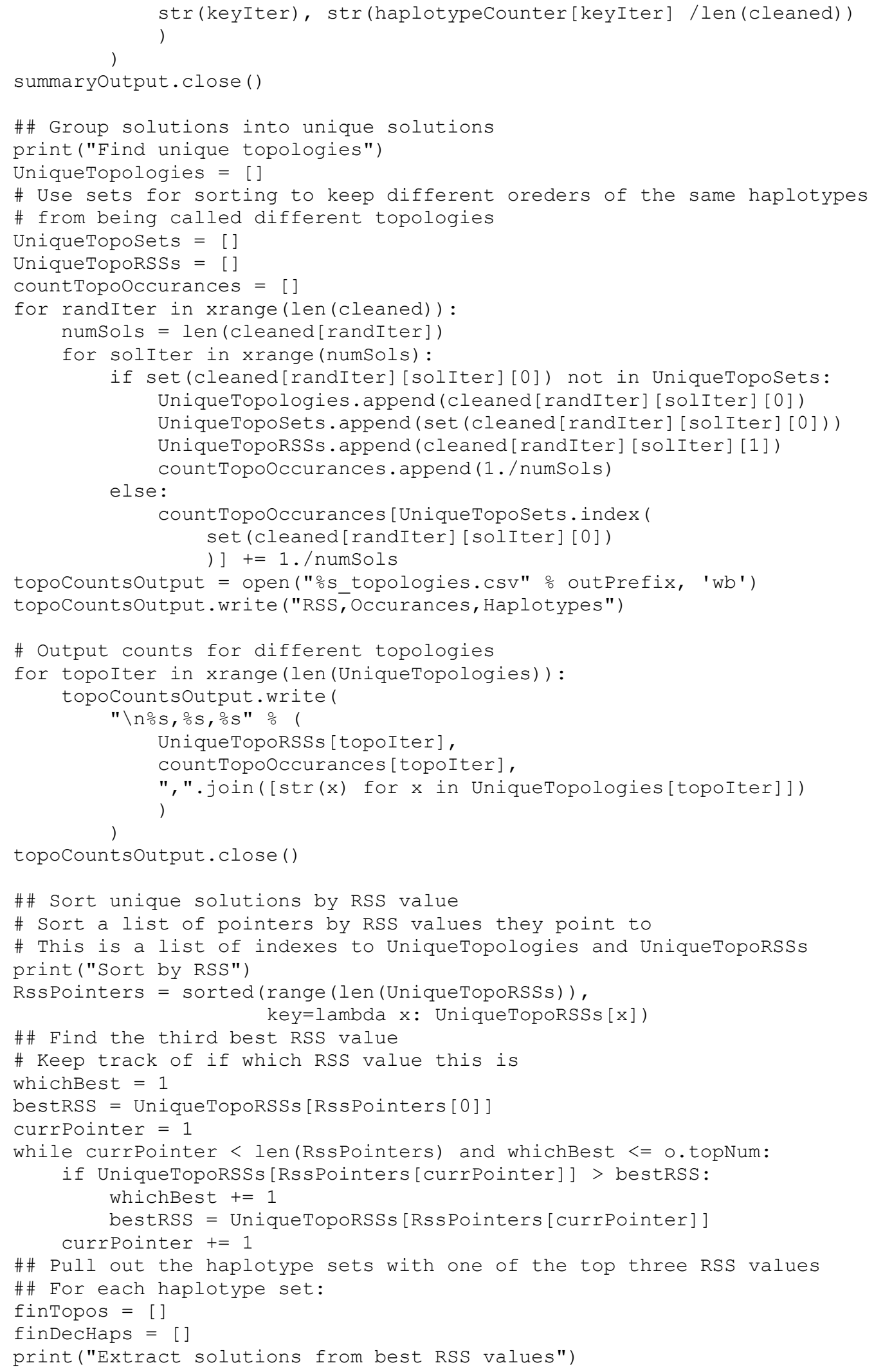




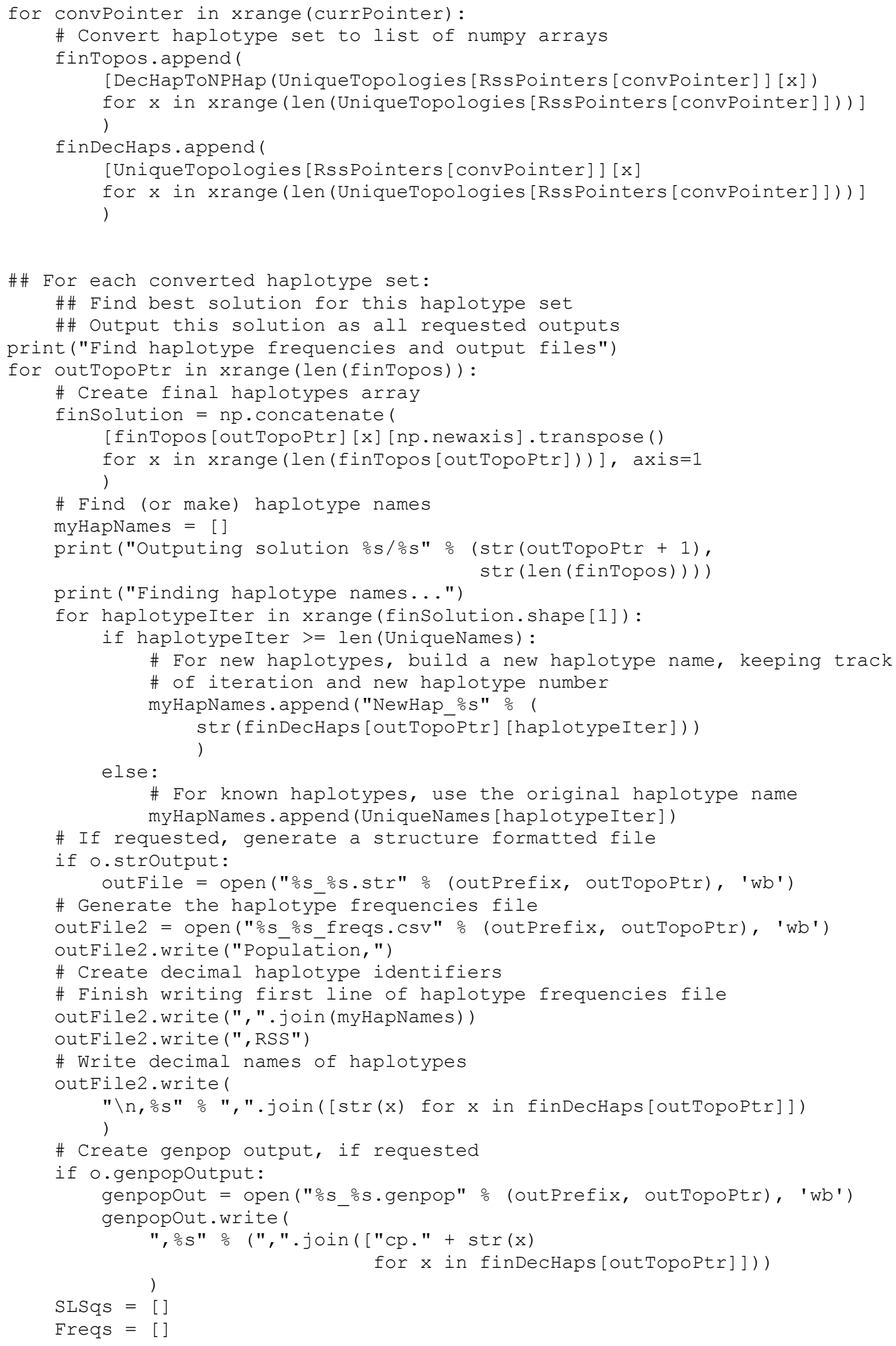




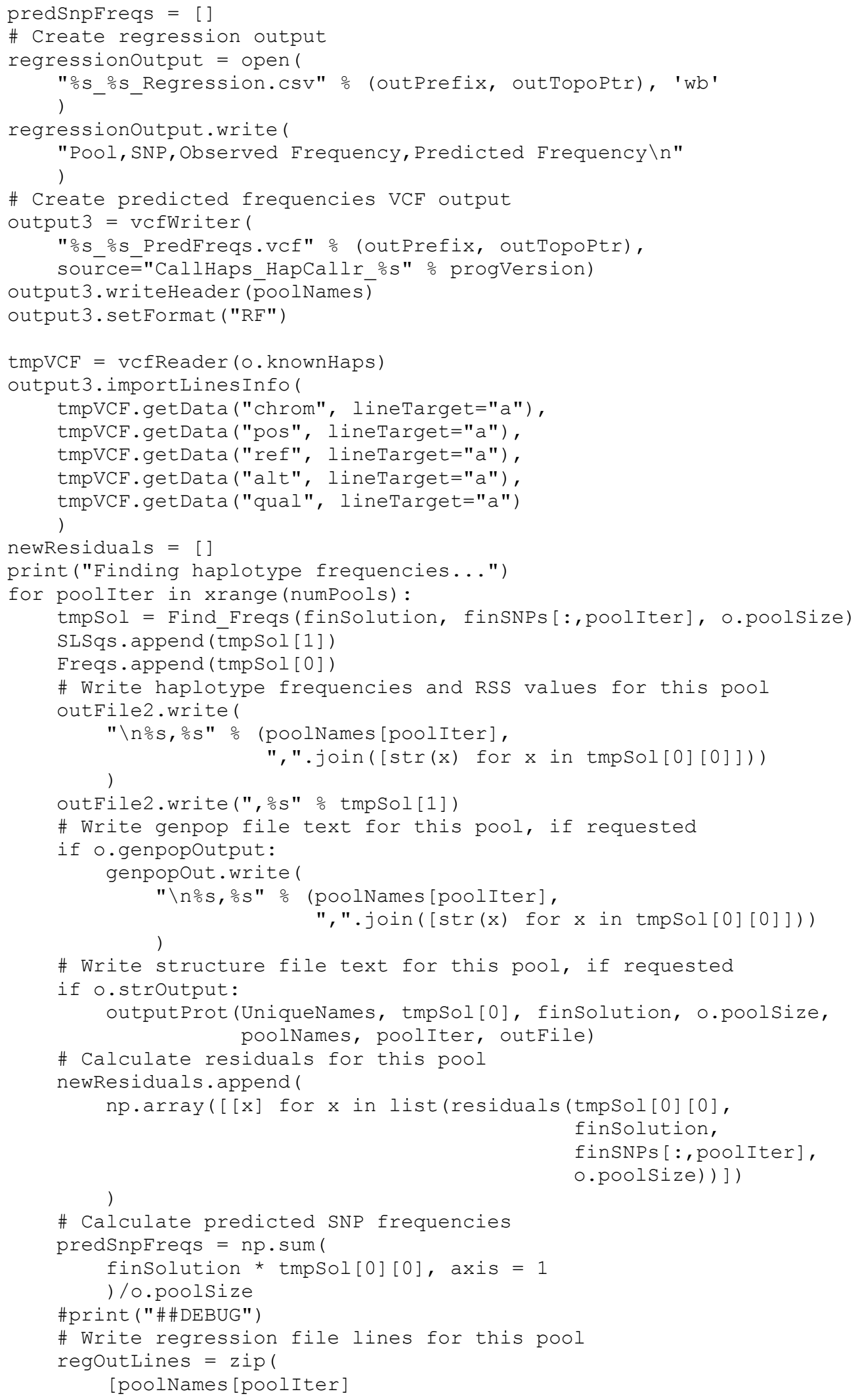




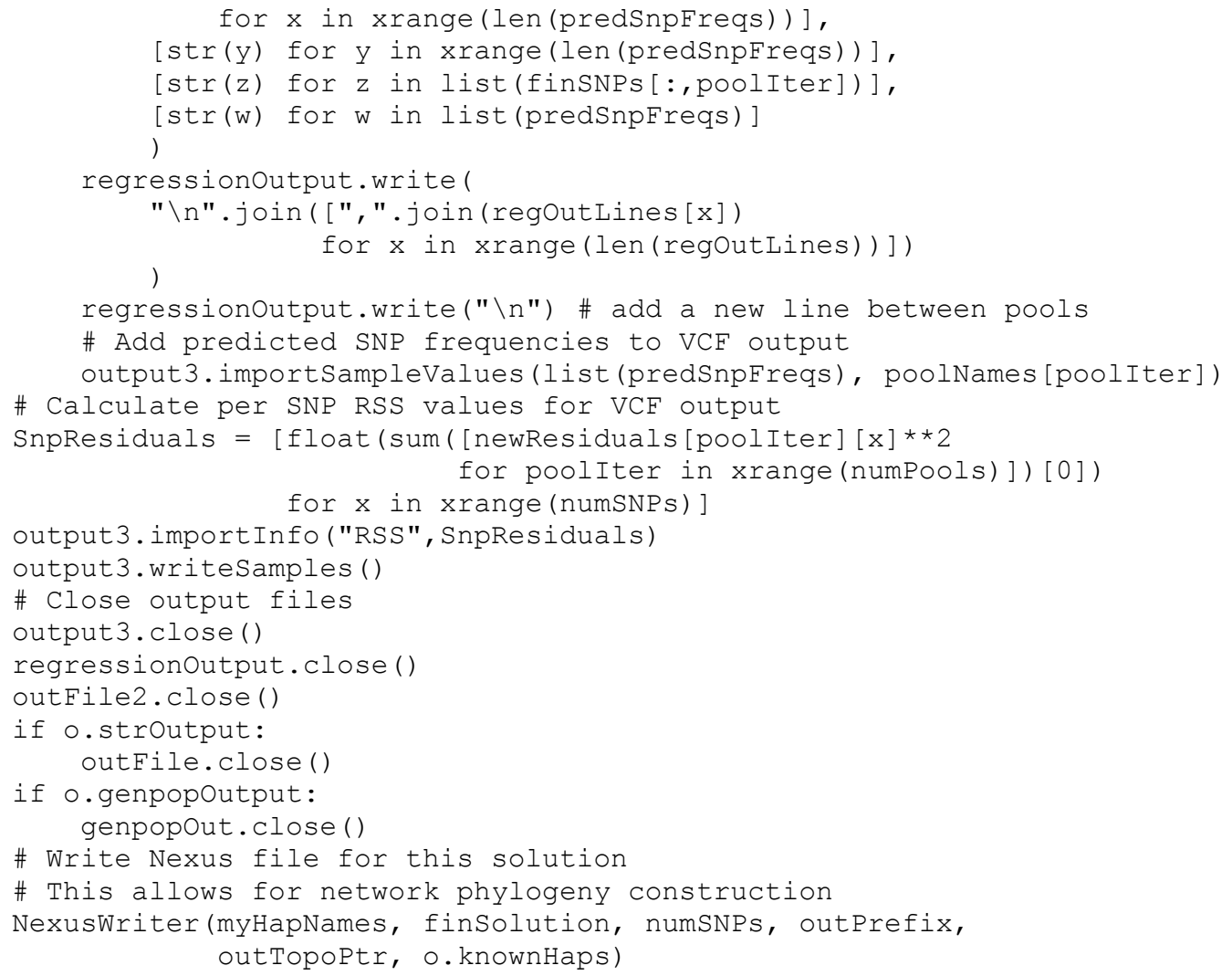




\section{Modules/CallHap_LeastSquares.py}

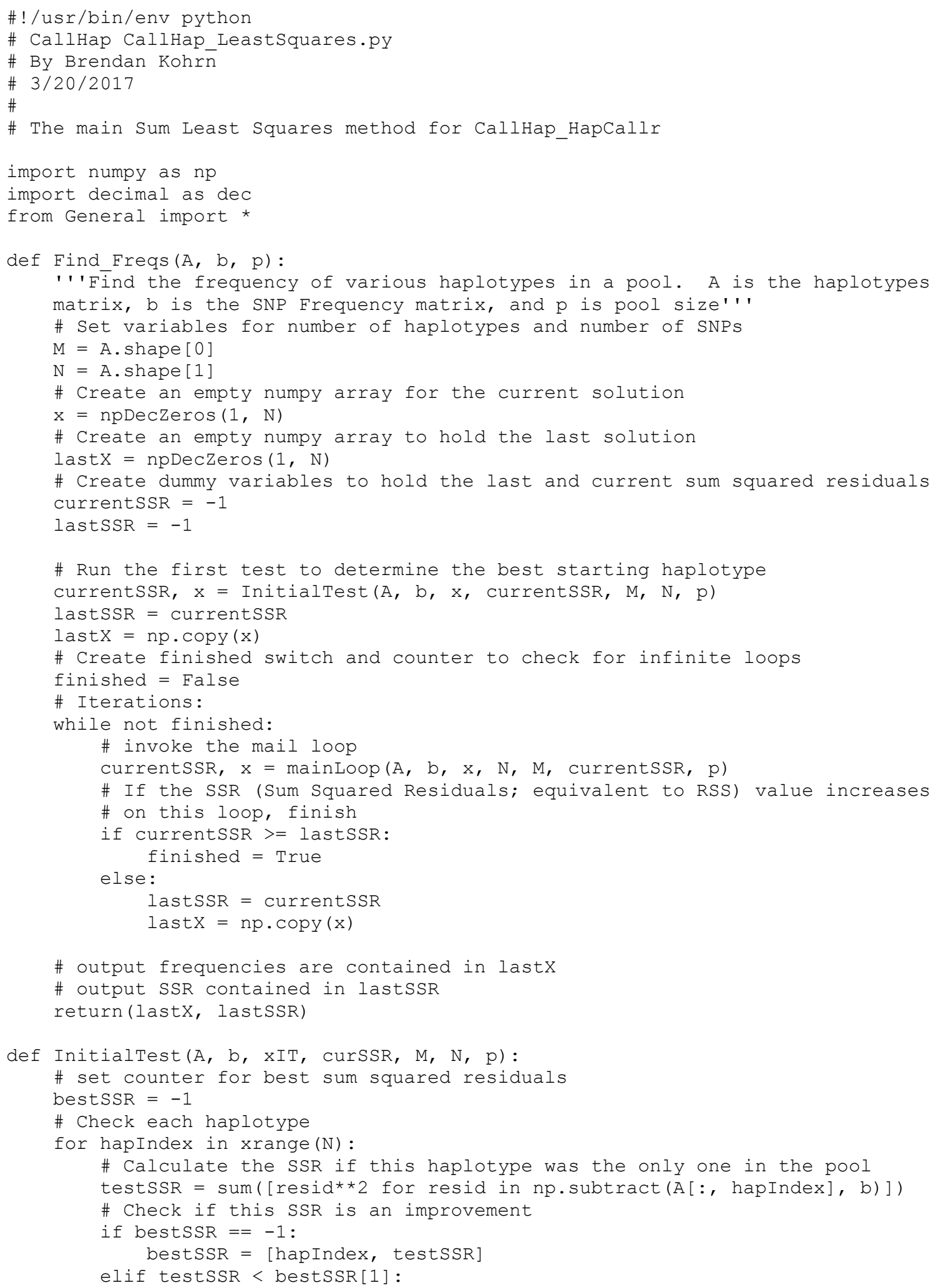




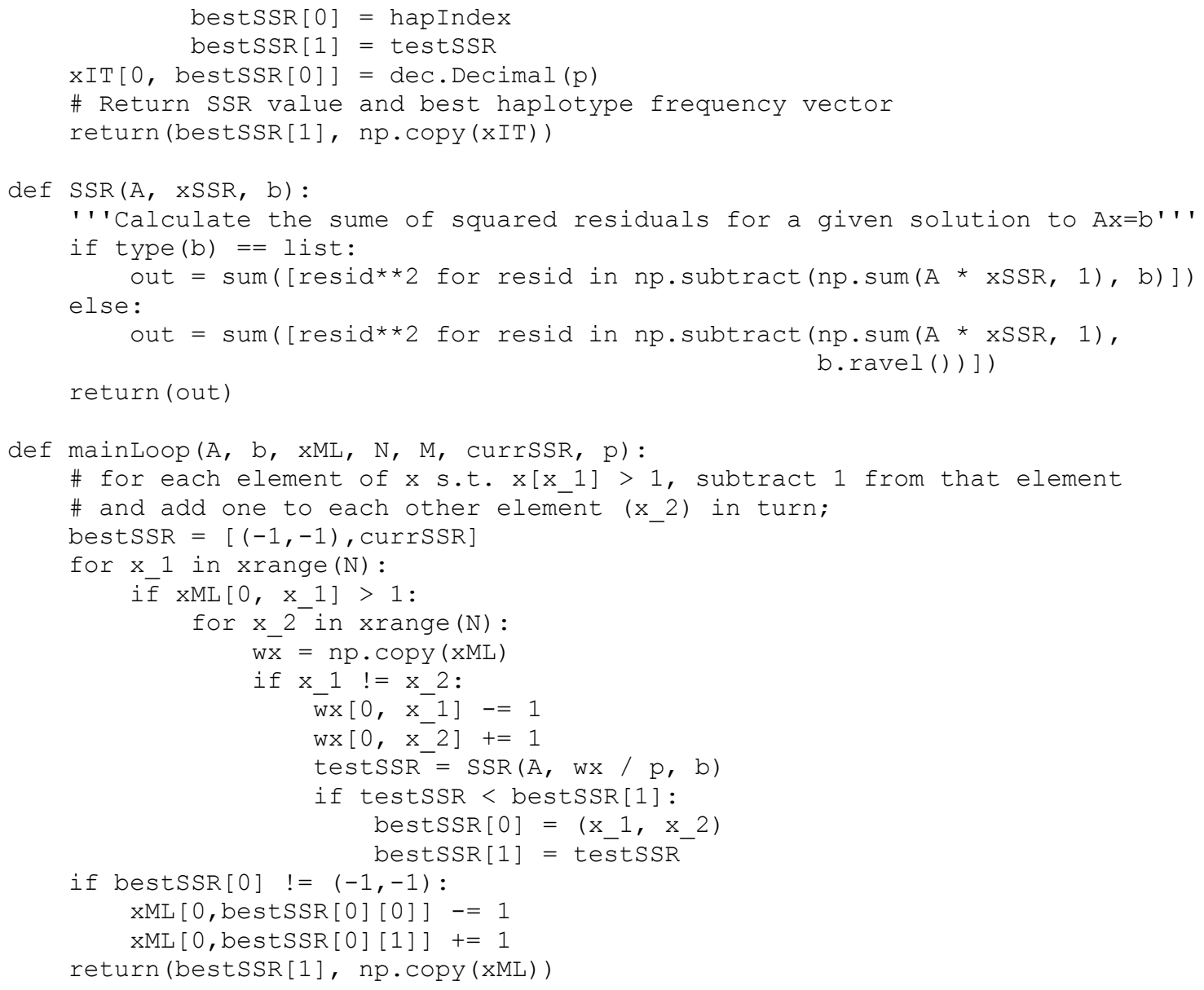




\section{Modules/General.py}

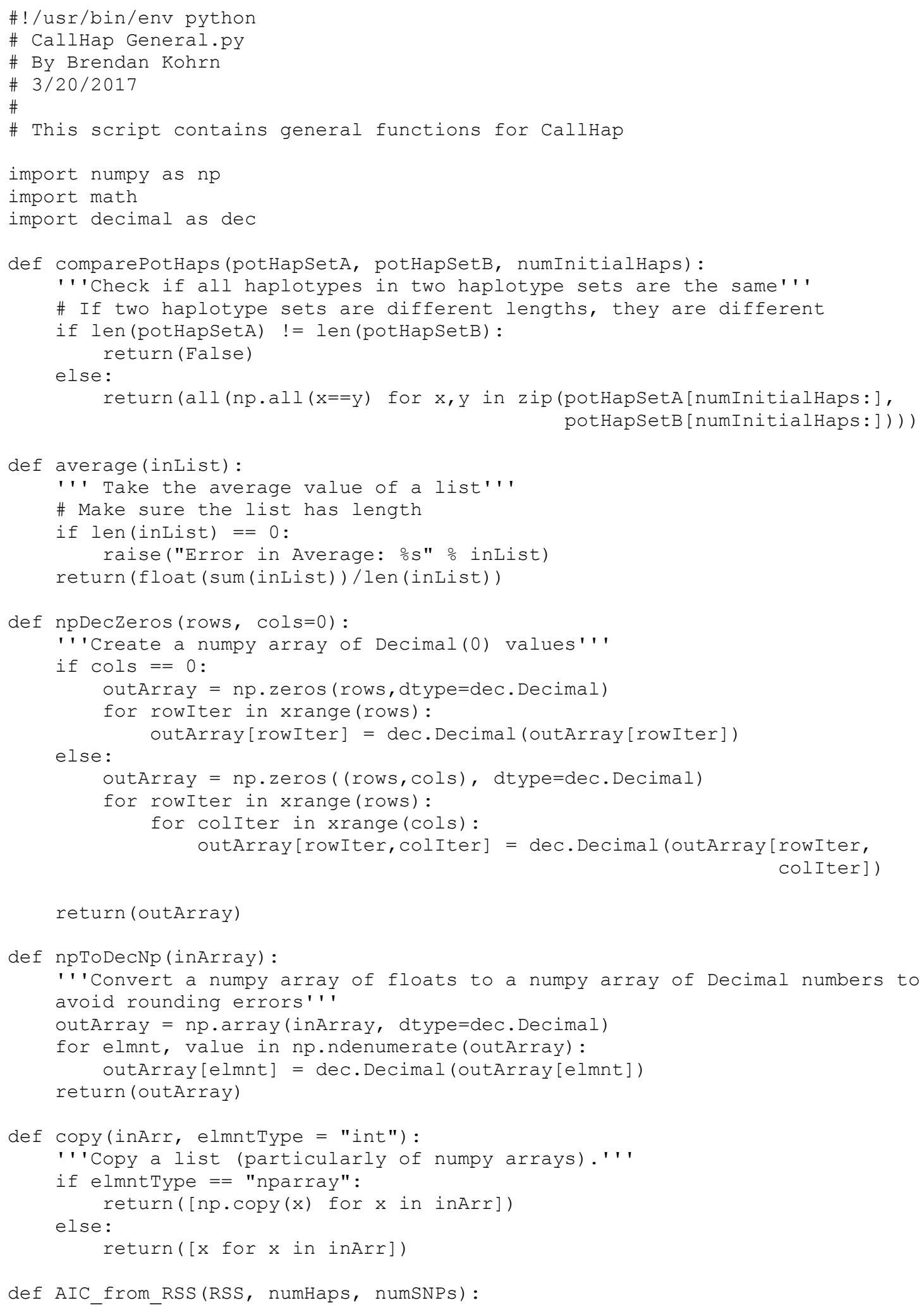




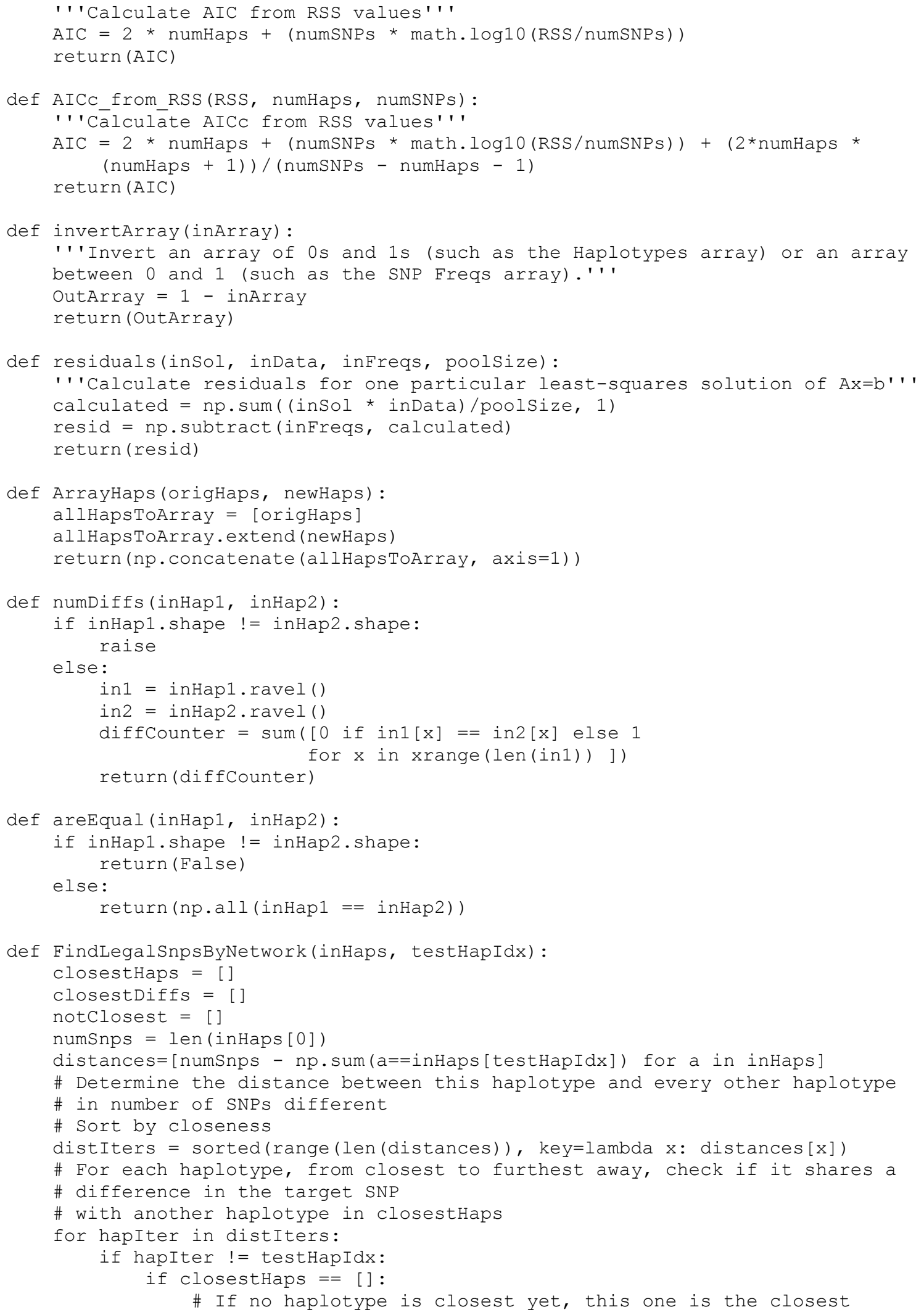




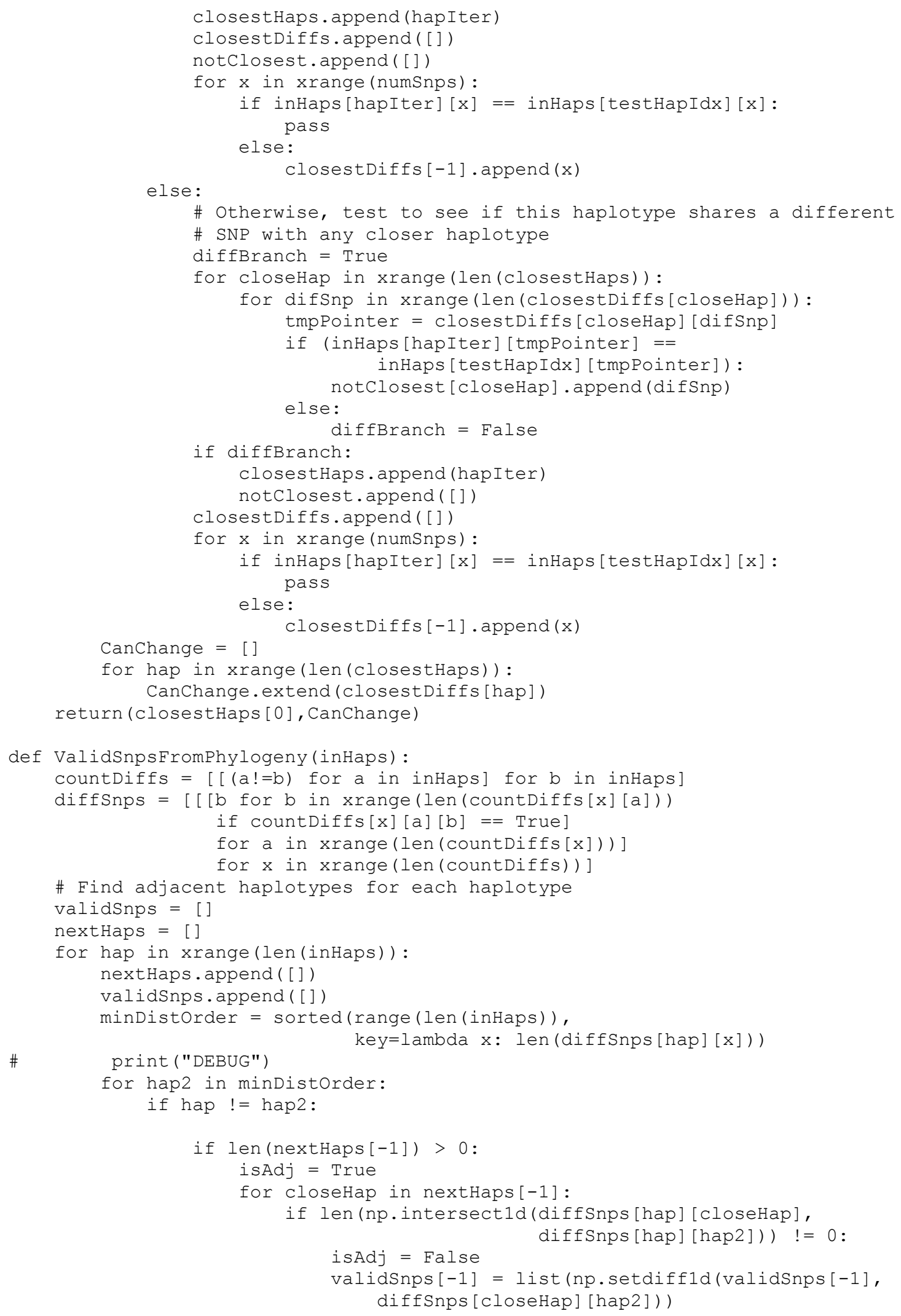




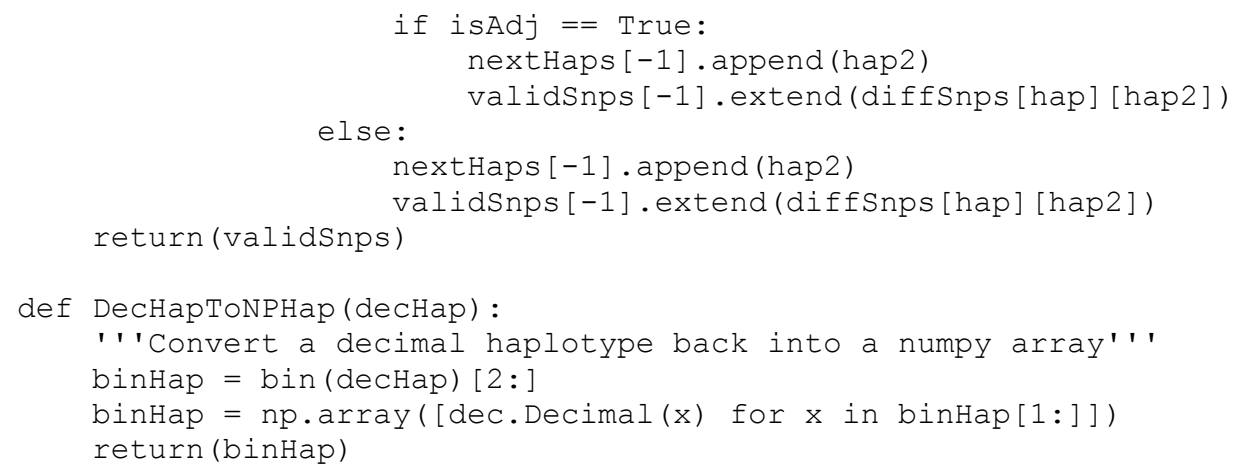




\section{Modules/IO.py}

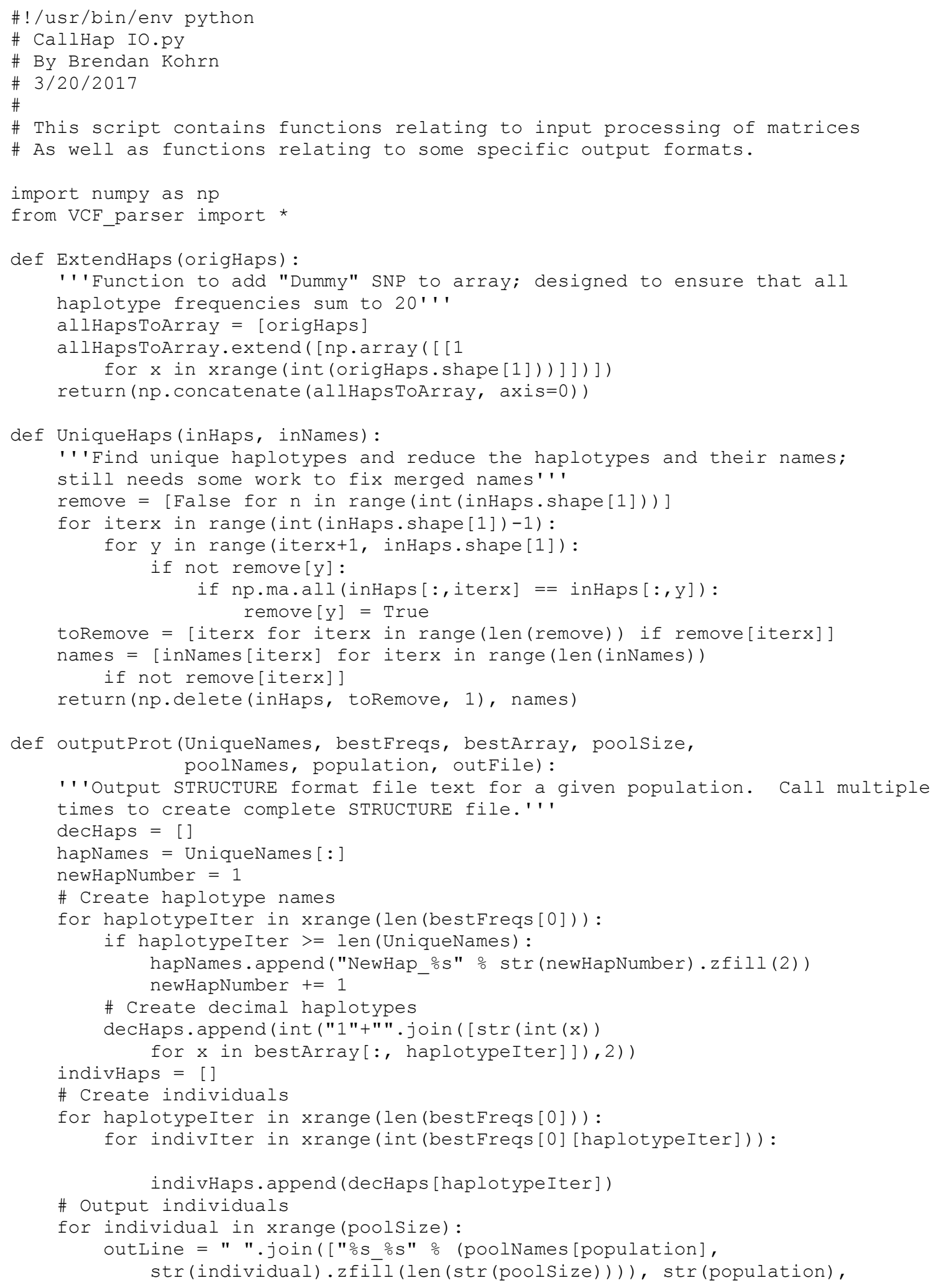




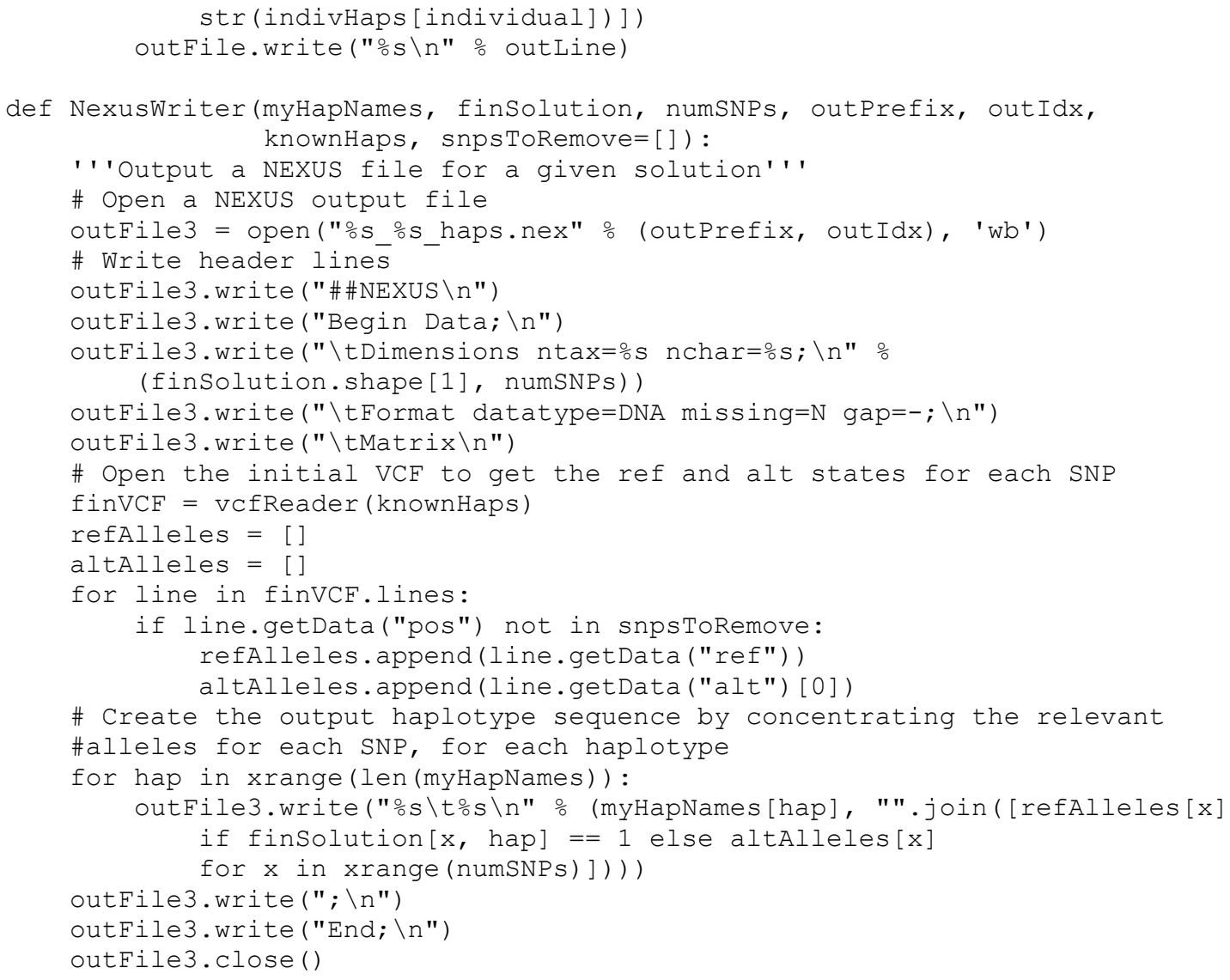




\section{Modules/VCF_parser.py}

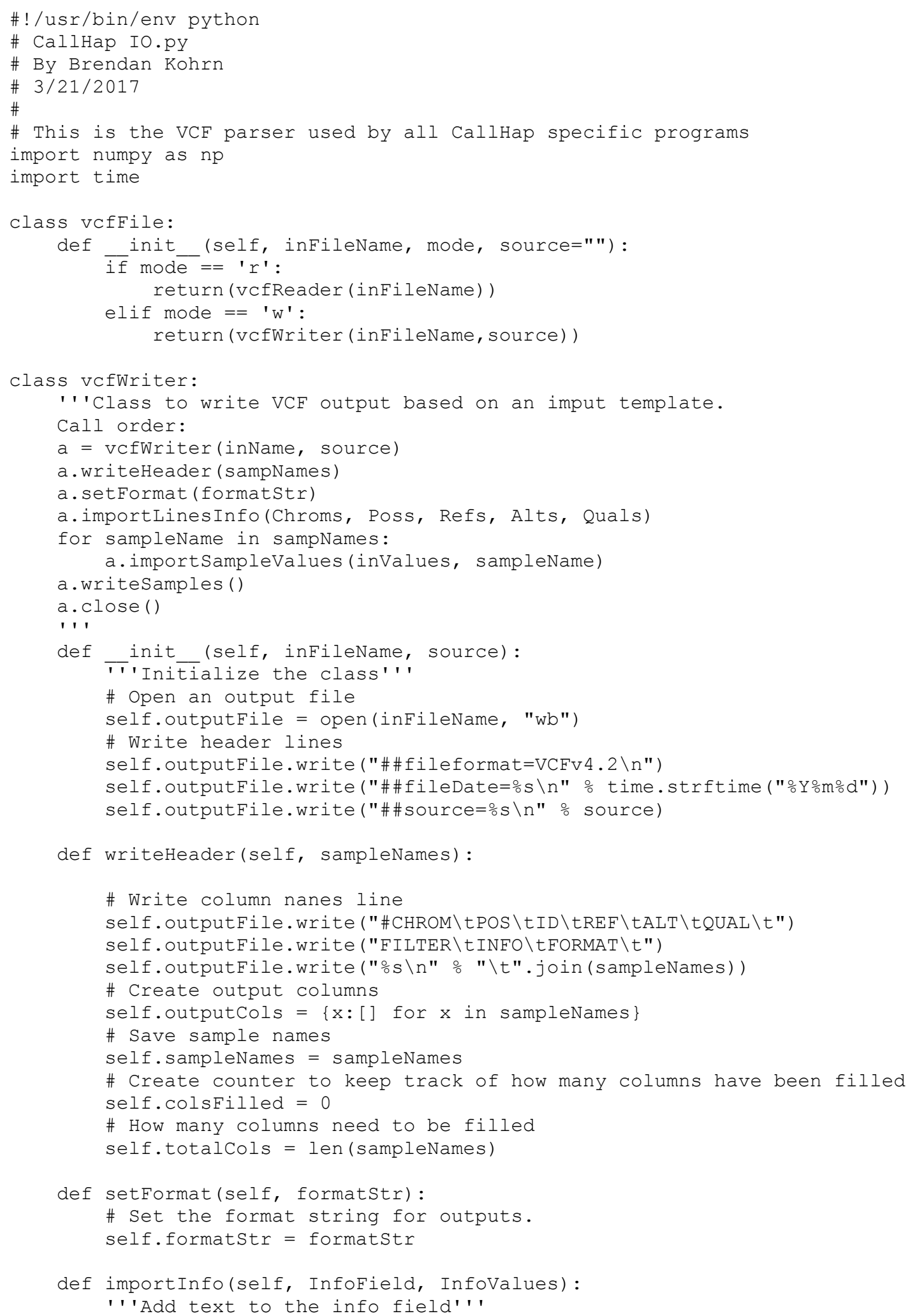




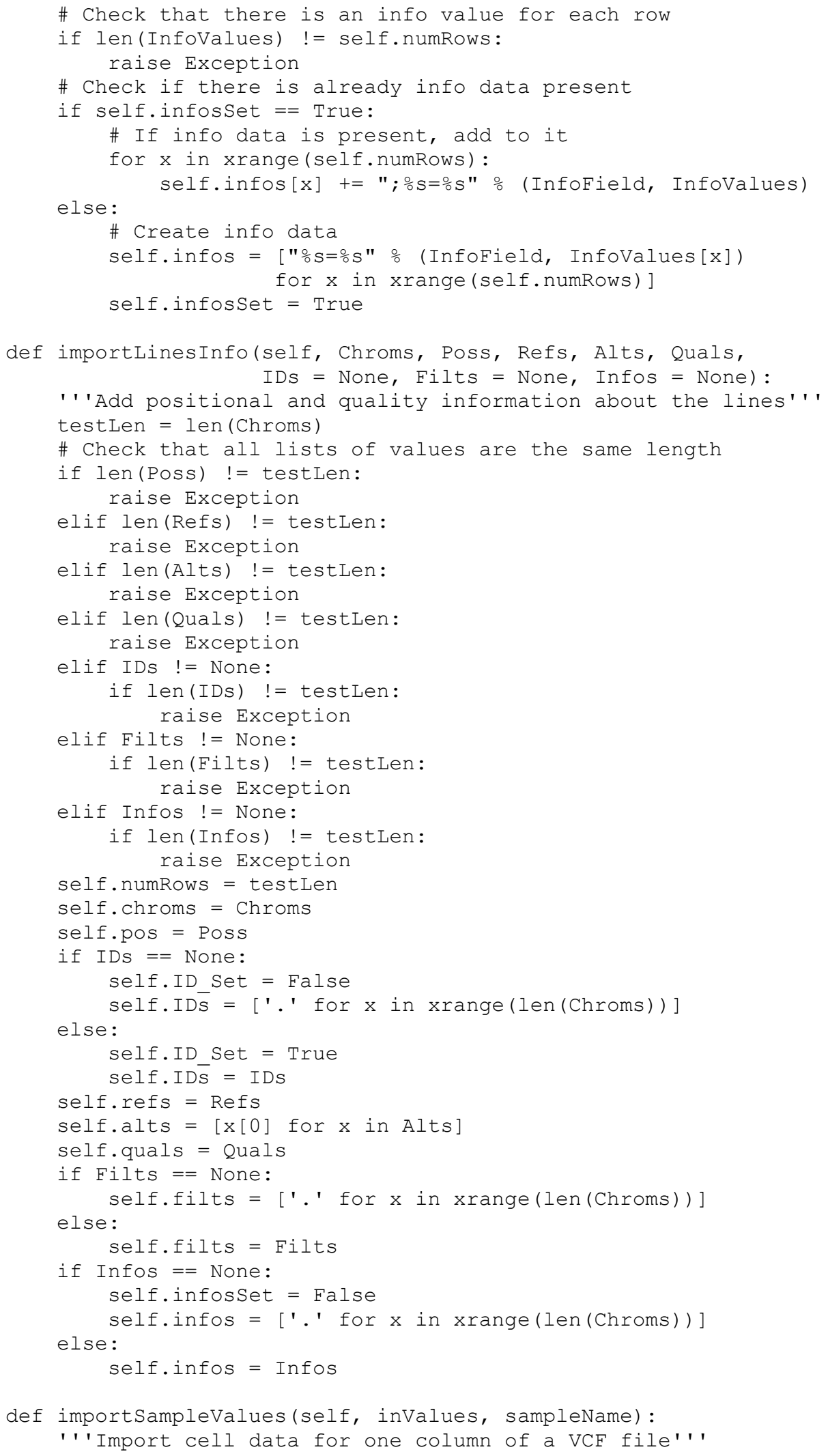




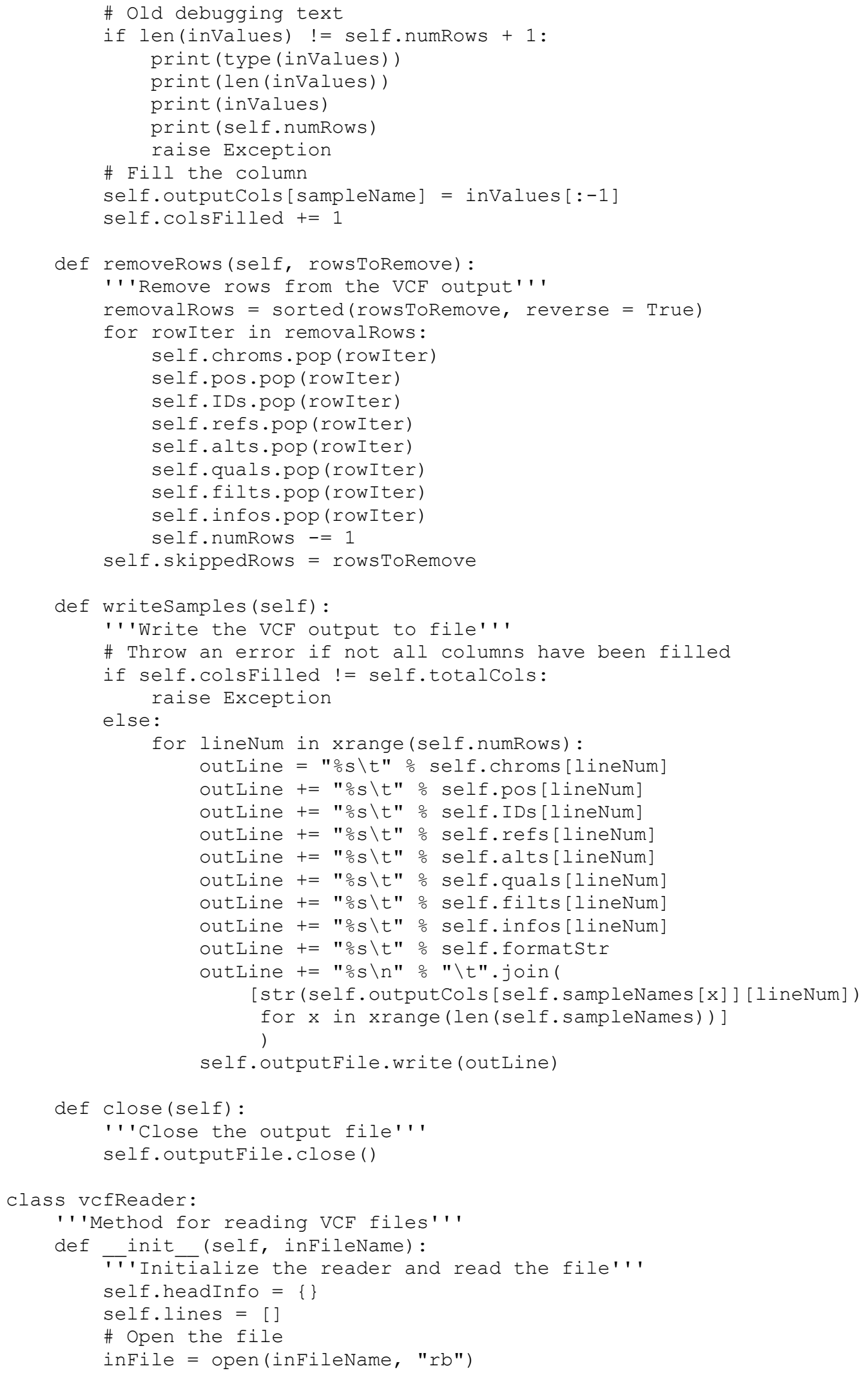




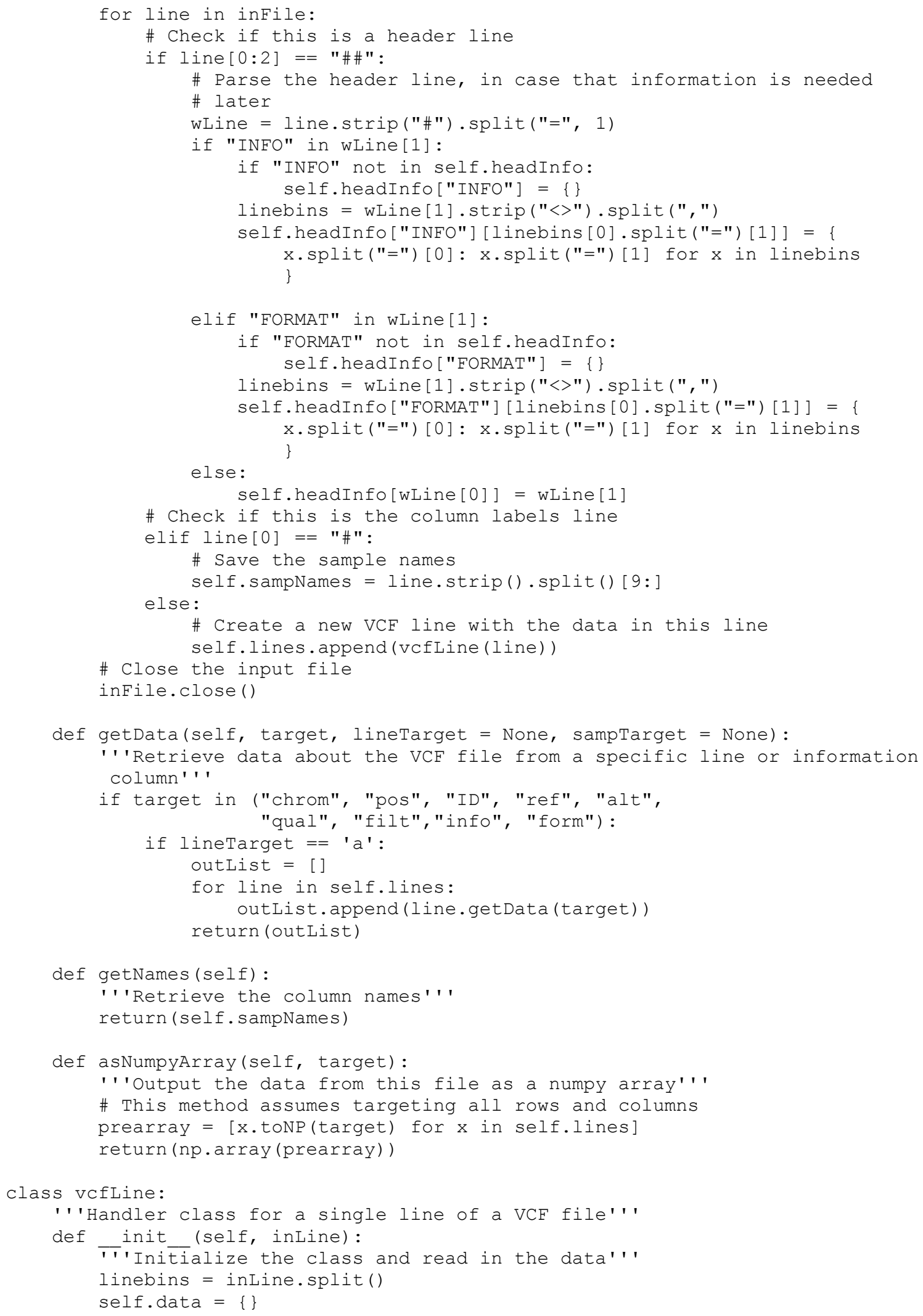




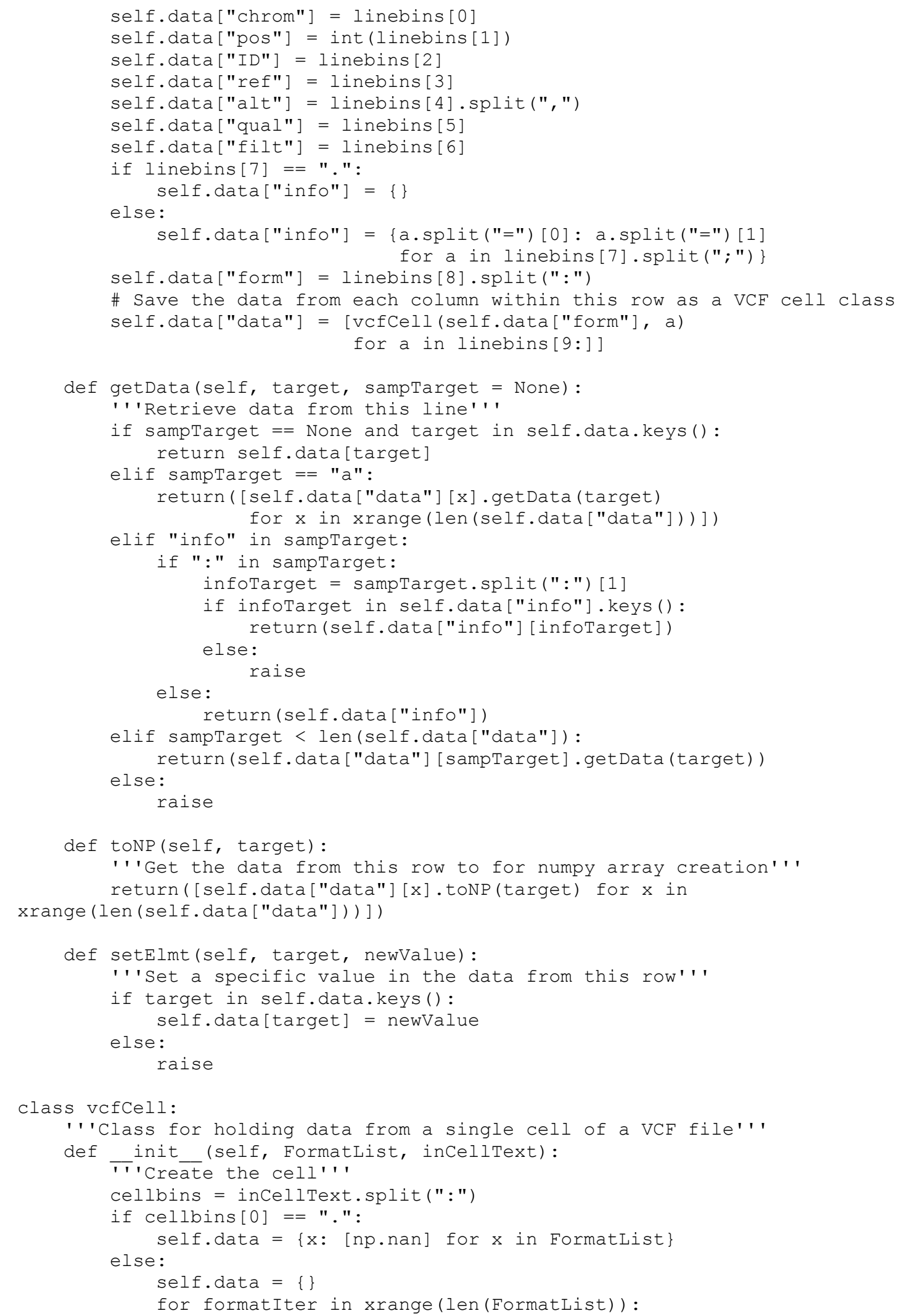




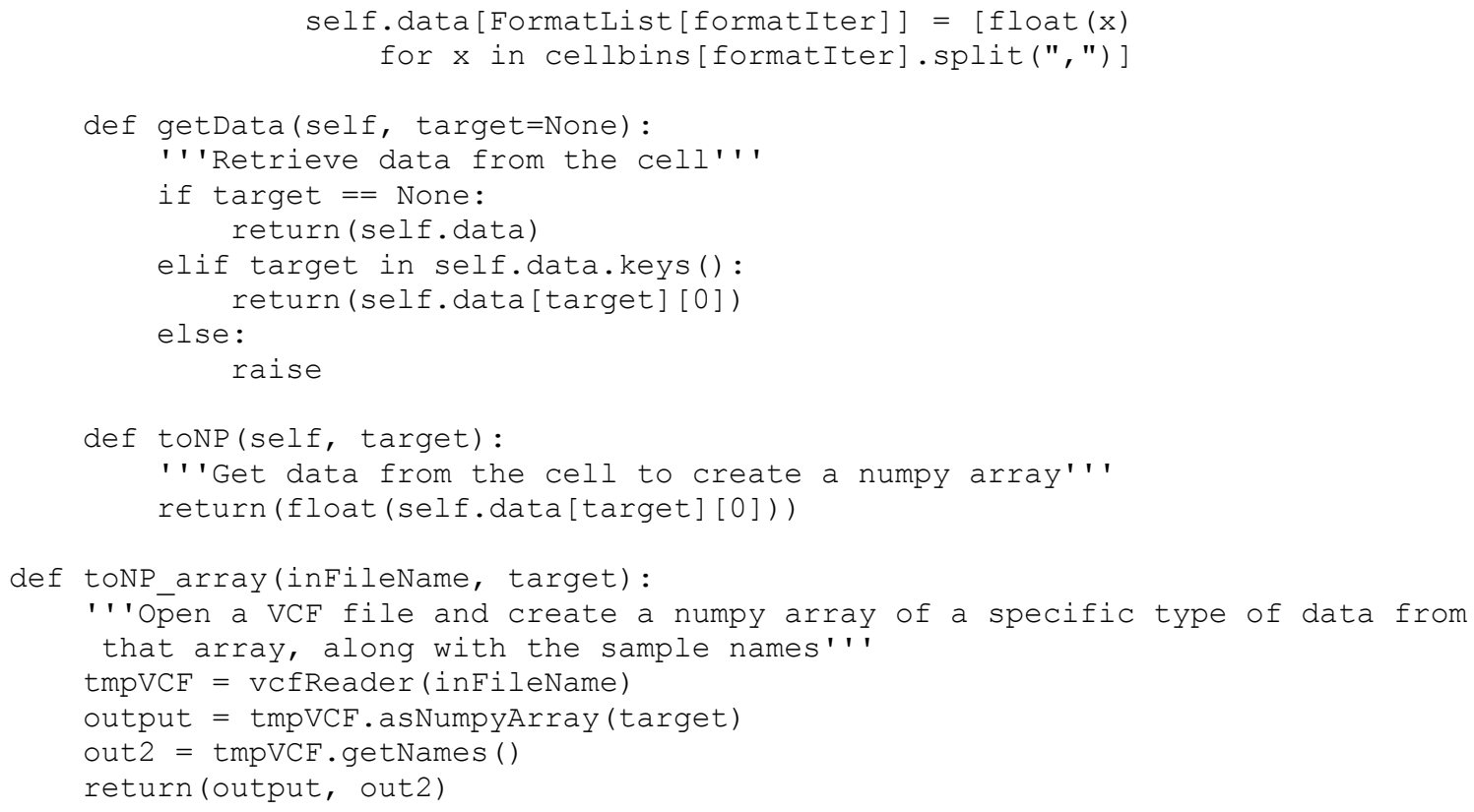




\section{Modules/parallel.py}

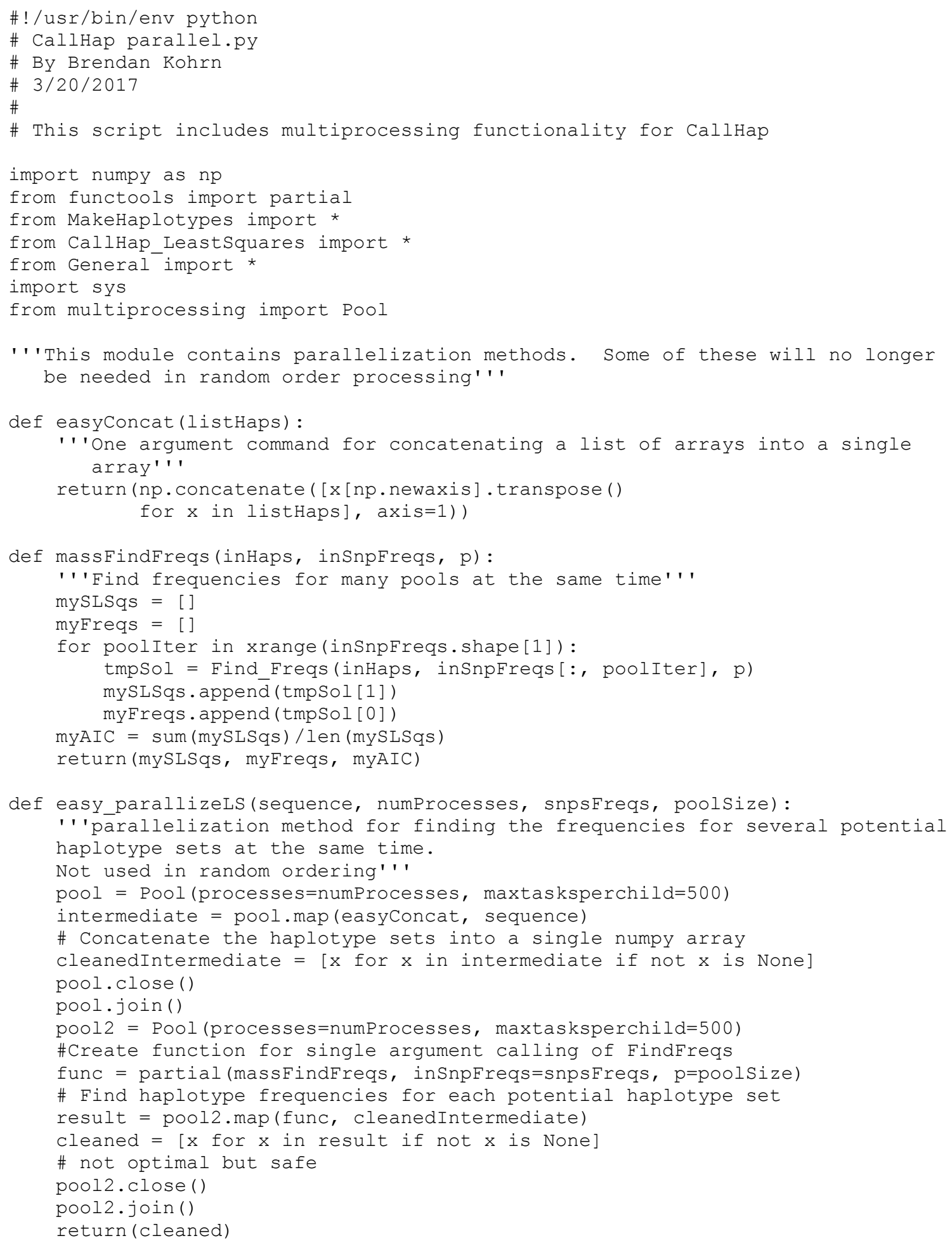

